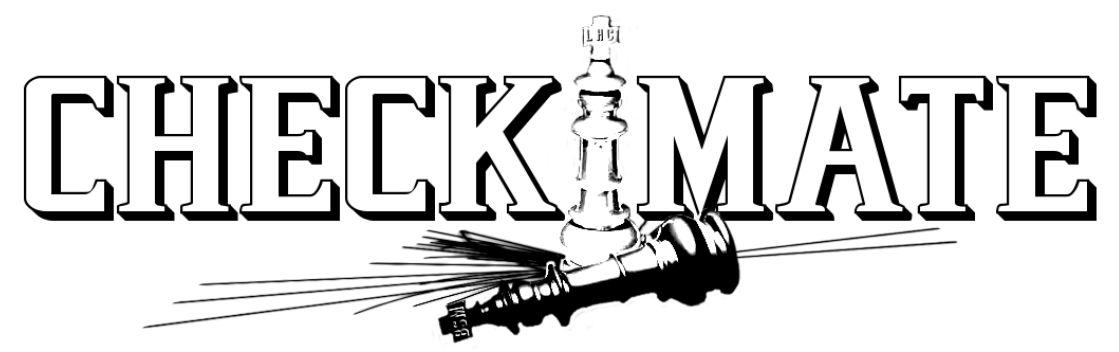

CTPU-16-36 CSIC-16-116 TTK-16-47

\title{
CheckMATE 2: From the model to the limit
}

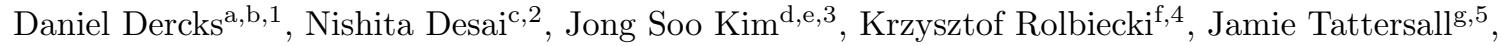 \\ Torsten Weber ${ }^{g, 6}$ \\ ${ }^{a}$ II. Institute for Theoretical Physics, University of Hamburg, Luruper Chaussee 149, D-22761 Hamburg, Germany \\ ${ }^{b}$ Bethe Center for Theoretical Physics 63 Physikalisches Institut der Universität Bonn, Nussallee 12, D-53115 Bonn, \\ Germany \\ ${ }^{c}$ Laboratoire Charles Coulomb (L2C) UMR 5221 \& Laboratoire Univers et Particules de Montpellier (LUPM) UMR 5299, \\ CNRS-Université de Montpellier, 34090 Montpellier, France \\ ${ }^{d}$ Center for Theoretical Physics of the Universe, Institute for Basic Science (IBS), Daejeon, 34051, Korea \\ ${ }^{e}$ Universidad Autónoma de Madrid, Instituto de Física Teórica, Calle Nicolás Cabrera 13-15, Cantoblanco, 28049 Madrid, \\ Spain \\ ${ }^{f}$ Faculty of Physics, University of Warsaw, Pasteura 5, 02-093 Warsaw, Poland \\ ${ }^{g}$ Institute for Theoretical Particle Physics and Cosmology, RWTH Aachen University, D-52056 Aachen, Germany
}

\begin{abstract}
We present the latest developments to the CheckMATE program that allows models of new physics to be easily tested against the recent LHC data. To achieve this goal, the core of CheckMATE now contains over 60 LHC analyses of which 12 are from the $13 \mathrm{TeV}$ run. The main new feature is that CheckMATE 2 now integrates the Monte Carlo event generation via MadGraph5_aMC@NLO and Pythia 8. This allows users to go directly from a SLHA file or UFO model to the result of whether a model is allowed or not. In addition, the integration of the event generation leads to a significant increase in the speed of the program. Many other improvements have also been made, including the possibility to now combine signal regions to give a total likelihood for a model.
\end{abstract}

Keywords: Analysis, Confidence Limits, Monte Carlo, Detector Simulation, Delphes, ROOT, LHC, Recasting, Beyond the Standard Model 12.60.-i

\footnotetext{
1daniel.dercks@desy.de

2 nishita.desai@umontpellier.fr

3 jongsoo.kim@tu-dortmund.de

${ }^{4}$ krzysztof.rolbiecki@fuw.edu.pl

5 tattersall@physik.rwth-aachen.de

6 torsten.weber@rwth-aachen.de
} 


\title{
PROGRAM SUMMARY
}

\author{
Program Title: CheckMATE \\ Journal Reference: \\ Catalogue identifier: \\ Licensing provisions: none
}

Programming language: $\mathrm{C}++$, Python

Computer: PC, Mac

Operating system: Linux, Mac OS

Keywords: Analysis, Confidence Limits, Monte Carlo, Detector Simulation, LHC, Recasting, Beyond the Standard Model

Classification: 11.9

External routines/libraries: ROOT, Python, HepMC (optional)

Subprograms used: Delphes

Nature of problem: The LHC experiments have performed a huge number of searches for new physics in the past few years. However the results can only be given for a few benchmark models out of the huge number that exist in the literature.

Solution method: CheckMATE is a program that automatically calculates limits for new physics models. The original version required the user to generate Monte Carlo events themselves before CheckMATE could be run but the new version now integrates this step. The simplest output of CheckMATE is whether the model is ruled out at 95\% CLs or not. However, more complicated statistical metrics are also available, including the combination of many signal regions.

Restrictions: Only a subset of available experimental results have been implemented.

Running time: The running time scales about linearly with the number of input events provided by the user. The event generation, detector simulation and analysis of 10000 events needs about $245 \mathrm{~s}$ for a single core calculation on an Intel(R) Xeon(R) CPU E5-2650 v2 @ 2.60 GHz with 32 GB RAM.

\section{Important Note}

- CheckMATE is built upon the tools and hard work of many people. If CheckMATE is used in your publication it is extremely important that all of the following citations are included,

- Delphes 3 [1]. https://cp3.irmp.ucl.ac.be/projects/delphes

- FastJet [2, 3]. http: //fastjet.fr/

- Anti- $k_{t}$ jet algorithm [4].

- $\mathrm{CL}_{\mathrm{S}}$ prescription [5].

- All experimental analyses that were used to set limits in the study and if the analysis was implemented by non-CheckMATE authors, the relevant implementation reference.

- MadGraph5_aMC@NLO [6] if MadGraph5_aMC@NLO is used to calculate the hard matrix element events from within CheckMATE. https://launchpad.net/mg5amcnlo

- Pythia 8.2 [7] if showering or matching done from within CheckMATE. http://home.thep.lu.se/ torbjorn/Pythia.html

- The Monte Carlo event generator that was used if .hepmc or . Ihe files were generated externally.

- In analyses that use the $m_{T 2}$ kinematical discriminant [8, 9] we use the mt2_bisect library [10]. We also include the $M_{T 2}^{b \ell}$ and $M_{T 2}^{W}$ derivatives [11]. http://particle.physics.ucdavis.edu/hefti/projects/doku.php?id=wimpmass https://sites.google.com/a/ucdavis.edu/mass/ 
- In analyses that use the $M_{C T}$ family of kinematical discriminants we use the MctLib library that includes the following variables, $M_{C T}$ [12, $M_{C T}$ corrected [13], $M_{C T}$ parallel and perpendicular 14. https://mctlib.hepforge.org/

- In analyses that use topness variable we use the topness library [15. https://github.com/michaelgraesser/topness

- In analyses that use Super-Razor [16]. 


\section{Contents}

1 Introduction 5

2 General Program Flow 6

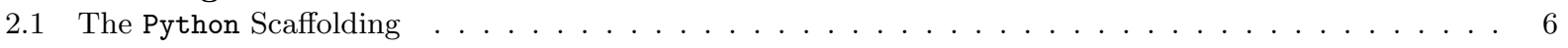

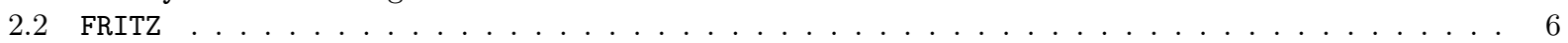

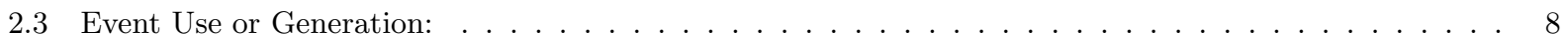

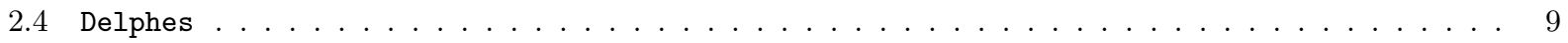

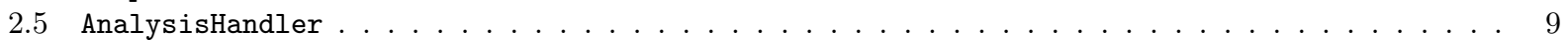

2.6 Analyses . . . . . . . . . . . . . . . . . . . . . . . . . . . . . 10

2.7 Evaluation and Output $\ldots \ldots \ldots \ldots \ldots \ldots \ldots$

3 Full List of CheckMATE Parameters

4 Example: Running CheckMATE and Understanding the Results 15

4.1 Benchmark Model . . . . . . . . . . . . . . . . . . . . . . . . . . . . . 15

4.2 Initialising and Starting CheckMATE . . . . . . . . . . . . . . . . . . . . . 16

4.3 Structure of the Results Folder . . . . . . . . . . . . . . . . . . . . . 20

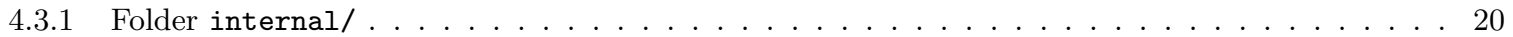

4.3 .2 Folder fritzl . . . . . . . . . . . . . . . . . . . . . . . . . . . . 21

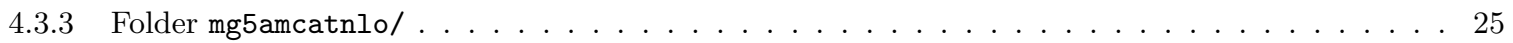

4.3 .4 Folder pythial . . . . . . . . . . . . . . . . . . . . . . . 26

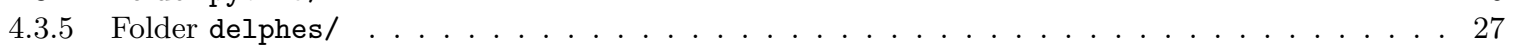

4.3 .6 Folder analysis $\ldots \ldots \ldots \ldots \ldots \ldots \ldots \ldots \ldots$

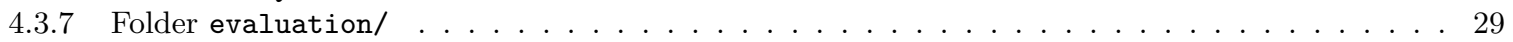

5 Available Analyses 32

6 Performance Studies $\quad 36$

7 Analysis Manager $\quad 37$

7.1 Prototyping New Analyses . . . . . . . . . . . . . . . . . . . . . . . . . . . . . . . . . . 37

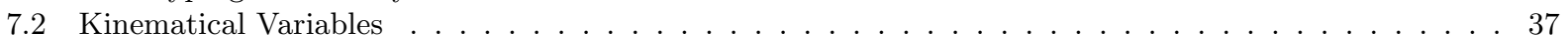

8 Summary

Appendix A Installation Instructions 39

A.1 Required Packages . . . . . . . . . . . . . . . . . . . . . . . . . . . . . 39

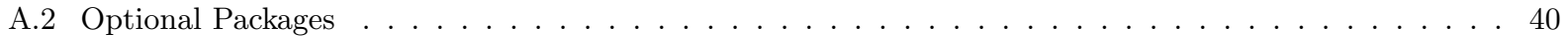

A.3 Installing CheckMATE . . . . . . . . . . . . . . . . . . . . . . . . 41

Appendix B Statistical Analysis in CheckMATE 41

B.1 1-bin Likelihood and Test Statistics . . . . . . . . . . . . . . . . . . . . . . 41

B.2 Confidence Levels and $p$-values . . . . . . . . . . . . . . . . . . . . . . . . . . . . 42

B.3 Model Independent Limits $S 95 \ldots \ldots \ldots \ldots \ldots \ldots \ldots$

B.4 Likelihood . . . . . . . . . . . . . . . . . . . . . . . . . . . 44

Appendix C Tuning 44

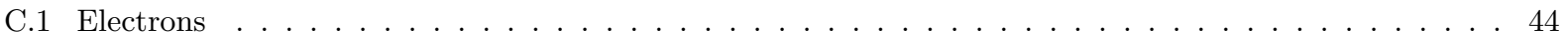

C.2 Muons . . . . . . . . . . . . . . . . . . . . . . . . . . . . 45

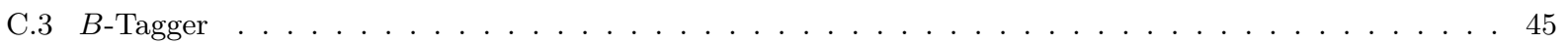

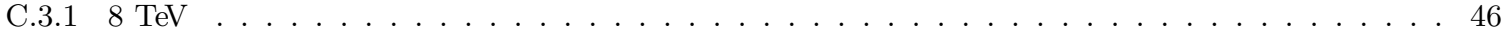

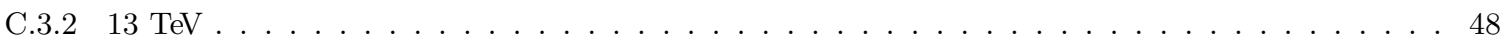




\section{Introduction}

The first years of the Large Hadron Collider (LHC) running have been a triumph with the notable discovery of the Higgs boson being a particular highlight [17, 18]. Whilst no other new states have been found yet, a large number of searches for physics beyond the Standard Model (BSM) have been performed. This dataset has profound implications for many of these BSM theories but the experimental collaborations themselves only have limited resources to investigate the many models on the market. Therefore it is imperative that theorists take up the task of testing their models against the wealth of data available.

To help with such studies, a number of tools have now been made public that allow for easy and fast model testing. One class of programs are based on the so-called 'simplified models' 19] approach. Here the tools make use of the actual LHC limits on particular topologies that are commonly seen in BSM. The limits are then adjusted to account for the correct branching ratios in the actual model under test. The advantage of such an approach is that these programs are very quick to return an answer. However the big disadvantage is that if the new physics model contains final state topologies not originally tested, the limit will be severely weakened compared to the true result. Unfortunately this is very common in realistic models that may have longer or asymmetric decay chains. Examples of tools that use simplified models are SModels [20, 21] and FastLim 22] for supersymmetry and XQCUT [23] for models with extended quark sectors. Futhermore, if limits from a simplified model analysis are used for a different model (e.g. one predicting different angular correlations or extra intermediate particles) the results may not be accurate. This severely limits the applicability to classes of models with particles of exactly the same spin and an identical decay chain to the one originally tested.

The second approach 7 for theorists to test models against the LHC data is to essentially follow the same procedure that the experimental collaborations perform themselves, or to "recast" 8 Here, Monte Carlo (MC) events are simulated for the particular model under test and these events are then passed through a detector simulation that returns reconstructed final state objects. The same experimental cuts used by the experiment are then placed on these objects and limits are placed on the number of events seen in predefined signal regions. This chain is validated against benchmark models provided by the experiments. Once validated, the recast analysis can be used to test any model by changing the MC events supplied. Whilst this method has the disadvantage of being slower than the simplified model approach, the big advantage is the generality that allows for a large range of different theories to be tested. Examples of tools that use recast analyses are CheckMATE [26, 27] and MadAnalysis [28, 29] while the newly released Contur [30] uses the RIVET [31] library of Standard Model measurements.

So far all the recast based tools require the user to provide externally generated events that have been showered, hadronised and already have any required underlying event modelling. This has severe performance drawbacks aside from the obvious extra effort for the end user to generate these events. Firstly, the Monte Carlo events must be stored to disk and this process involves writing and re-reading several 10's or even 100's GB for the required statistics ${ }^{9}$ Consequently, even when we are just testing a single model point the Monte Carlo events already require significant amounts of free space. However, a greater issue is if we want to test many model points on a large cluster. In a cluster architechture where many jobs run from a single hard drive, the reading and writing of events is already the limiting factor for just $2-3$ simultaneous processes.

To solve this problem, we present CheckMATE 2 which integrates both MadGraph5_aMC@NLO and Pythia 8 into a complete model testing loop. As a consequence, the user only has to provide an SLHA file [32, 33. in the case of supersymmetry or a UFO [34] model file that can be produced by a variety of tools such as FeynRules [35, 36, SARAH [37, 38, or LanHEP 39] for other models and the event generation is then taken care of internally by CheckMATE. We also note that the improved efficiency due to skipping the event

\footnotetext{
${ }^{7}$ Another approach, that we do not discuss here, is training a machine learning algorithm on already tested models which has been pioneered by SUSY-AI [24].

8 "To Recast" is a a verb coined from Ref. 25] and is now increasingly used as shorthand to describe the full process of reproducing an experimental analysis.

${ }^{9}$ We find e.g. that 10,000 hadronised events require between $\sim 1$ and $\sim 10 \mathrm{~GB}$.
} 
file storage results in a single process running up to $\sim 40 \%$ faster. More importantly however, is the far improved cluster processing. That means running many simultaneous jobs from a single hard disk is now made possible without any reduction in performance.

The incorporation of the event generation is not the only improvement in CheckMATE 2 though. Over 60 experimental analyses from the LHC collaborations are now available covering the 7, 8 and $13 \mathrm{TeV}$ runs and more are continually being added. In addition, CheckMATE 2 now contains 14 TeV high luminosity LHC analyses as well. Consequently the user can now go beyond simply setting limits on models with the current data and investigate what is the ultimate LHC reach to the model of interest. Further improvements have also been made to the AnalysisManager introduced in Ref. 27. so that users can more easily investigate new ideas for LHC searches. These include more LHC kinematical observables being included and additional tools that make the statistical analysis of the results easier.

We begin this paper in Section 2 by giving an overview of the individual building blocks that make up the CheckMATE program. We follow in Section 3 by giving the full list of available CheckMATE parameters that may be useful to more advanced users. To more easily explain how CheckMATE is used, we then provide an example run in Section 4 that also discusses in more detail some of the most commonly used options. A brief summary of the currently available analyses is provided in Section 5 . Section 6 investigates the performance improvements of the new version of CheckMATE over the old in more detail while Section 7 explains the improvements to the AnalysisManager. Finally, we summarise in Section 8 . Appendix A gives installation instructions, Appendix $\mathrm{B}$ covers the statistical methods used in CheckMATE in more detail and Appendix C describes the updated lepton identification performance and $b$-tagging efficiencies now used in CheckMATE.

\section{General Program Flow}

CheckMATE incorporates many individual modules which cover the steps necessary for model testing. A flowchart illustrating the modules and the data passed internally between them is given in Figure 1. The modules are embedded in a Python scaffolding which handles the user prompts, the file I/O and the setup of the core modules which we describe in more detail below.

\subsection{The Python Scaffolding}

The initialisation is performed by a CheckMATE Python script which reads in user input (either via a file or command line), and writes the configuration files required by the FRITZ and AnalysisHandler modules to set up event generation, showering and detector simulation followed by recasting of the experimental analysis. The computation-heavy parts are taken over by the individual modules coded in C++ described below, which call as required external libraries or programs (viz. MadGraph5_aMC@NLO, Pythia 8, HepMC, Delphes and ROOT). After the recast analyses are run, the results are again collected and processed by the Python script to check against published 95\% confidence level (CL) upper limits. The ratio of expected signal to the $95 \%$ CL upper limit and whether the point is still allowed is reported as the default primary result. Further options for statistical evaluation are discussed in Section 2.7

\subsection{FRITZ}

FRIT2 10 (Flexible Rapid Interactive Tool Zipper) denotes the core C++ program of CheckMATE. Depending on the provided data and settings, it connects to and runs MadGraph5_aMC@NLO, Pythia 8 and Delphes, followed by the AnalysisHandler and all the analyses requested by the user. Except for the LHE files produced by MadGraph5_aMC@NLO, intermediate data, e.g. the simulated Monte Carlo events generated by Pythia and/or the detector level objects produced by Delphes, are passed on-the-fly between the individual modules. This is a great improvement on the original CheckMATE version 1 in which the generated events as well as the detector level objects had to be stored and then re-read from hard disk. Given that a typical

\footnotetext{
${ }^{10}$ The name Fritz is derived from a German chess program of the same name, see Ref. 40, 41, and the very first chess computer program one of the authors (DD) played.
} 


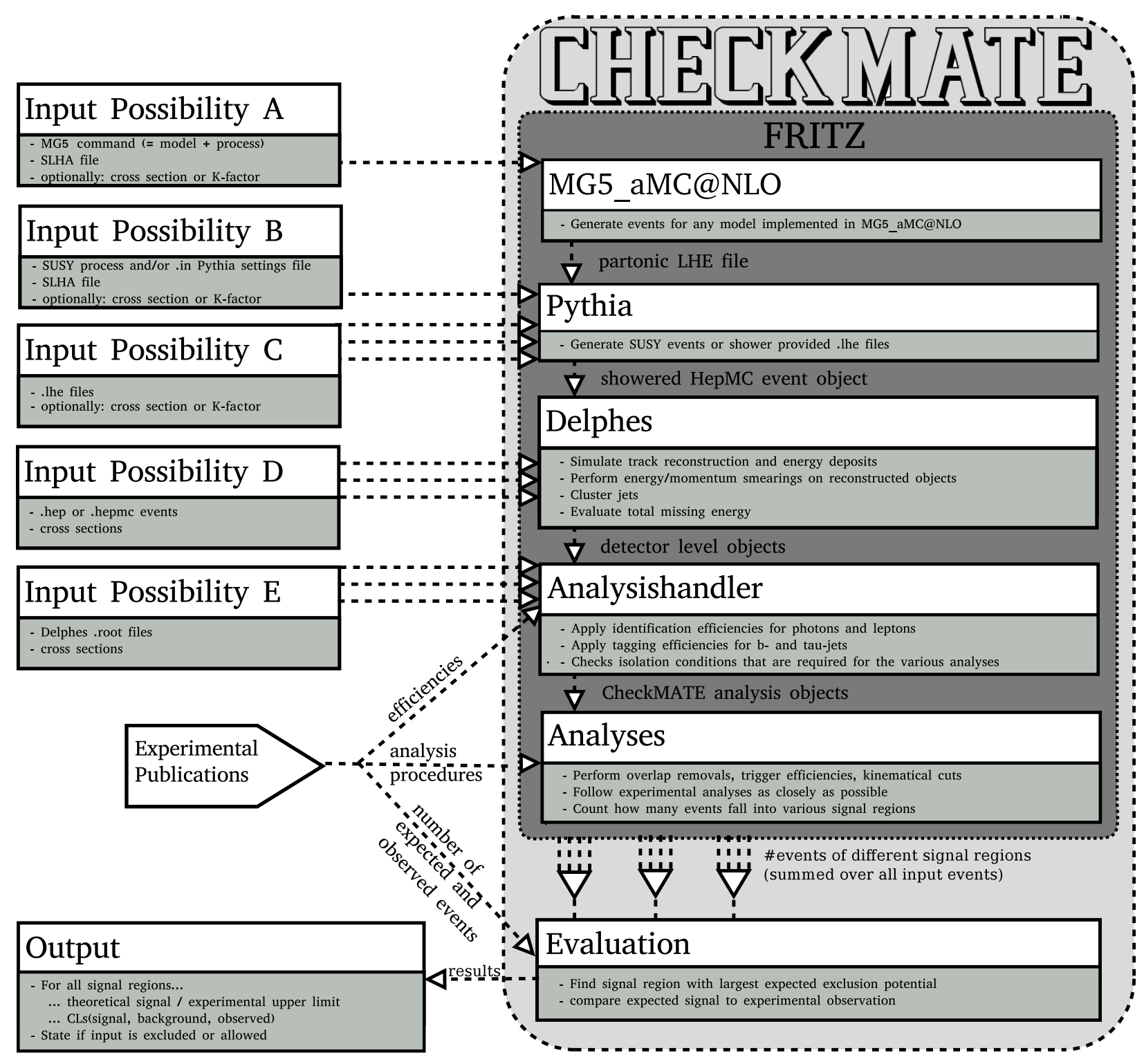

Figure 1: Flow chart to demonstrate the chain of data processing within CheckMATE. 
BSM Monte Carlo event file including hadronised final states and a sufficiently high statistical sample easily reaches file sizes of several GB, significant time is saved in I/O by this improvement ${ }^{11}$ besides removing the requirement for large storage. However, if the user requires so, the intermediate objects can be written to the disk using the switches WritePythiaEvents and WriteDelphesEvents, cf. Section 3.

\subsection{Event Use or Generation:}

One of the core parts of Monte Carlo based collider phenomenology is the simulation of final state configurations that would be produced in a collider experiment if a particular model of BSM physics was true. In the first version of CheckMATE, the event generation had to be done externally by the user. MC event files and the corresponding cross section - either from the same event generator or from an external cross section calculator like Prospino [42,46] or NLLFast [42, 43, 47,51] - were mandatory input parameters which were then processed via Delphes within CheckMATE. Besides the practical inconvenience that every CheckMATE user had to use an external event generator, the forced split between event generation and detector simulation/analysis also yields a computational disadvantage as already explained above. Consequently, the new CheckMATE version now provides an automatic link to both MadGraph5_aMC@NLO [6] and the Pythia 8 [7] event generation. With this new functionality, CheckMATE provides different types of modes to either run MadGraph5_aMC@NLO and Pythia 8 or simply use already generated event files:

Provide an externally produced .hepmc or .hep event file: We first emphasise that if the user wishes to provide Monte Carlo events in either .hepmc or .hep format to CheckMATE, this option is still supported. CheckMATE will then pass these events directly to Delphes for detector simulation.

Generate events entirely using Pythia 8: Pythia 8 is capable of generating events for BSM models followed by parton showering and hadronisation of the final state. This functionality can be accessed by CheckMATE in two different ways.

The first possibility is to provide the Pythia 8 setup via an .in file which uses the Pythia 8 internal syntax, see refs. [7, 52, 53], to set the internal parameters. This mode allows for the full flexibility of the Pythia 8 program as all parameters can be changed via this input file method. Most importantly, the . in file is used to define the model and the list of processes which should be generated. All model parameters (e.g. couplings, masses, widths, branching ratios etc.) must be provided in the input file. If a supersymmetric (SUSY) process is desired, the SLHA file [32, 33] which specifies the parameter point must be provided within the input file in the standard Pythia 8 syntax.

Given the popularity of SUSY models, we provide an additional shortcut to generate SUSY processes without the need to provide a full input card. All showering and hadronisation parameters will then be taken from the default Pythia settings. The process can be set directly using a simplified MadGraph5_aMC@NLO-like syntax e.g. Pythia8Process: p p > go go to initiate gluino pair production. A further shorthand to refer to classes of SUSY particles is also available. The full list of available processes using this running mode is given in Section 3 .

In both cases, CheckMATE will use Pythia 8 to simulate the given processes and will directly perform detector simulation followed by applying analysis routines. As explained above the events are not stored unless explicitly demanded by the user.

By default, CheckMATE uses the cross section and statistical uncertainty provided by Pythia 8. A user can also provide a cross section or a $K$-factor, i.e. $\sigma_{\mathrm{NLO}} / \sigma_{\mathrm{LO}}$, calculated via an external code (see Section 3).

Generate events with MadGraph5_aMC@NL0, shower and hadronise with Pythia 8: This option allows a user to both generate the hard matrix element using MadGraph5_aMC@NLO by providing a process card (and parameter and run cards if necessary) followed by showering and hadronisation of the resultant

\footnotetext{
${ }^{11}$ We show later that we can gain a factor of 3 in speed between CheckMATE version 1 and CheckMATE version 2 depending on the details of the benchmark model and the number of parallel runs. Details can be found in Section 6 .
} 
LHE files via Pythia. This allows users to generate Monte Carlo events for a huge range of BSM models using the UFO file format [34].

Shower and hadronise externally provided . Ihe files: If a user wishes to calculate the matrix element and generate events with a different generator than MadGraph5_aMC@NLO, e.g. WHIZARD [54, 55] or CalcHep [56, this is also possible as long as the program is able to output event files in the . The format. The advantage of this approach is that the hard process simulation and the parton showering and hadronisation steps can be performed rather independently and it is usually the latter two which take the most computational effort and require most disk space. CheckMATE can take lightweight . Ihe files produced by an external tool and use Pythia 8 to shower/hadronise on-the-fly as described above. A default . in file is then used to set the collision energy and to retain default Pythia 8 settings but if necessary users can also provide their own file instead (if e.g. a user wishes to turn off multi-particle interactions etc.)

Pythia 8 internally generates random numbers starting from a default seed. The user can further provide the random number seed in two ways - first by setting an integer seed via RandomSeed: $\mathrm{X}$ and second by providing a binary state for the Pythia 8 run using the Pythia8Rndm key in the CheckMATE input file. Each Pythia 8 run writes out the random state at the beginning and end of the run in the files rndm-init.dat and rndm-end.dat respectively. These can then be used either to reproduce a run or while adding events to ensure there is statistical independence of the two runs.

\subsection{Delphes}

The events that were either generated internally or provided by the user are then passed to the detector simulation program Delphes [1. In CheckMATE 2.0, contrary to the original CheckMATE described in Ref. 26], Delphes is now only used to simulate the calorimeter and tracking using the standard detector settings for ATLAS and CMS along with jet reconstruction. The identification efficiencies for final state particles are now performed internally by CheckMATE. In addition, the output event object is extended to include generator level particles which are required for external $b$ - and $\tau$-tagging.

The results of the detector simulation are passed as ROOT objects and are typically not saved on disk (unless explicitly requested) but instead immediately processed by the analysis framework described below. By setting WriteDelphesEvents to True (see also Section 3), a .root output file can optionally be created and used as an event file in a future CheckMATE run, see also Figure 1

If a user decides to test only ATLAS or only CMS analyses, Delphes needs to run only once per event. Otherwise, each input event is processed by two independent Delphes runs and independent detector level objects for ATLAS and CMS analyses are respectively created.

\subsection{AnalysisHandler}

The detector level objects created in the previous step contain reconstructed electrons, muons, photons, jets, tracks, clustered calorimeter cells and the missing transverse momentum vector. These are now further processed by a so-called AnalysisHandler before being passed to the actual data analysis codes.

Depending on the list of analyses selected by a user, final state objects are tested against a list of isolation, identification and tagging conditions which set individual tags on those candidates which pass the respective constraints. For that purpose, CheckMATE first determines which constraints have to be considered in order to provide all analyses with the required information on the final state objects. As an example, let us assume a user chose three analyses out of which two require tight leptons (usually this means electrons or muons at the LHC) and the third one vetoes events with medium leptons. Then the AnalysisHandler using a lepton list passed from Delphes will create three new lepton lists, corresponding to different identification working points: tight $\subset$ medium $\subset$ loose. The decision about the assignment for a particular lepton is based on its momentum and takes into account identification efficiencies reported by the experiments, see 
also Appendix C. Finally, isolation criteria will be checked for each of the leptons. The AnalysisHandler will also apply simplified $b$ - and $\tau$-tagging algorithms, if required 12

CheckMATE uses a set of independent AnalysisHandlers with individual tagging efficiencies depending on the list of analyses chosen by the user. Due to the evolution of the reconstruction and identification algorithms, there exist separate AnalysisHandlers for analyses performed on 7,8 and $13 \mathrm{TeV}$ data and for projective studies at $14 \mathrm{TeV}$ centre-of-mass energy. For each of those there is an independent ATLAS and CMS version. The tunings for 7 and $8 \mathrm{TeV}$ are described in more detail in Ref. 26] while the updates for $13 \mathrm{TeV}$ are given in Appendix C.

\subsection{Analyses}

After all detector level objects have been properly prepared by the AnalysisHandler(s), these are processed event by event by each analysis selected by a user when starting CheckMATE. They are internally coded in a framework that allows for an easy extension to new upcoming experimental results and allows users to easily update given analyses or implement their own, see also the original CheckMATE publication in Ref. 26. For a detailed description of the AnalysisManager framework see 27.

Each event is processed by checking isolation criteria, removing overlapping objects and implementing the cuts that define the signal regions. The analysis program then determines how many events in total satisfy certain signal region criteria and stores this information in a human-readable output for each separate input event file or alternatively each separate Pythia run. In addition we also store the actual number of Monte Carlo events (i.e. the efficiency times acceptance $\mathcal{A} \times \epsilon$ ) and the number of signal events $S=\mathcal{L}_{\text {int }} \times \sigma \times \mathcal{A} \times \epsilon$, where $\mathcal{L}_{\text {int }}$ is the integrated luminosity, and the cross section $\sigma$ is provided either internally by Pythia 8 or externally by the user. In case of weighted events, the event weights are taken into account while calculating the efficiency.

\subsection{Evaluation and Output}

The final step of the program consists of a statistical evaluation of the results. For each individual signal region of every chosen analyses, the total number of expected signal events $S$ is determined by summing up the results from each input event file (or event generation run) as explained later in Section 4.3.7. The total $1 \sigma$ uncertainty $\Delta S$ on this number is determined from both the statistical uncertainty, given by the number of Monte Carlo events, and the systematic uncertainty, which is estimated from the total uncertainty on the signal cross section given by the user as an optional parameter. These numbers are compared to the results published in the respective experimental search. There are two possible ways to perform the comparison (see also Ref. [26]): in the standard approach, the number $S$ is tested against a pre-calculated model independent $95 \%$ CL limit S95. Alternatively, a user can choose to calculate the proper $\mathrm{CL}_{\mathrm{S}}$ value by folding in the uncertainty on the model prediction. This is not only more accurate but allows for testing against limits other than 95 \% CL. In both cases, CheckMATE calculates the observed $\mathrm{CL}_{\mathrm{S}}$ value using the actual data recorded and the expected $\mathrm{CL}_{\mathrm{S}}$ value which assumes the observed data equals the background expectation. In contrast to the earlier CheckMATE version, the current version uses its own routines to perform the statistical calculations using the algorithm explained in Appendix B. Besides those two means of model testing, CheckMATE optionally allows for the calculation of the likelihood of the final result. This allows for model parameter fits and corresponding confidence limit evaluations. The calculation of the likelihood is explained in detail in Appendix B

The results of the evaluation are then output for every signal region of every analysis. If multiple signal regions are considered, the model point is determined to be "excluded" or "allowed" based on the signal region which has the best sensitivity assuming background-only hypothesis evaluated by the experiment. This is done to avoid erroneously ruling out a model point due a downward fluctuation of the observed events in a signal region that is not expected to be sensitive in the first place.

\footnotetext{
${ }^{12}$ In the first CheckMATE version, all these steps have been performed within the Delphes framework by generating a runspecific Delphes detector card. That made it impossible to re-use Delphes output files, either within CheckMATE to a posteriori test additional analyses or outside CheckMATE with a different Delphes-based analysis framework like e.g. MadAnalysis5 28]. Therefore, the CheckMATE and MadAnalysis5 teams mutually agreed to switch to a final state post-processing outside Delphes, see also [57.
} 


\section{Full List of CheckMATE Parameters}

There exist many optional parameters within CheckMATE which can change the standard behaviour of the code and here we describe their usage. These parameters can either be provided via the input file or alternatively, CheckMATE can be set up directly within the command line by adding -parameter value pairs after the ./CheckMATE command. The second alternative is unfortunately only possible for a setup with a single process as only one - $\mathrm{p}$ command can be provided. If more than one process needs to be analysed in the same run, one either has to use the input file or use the add feature described below.

Analyses: The full list of currently implemented analyses is given in Section 5 . The following examples show how to specify which of these CheckMATE should take into account using an input file (command line),

- Analyses: atlas_1404_2500 (-a atlas_1404_2500) tests only the analysis atlas_1404_2500.

- Analyses: 8TeV (-a 8TeV) tests all implemented analyses which correspond to $\sqrt{s}=8 \mathrm{TeV}$. Alternative values are $7 \mathrm{TeV}, 13 \mathrm{TeV}$ and $14 \mathrm{TeV}$.

- Analyses: atlas8TeV (-a atlas8TeV) tests all implemented ATLAS analyses of the given centre-of-mass energy. Similarly for cms.

The above specifiers can be combined via a simple separation with commas,

e.g. - a cms8TeV, atlas_1404_2500 tests all $8 \mathrm{TeV}$ CMS analyses and the single ATLAS analysis atlas_1404_2500. All analyses combined this way must correspond to the same center-of-mass energy, otherwise CheckMATE will abort.

Invisible PIDs: Physics beyond the Standard Model which addresses the dark matter problem often predicts the existence of one or more stable, light particles that only interact weakly with ordinary matter. Whilst Delphes automatically identifies all neutral particles in the Minimal Supersymmetric Standard Model (MSSM), other BSM particles have to be explicitly declared as invisible as they are otherwise considered as exotic hadrons which deposit their energy in the hadronic calorimeter. As an example, a Higgs portal model with a stable scalar would require placing in the parameter file the following setting: Invisible PIDs: 35.

Result file columns: CheckMATE stores the results of its analyses in various files to allow for a detailed investigation how the final result was determined. The standard content of these result files - we describe them in more detail in section 4 - is adaptable such that more intermediate results may be stored. By setting the corresponding ResultFileColumns parameter to $a, b, c, \ldots$ the corresponding file(s) are set to contain respective information $a, b, c$. EventResultFileColumns and ProcessResultFileColumns can be taken out of the following set:

analysis, sr, totalmcevents, totalnormevents, totalsumofweights, totalsumof weights2, signalsumofweights, signalsumofweights2, signalnormevents, signal_err_stat, signal_err_sys, signal_err_tot.

The names are mostly self-explanatory; the prefix total- refers to the full input sample whereas signal- corresponds to the subset of events which pass the respective signal region cuts. sumof weights2 corresponds to sum of squared weights which is an important quantity to calculate the statistical uncertainty properly in case of weighted events. normevents correspond to the physical number of events after normalising to the provided cross section and the analysis' respective integrated luminosity.

TotalResultFileColumns and BestSignalRegionResultFileColumns can in addition use the following columns:

obs, bkg, bkgerr, eff, eff_err_stat, eff_err_sys, eff_err_tot, s95obs, s95exp, robs, robscons, robsconssysonly, rexp, rexpcons, rexpconssysonly, clsobs, clsobs_err, clsexp, clsexp_err, likelihood, 


\begin{tabular}{|c|c|c|}
\hline Parameter card & Terminal & Description \\
\hline \multicolumn{3}{|c|}{ General options } \\
\hline Name: $\mathrm{X}$ & $-\mathrm{n} X$ & Gives name $\mathrm{X}$ to the run and specifies out \\
\hline Analyses: $\mathrm{X}$ & $-\mathrm{a} X$ & $\begin{array}{l}\text { States which analysis/es X should be applied to the event } \\
\text { files; see the text for more details. }\end{array}$ \\
\hline SLHAFile: $\quad \mathrm{X}$ & -slha X & $\begin{array}{l}\text { Use SLHA file X. Mandatory if event generation using } \\
\text { Pythia } 8 \text { is requested. }\end{array}$ \\
\hline InvisiblePIDs: $\quad \mathrm{X}$ & -invpids X & $\begin{array}{l}\text { BSM Monte Carlo Particle IDs [58] which are invisible for } \\
\text { the detector; see the text. }\end{array}$ \\
\hline QuietMode: True & $-\mathrm{q}$ & No terminal output is produced. Automatically sets -sp. \\
\hline SkipParamCheck: True & $-s p$ & Skips startup parameter check. \\
\hline SkipAnalysis: True & $-\mathrm{sa}$ & Skips analysis step. Requires -wp8 or -wd. \\
\hline SkipPythia: True & -spy & $\begin{array}{l}\text { Only if . The files are provided. These are not showered } \\
\text { by Pythia } 8 \text { but instead directly processed via Delphes.* }\end{array}$ \\
\hline SkipEvaluation: & $-\mathrm{se}$ & Skips evaluation step. \\
\hline RandomSeed: $\mathrm{X}$ & $-r s \mathrm{X}$ & $\begin{array}{l}\text { Chooses fixed seed } \mathrm{X} \text { for the random number generator to } \\
\text { render output deterministic. }\end{array}$ \\
\hline \multicolumn{3}{|c|}{ Options related to output } \\
\hline WritePythiaEvents: True & -wp8 & Writes .hepmc files produced by Pythia 8 on disk. \\
\hline WriteDelphesEvents: True & $-w d$ & Writes .root files produced by Delphes on disk. \\
\hline $\begin{array}{l}\text { EventResult- } \\
\text { FileColumns: } \quad \mathrm{X}\end{array}$ & $-\operatorname{erfc} X$ & $\begin{array}{l}\text { Sets columns which are stored in event-wise result files; } \\
\text { see the text for more details. }\end{array}$ \\
\hline $\begin{array}{l}\text { ProcessResult- } \\
\text { FileColumns: } \mathrm{X}\end{array}$ & $-\operatorname{prfc} X$ & $\begin{array}{l}\text { Sets columns which are stored in process-wise result files; } \\
\text { see the text for more details. }\end{array}$ \\
\hline $\begin{array}{l}\text { TotalResult- } \\
\text { FileColumns: } \quad \mathrm{X}\end{array}$ & -tefc $x$ & $\begin{array}{l}\text { Sets columns in TotalResults.txt after evaluation; see } \\
\text { the text for more details. }\end{array}$ \\
\hline $\begin{array}{l}\text { BestPerAnalysisResult- } \\
\text { FileColumns: X }\end{array}$ & -bpaefc X & $\begin{array}{l}\text { Sets columns in BestPerAnalysis.txt after evaluation; } \\
\text { see the text for more details. }\end{array}$ \\
\hline OutputDirectory: $\mathrm{X}$ & -od $\mathrm{X}$ & Specifies directory in which the results should be stored. \\
\hline OutputExists: $\mathrm{X}$ & -oe X & $\begin{array}{l}\text { Specifies what to do if output directory already exists. } \\
\text { overwrite will delete existing output and overwrite it } \\
\text { with the new results. add will add the current results to } \\
\text { the old ones, see text. }\end{array}$ \\
\hline
\end{tabular}

Table 1: Summary of all parameters which can be set within CheckMATE, either via the parameter card introduced in Section 4 or as command line input./CheckMATE $-\mathrm{X}-\mathrm{Y}$... Occasional dash symbols $(-)$ in the first column only indicate that a command is split in two lines to reduce the column width and are not part of the keyword. ${ }^{*}$ The use of LHE events that have only been generated at the parton level or showered events that have been pre-clustered is not recommended and may lead to substantial efficiency and acceptance errors. 


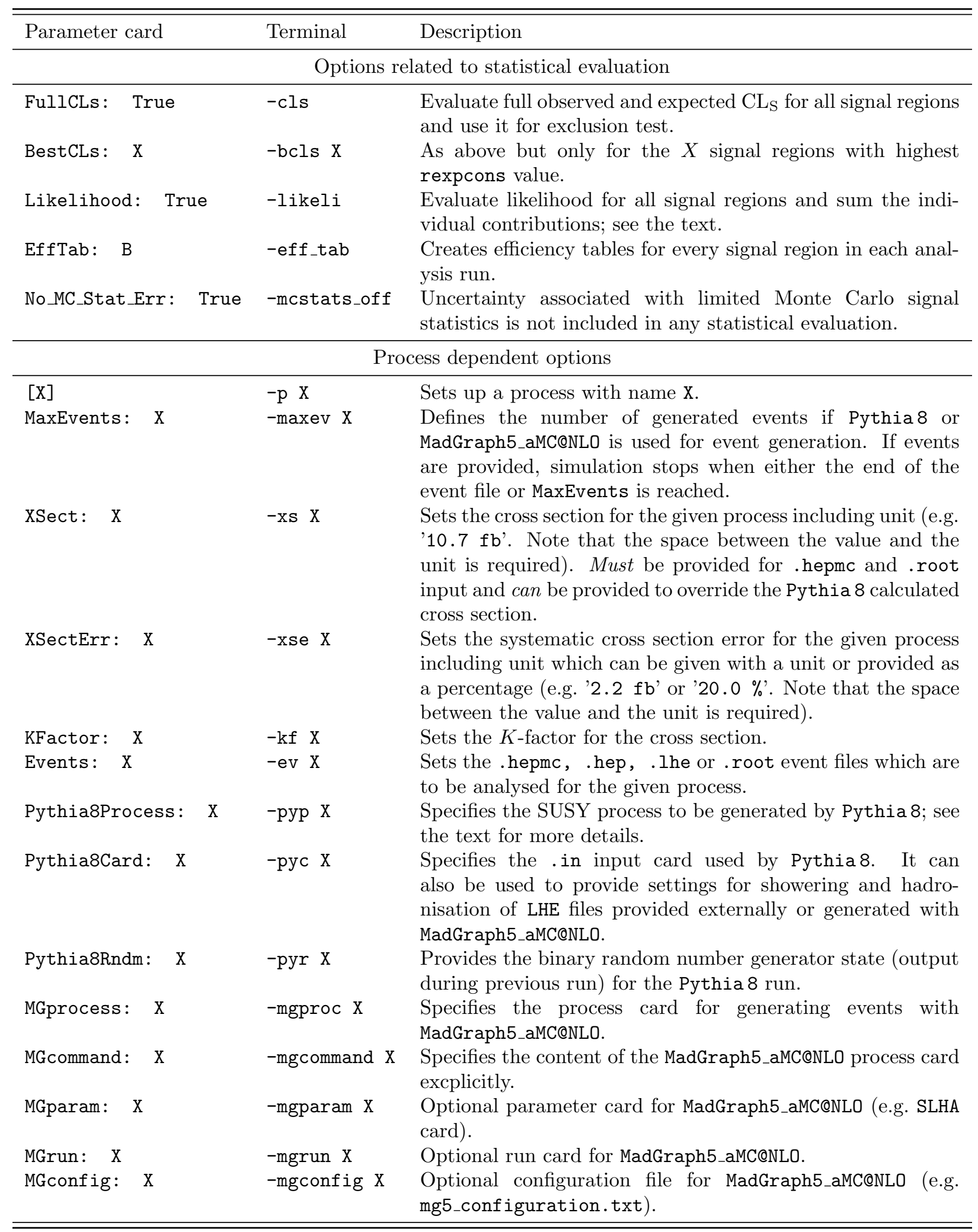

Table 2: Continuation of Table 1 Occasional dash symbols (-) in the first column only indicate that a command is split in two lines to reduce the column width and are not part of the keyword. 
which are mostly self-explanatory. The suffix - cons refers to the $r$ value in eq. (1) and columns without this suffix do not include the conservative subtraction term of $1.64 \cdot \Delta S$ in the numerator. Note that the output of $\mathrm{CL}_{\mathrm{S}}$ and likelihood related columns are set to -1 unless the calculation of the respective quantity is enabled via the corresponding flag; see Table 2.

add mode: After a CheckMATE run is completed, the user might realise that the events which were processed were insufficient. For example, the size of the tested Monte Carlo samples might be too small to find the number $S$ with desired statistical precision. It might also happen that a posteriori it becomes apparent that other processes need to be taken into account which were expected to be negligible before the first run. For those cases, CheckMATE allows for new results to be added to old ones. To do so, CheckMATE has to be set up with the same name and the same output directory as the original run. During the initialisation step the user is then explicitly asked if the new results are supposed to replace or to be added to the existing ones. If the second option is chosen, the original CheckMATE settings are restored from the earlier run, all events in the current setup file are processed and properly added to the ones of the first run. This procedure can be repeated as many times as the user wishes. This behaviour can be controlled by the OutputExists parameter or using -oe add from the command prompt.

Likelihood: Instead of exclusion tests, CheckMATE can also be used for model fits; see Ref. [59] for an example. With this option CheckMATE can calculate the likelihood ratios for all signal regions to test the compatibility of a given model with the experimental results. A formulae for a simplified LHC profile likelihood ratios without nuisance parameters is given by Eq. (2) in Appendix B.1. CheckMATE uses the full version including $\Delta B$ and $\Delta S$ as nuisance parameters given by Eq. (5) of Section B.1. In the final results, CheckMATE also returns the sum of the likelihood ratios over all signal regions but this value should be used with care since no checks for kinematically overlapping regions are considered. The user is advised to check the signal regions of interest and only sum those that are independent. Analysing the dependence of this quantity on model parameters allows one to find best fit points and the corresponding confidence intervals, see e.g. Ref. [58].

Pythia8Process: Due to the popularity of the MSSM, CheckMATE also allows one to easily set up generation of SUSY production processes using the Pythia8Process keyword. Possible values for this parameter are $\mathrm{p} \mathrm{p}>\mathrm{X}$, with $\mathrm{X}$ being any of the following:

go go: gluino pair production;

go sq: gluino-squark and gluino-antisquark associated production;

sq sq : squark-antisquark production;

t1 t1 : pair production of the lightest stop;

3gen: pair production of stops and sbottoms;

sq sq: squark pair production;

colsusy: all coloured SUSY pair-production;

ewsusy: pair production of neutralinos, charginos and neutralino-chargino production;

allsusy: all of the above.

Note that here "squark" always corresponds to the squarks of the first two generations. To simulate any other process or any combination of the above, an explicit Pythia 8 . in file has to be provided. For all processes the default parton distribution function [60] is used and the underlying event is switched off. 


\section{Example: Running CheckMATE and Understanding the Results}

To illustrate how the individual steps explained in Section 2 work in practice, we discuss an example CheckMATE run. It is designed in such a way that it covers the most common scenarios to provide input data within the current CheckMATE version. It also attempts to apply some optional settings to illustrate their meaning. After the example run is completed, we take a closer look at the auxiliary files which are created along the way and what additional information a user can find in these. A tarball containing all files which are used as input in this example run can be downloaded from http://www.hepforge.org/archive/ checkmate/ExampleFilesForCheckMATE2Manual .tar.gz

\subsection{Benchmark Model}

Within this section we test a simplified supersymmetric model where the only kinematically accessible particles are the gluino with mass $1.5 \mathrm{TeV}$, the eight mass-degenerate squarks of the first two generations with mass $1.5 \mathrm{TeV}$ and a $100 \mathrm{GeV}$ stable bino-like neutralino lightest supersymmetric particle (LSP). Here, the gluino is expected to always decay democratically ${ }^{13}$ into same-flavour quark-antiquark pairs of the first two fermion generations and the stable lightest neutralino. Squarks always decay into the associated Standard Model quark and the neutralino. The masses of squarks and gluino are chosen such that neither of the two can directly decay into the other. The important parts of the SLHA file for this model are as follows:

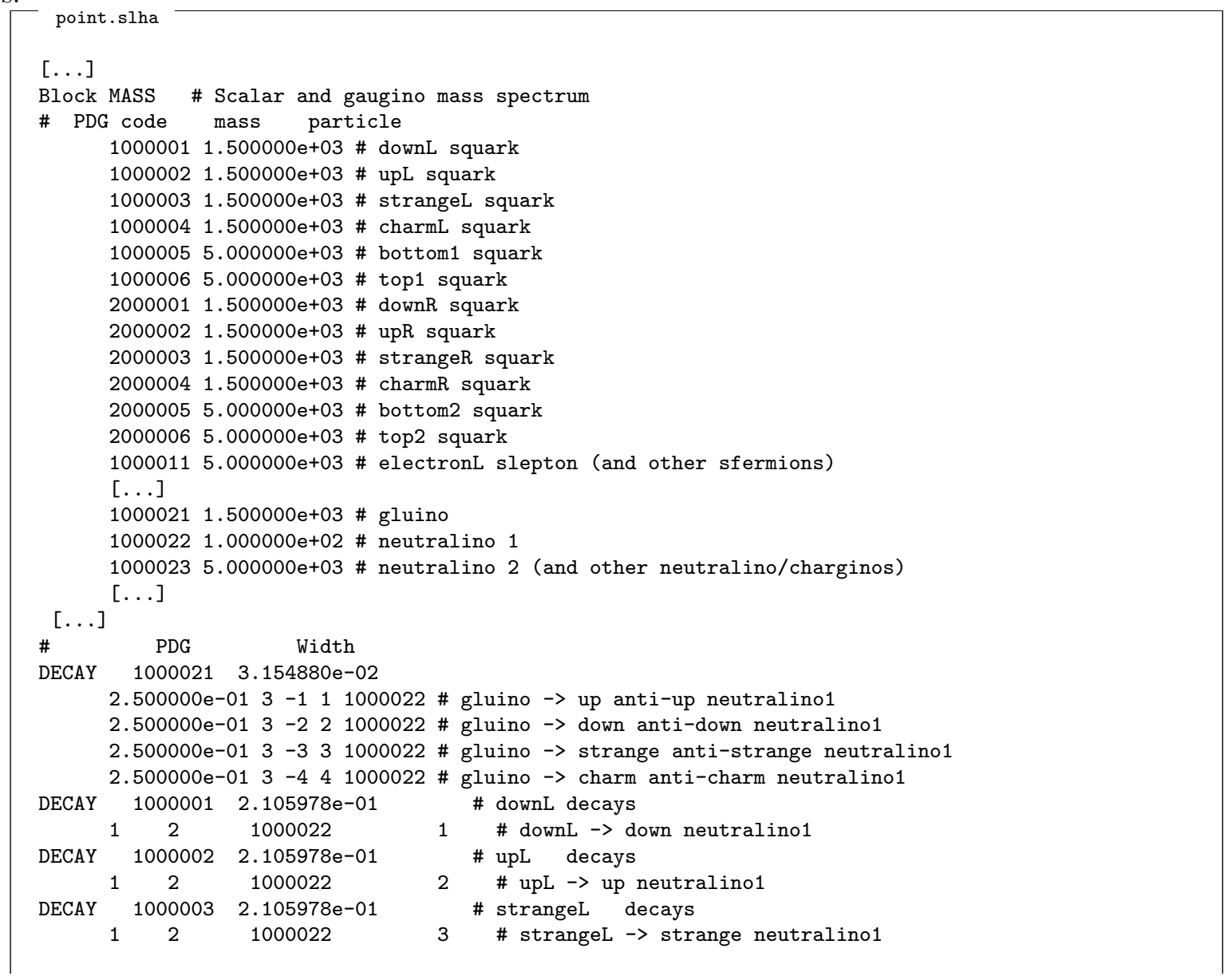

\footnotetext{
${ }^{13}$ For a bino-like LSP, a gluino would actually decay with different branching-ratios into up-like and down-like quarks due to their different quantum numbers. However, phenomenologically these quarks are almost indistinguishable at the LHC so we can safely set all branching ratios equal.
} 


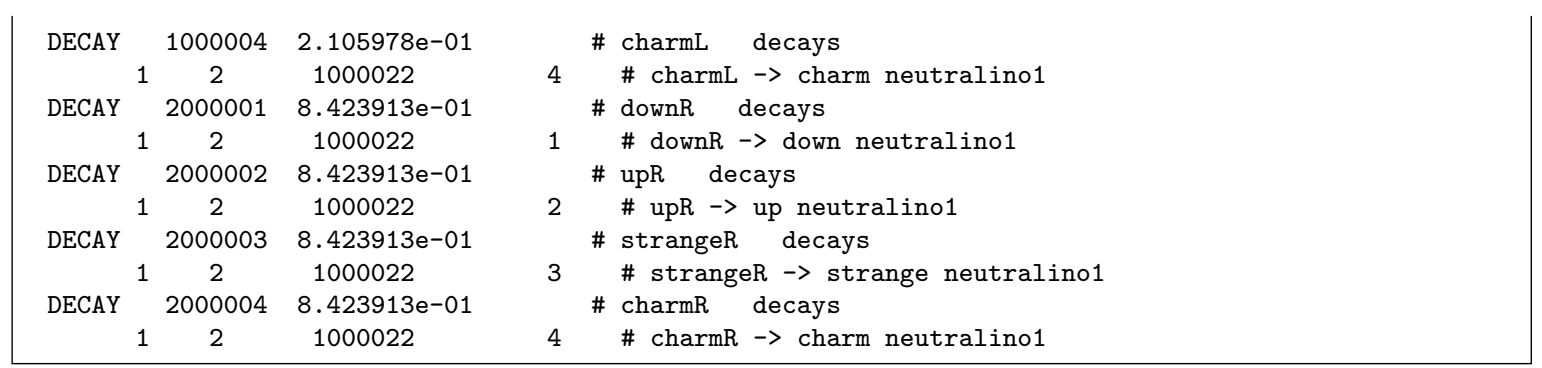

The most relevant production modes for such a model are the 2 -body final states $p p \rightarrow \tilde{g} \tilde{g}, \tilde{g} \tilde{q}, \tilde{q} \tilde{q}$ and $\tilde{q} \tilde{q}^{*}$. In our example run, we use different approaches ${ }^{14}$ to generate the events for these processes:

- For squark-antisquark production, $p p \rightarrow \tilde{q} \tilde{q}^{*}$, we call MadGraph5_aMC@NLO internally to do the parton level event generation and subsequently let Pythia 8 do the parton showering. This mode is one example of how to perform the event generation entirely on the fly. In our example we explicitly give the commands for MadGraph5_aMCONLO to simulate the correct final state.

- Similarly, for the final state $\tilde{q} \tilde{q}$ of squark pair production, we also generate events within CheckMATE, however this time we entirely rely on Pythia 8 to do both the partonic event generation and parton showering. Here, we setup Pythia 8 via its .in settings file.

- For events of type $\tilde{g} \tilde{g}$, we provide partonic . The files generated with MadGraph5_aMC@NLO beforehand and perform the parton showering and hadronisation with Pythia 8 directly within CheckMATE.

- Associated gluino-squark production has been performed completely externally and we provide two fully showered .hepmc files. The two files contain the same physics process generated with different random seeds such that they contain statistically independent samples. Such a setup with multiple files per process can for example be required when event generation was parallelised on a computing cluster.

\subsection{Initialising and Starting CheckMATE}

We assume that CheckMATE has already been properly installed in folder \$CMDIR including Pythia and MadGraph5_aMC@NLO functionalities; see also Appendix A. The pre-generated . Ihe file for the $\tilde{g} \tilde{g}$ and the .hepmc file for the $\tilde{g} \tilde{q}$ process are located in /scratch/files. To run CheckMATE, some mandatory information has to be provided, either via a command line input or via a text-based parameter card. As the former only works for runs with single process, we have to choose the second approach. A (not minimal) working example for the above setup reads as follows:

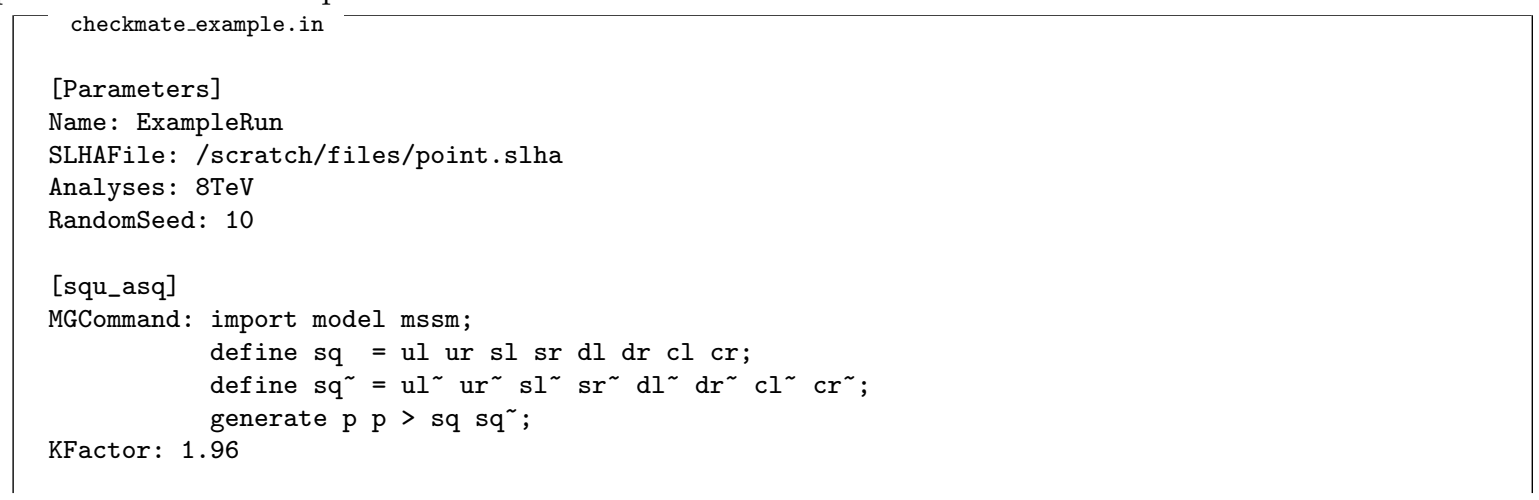

\footnotetext{
${ }^{14}$ The different input modes are only combined for illustrative purpose here. In practice, one would normally use the same tool setup for all the different hadronic SUSY final states.
} 
[squ_squ]

Pythia8Card: /scratch/files/pythiasqusqu.in

MaxEvents: 1000

[glu_glu]

Events: /scratch/events/glu_glu. The

XSectErr: $20 \%$

[glu_sq]

Events: /scratch/events/glu_squ_1.hepmc, /scratch/events/glu_squ_2.hepmc

XSect: $1.90 \mathrm{fb}$

The general structure of such a file consists of blocks separated by [] expressions which contain one or more Key: Value pairs.

The first such block, [Parameters], is a special block type which lists general settings for the CheckMATE run common to all processes. In our example, we first give our run a specific name ExampleRun which specifies the name of the output directory. With a nonzero RandomSeed we make the results deterministic as this parameter fixes the sequence of random numbers which is used to e.g. simulate kinematic configurations in the event generation and apply finite efficiencies on final state objects in the detector simulation phase.

We then provide the already explained .slha spectrum file which informs MadGraph5_aMC@NLO and Pythia 8 about the masses and decay tables of all SUSY particles. This file is common to all processes since obviously the same physics scenario should be considered within one CheckMATE run. In our case, the SLHAFile is a mandatory parameter as we ask CheckMATE to simulate the events internally and therefore not providing this parameter would result in an immediate abort ${ }^{15}$ Additional possible settings which can be changed via the [Parameters] block are summarised in Section 3.

Besides the special [Parameters] block, any other [X] block combines the information for a particular production process $\mathrm{X}$, where $\mathrm{X}$ is a freely chosen identifier. In our particular case, we need four such blocks for all the different production modes we wish to take into account. Within each such process block we have to provide the information that describes the form of the Monte Carlo events for the particular process given.

- We start with the block [squ_asq] responsible for $\tilde{q} \tilde{q}^{*}$ production. With the MGCommand keyword, we specify the set of commands to internally call MadGraph5_aMC@NLO. For our example, we load the Minimal Supersymmetric Standard Model via the import model mssm command, combine all squarks and all antisquarks into single identifiers sq, sq ${ }^{\sim}$, respectively and conveniently setup pair production of all squark-antisquark pairs via generate $\mathrm{p} \mathrm{p}>\mathrm{sq} \mathrm{sq}^{\sim 16}$ Since we do not specify otherwise, MadGraph5_aMC@NLO will be set up so that it simulates 5,000 partonic events for the given process. Because no explicit cross section is provided, CheckMATE uses the result from MadGraph5_aMC@NLO determined during the generation of the events. We use the optional KFactor parameter to specify a $K$-factor which we determined using NLLFast. It multiplies the MadGraph5_aMC@NLO leading order cross section with a fixed quantitiy to estimate the cross section at the next-to-leading order plus next-to-leading log accuracy in QCD.

- For the simulation of $\tilde{q} \tilde{q}$ pair production, we set up a second block called [squ_squ]. Here, we also want the event generation to be done entirely internally via Pythia 8, however this time we explicitly provide the .in setting file for Pythia.

\footnotetext{
${ }^{15}$ If we instead only provided showered .hepmc files, this parameter would not be mandatory as no model-dependent information would be required. Note that providing partonic . Ihe may require an SLHA file including the full decay table of all BSM particles, namely if the BSM final state particles are not yet fully decayed and if the decay table is not included in the header part of the . Ihe event file already.

${ }^{16}$ Note that this call will form all possible final state combinations of the product (ul $\mathrm{ur}_{\mathrm{dl}} \ldots$ ) $\times(\mathrm{ul} \sim \mathrm{ur} \sim \mathrm{dl} \sim \ldots$ ), including flavour-nondiagonal pairs, because of different combinations of initial state quarks in protons.
} 


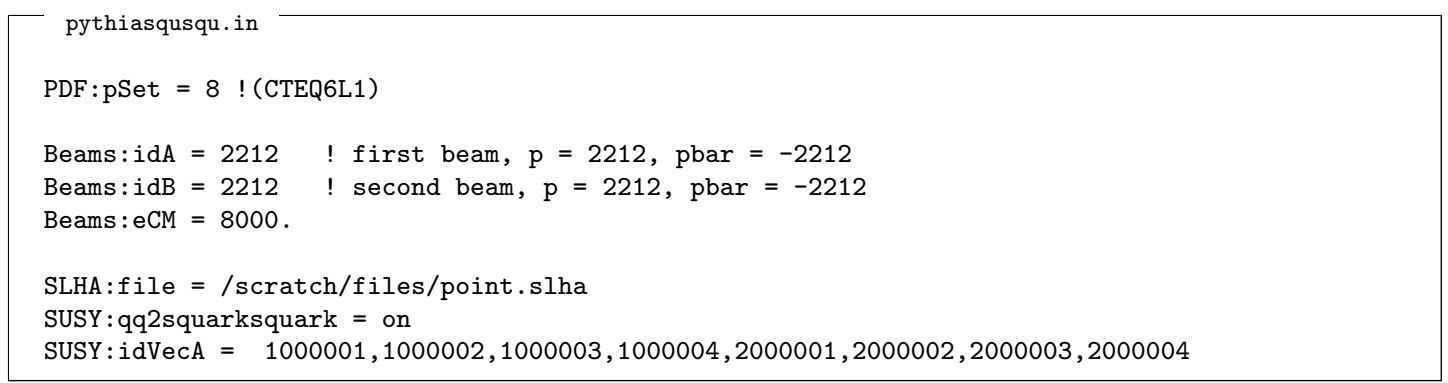

The meaning of the individual lines should be self explanatory. The last row specifies the set of squarks which should be taken into account and which we set to all left- and right-chiral squarks of the first two generations. In principle, any of Pythia 8's parameters listed in Ref. [53] can be changed via this file. Note that for this block, we explicitly specify the number of generated events to be 1,000 via the optional parameter MaxEvents.

- The third process block [glu_glu] sets up the gluino pair production process where we have already generated 1,000 events using MadGraph5_aMC@NLO. Here, we simply have to provide a reference to this file via the Events keyword. There is no other mandatory parameter in this case. Most importantly, CheckMATE uses standard Pythia settings for showering and hadronisation, see below, and takes the cross section from the . Ihe file itself. In this example, we provide the optional parameter XSectErr to inform CheckMATE about the systematic error it should consider for this process, which we assume to be $20 \%$ of the signal cross section. If no such parameter is provided, as we do for the other three processes, the systematic error associated with the signal is set to zero.

- Finally, we provide two fully hadronic .hepmc files for associated gluino squark production in the [glu_squ] block. We can simply list all available files for a given process in one Events command and in the end the results of all files are properly averaged as explained below. If CheckMATE is run with showered .hep or .hepmc files the cross-section must be provided explicitly by the user as contrarily to the . Ihe format, these event formats do not store this information. In our case, the provided cross section is taken from Pythia 8 which we used to simulate the events but we could have instead used NLLFast or Prospino.

With the above files ready, we can start CheckMATE with the following command 17

\$CMDIR/bin: ./CheckMATE checkmate_example.in

CheckMATE then responds with a summary of the used settings for the given run and asks a user for confirmation.

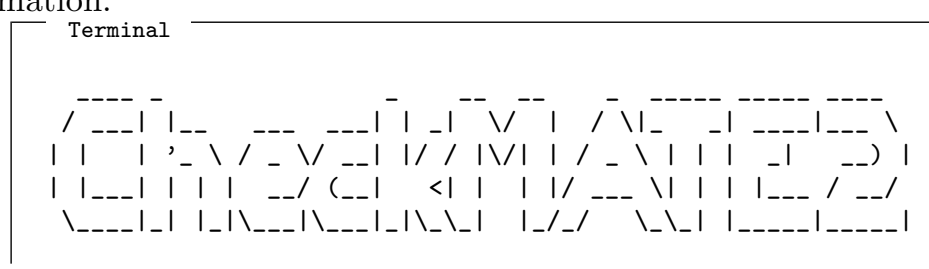

\footnotetext{
${ }^{17}$ Within this chapter, gray text denotes input to be entered by a user.
} 
The following settings are used:

Analyses:

cms_1301_4698_WW (WW production only, $8 \mathrm{TeV}, 3.5 \mathrm{fb}-1$ )

cms_1303_2985 (CMS, alpha_T + b-jets)

$[\ldots]$

atlas_conf_2014_056 (Constraint on stop production from ttbar spin correlations)

atlas_conf_2015_004 (Search for an invisibly decaying Higgs boson produced via vector ...

E_CM: 8.0

Processes:

Process Name: squ_asq

Input KFactor: 1.96

Associated event files and/or Monte-Carlo generation runs: MG5_aMCNLO Events

- internal identifier: 'squ_asq'

- command: import model mssm;

define $\mathrm{sq}=\mathrm{ul}$ ur $\mathrm{sl} \mathrm{sr} d \mathrm{dl} \mathrm{dr} \mathrm{cl} \mathrm{cr}$;

define $\mathrm{sq}^{\sim}=\mathrm{ul}^{\sim} \mathrm{ur}^{\sim} \mathrm{sl}^{\sim} \mathrm{sr}^{\sim} \mathrm{dl}^{\sim} \mathrm{dr}^{\sim} \mathrm{cl}^{\sim} \mathrm{cr}^{\sim}$;

generate $\mathrm{p} \mathrm{p}>\mathrm{sq} \mathrm{sq}^{\sim}$

Process Name: squ_squ

Associated event files and/or Monte-Carlo generation runs:

Pythia8 Events

- internal identifier: 'squ_squ'

- .in settings file: /scratch/files/pythiasqusqu.in

- at most 1000 events are generated and analysed

Process Name: glu_glu

Input cross section error: $20.0 \%$

Associated event files and/or Monte-Carlo generation runs:

LHE Events

- internal identifier: 'glu_glu'

- path to . Ihe file: /scratch/events/glu_glu.lhe

Process Name: glu_sq

Input Cross section: $1.9 \mathrm{fb}$

Associated event files and/or Monte-Carlo generation runs:

HepMC events

- internal identifier: 'glu_sq_event1'

- path to eventfile:/scratch/events/glu_squ_1.hepmc

HepMC events

- internal identifier: 'glu_sq_event2'

- path to eventfile:/scratch/events/glu_squ_2.hepmc

Output Directory:

\$CMDIR/results/ExampleRun

Additional Settings:

- SLHA file/scratch/files/point.slha will be used for event generation

- Fixed random seed of 10

Is this correct? $(\mathrm{y} / \mathrm{n})$

Here we chose that CheckMATE generates events at $\sqrt{s}=8 \mathrm{TeV}$ centre-of-mass energy and tests against all implemented ATLAS and CMS analyses for that particular energy. Note how a unique internal identifier is given to each process which will help us to associate output files to the corresponding input events later. In the following, when we use an expression event, it is to be understood as a placeholder for one such internal identifier.

As soon as we start CheckMATE by answering y, it informs us that - since we did not specify otherwise 
- MadGraph5_aMC@NLO is set up to generate 5,000 events for the squ_asq process.

Terminal

squ_asq:prepare(): Setting number of to-be-generated MC events to 5000 .

Use the 'maxEvents' Parameter to change this default behaviour.

After about five to ten minutes, depending on user's CPU, during which CheckMATE continuously updates us with the current status of the analysis chain, it returns the following result:

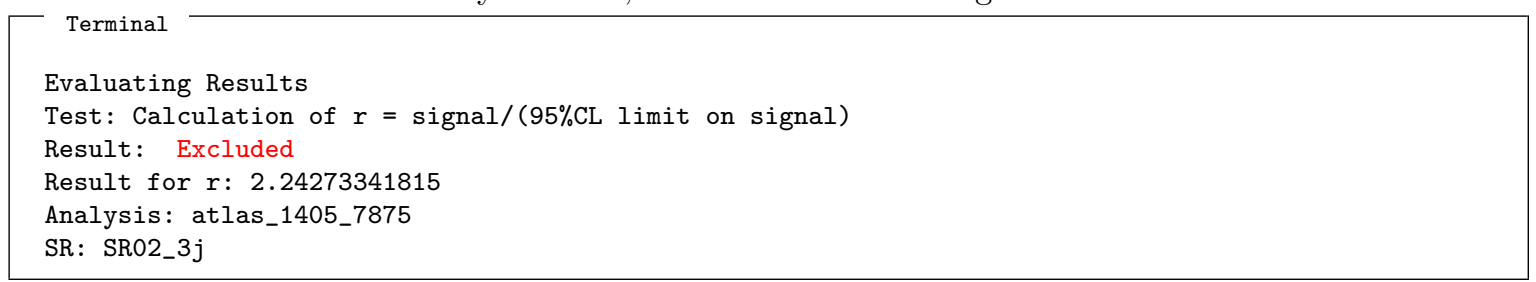

We find that after simulating all events, passing them through a detector simulation and performing 40 different analyses, CheckMATE concludes that the input parameter point is excluded because in signal region SR02_3j of analysis atlas_1405_7875, see Ref. 61], the number of predicted signal events $S$ exceeds the $95 \%$ upper limit $\mathrm{S} 95$ when testing the conservative value,

$$
r=\frac{(S-1.64 \cdot \Delta S)}{S 95}
$$

This agrees with the result of the experimental collaboration, see Figure 9 of Ref. 61.

For most users, this information would be sufficient for checking exclusion status of a given model. Simply by changing and testing different values of $m_{\tilde{q}}$ and $m_{\tilde{g}}$ given in the SLHA file, one easily finds the allowed and excluded regions in parameter space.

\subsection{Structure of the Results Folder}

We now take a closer look at the additional information that is stored in many files in the results folder. These files may be ignored by a user who simply performs a test for a given parameter point. However, knowing which information can be found in these files can be very helpful if for instance a more detailed breakdown of intermediate results is required or if CheckMATE behaves in an unexpected way. Furthermore, analysing these files aids us in understanding how CheckMATE internally works.

For our example case, the results folder would be located under $\$ C M D I R /$ results/ExampleRun. It contains the following files and directories:

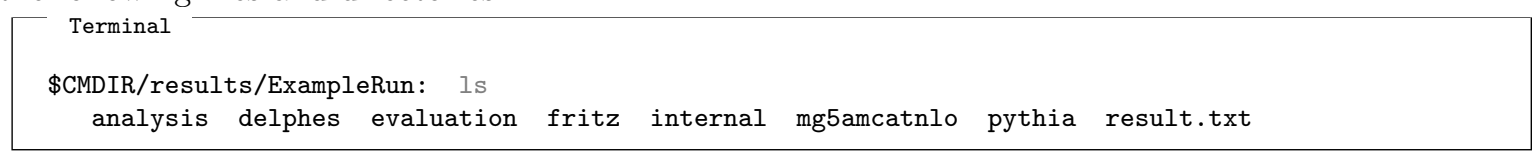

The file result.txt stores exactly the same information as printed on screen at the end of the CheckMATE run. The other folders store the respective individual information of the different modules as explained in the introductory section and we discuss them in the same order.

\subsubsection{Folder internal/}

The internal folder stores all internally set CheckMATE parameters in a Python readable format such that a posteriori one is capable of reproducing the exact Python instance of CheckMATE and is required if CheckMATE is run in the add mode explained in Section 3. In normal use they are of no relevance to a user and therefore we do not further discuss them here. Note that files in this folder are only created if CheckMATE finished successfully and did not abort due to an internal error. 


\subsubsection{Folder fritz/}

As FRITZ is the steering code which runs and calls the respective submodules, we continue our discussion with a content of the folder fritz. After running our example it should contain the following files:

Terminal

\$CMDIR/results/ExampleRun/fritz: Is

fritz_error.log fritz_glu_sq_event2.log glu_glu.ini squ_asq.ini

fritz_glu_glu.log fritz_squ_asq.log glu_sq_event1.ini squ_squ.ini

fritz_glu_sq_event1.log fritz_squ_squ.log glu_sq_event2.ini

Here, the fritz_event.log files contain the runtime output of FRITZ which was also printed on-screen while CheckMATE was analysing event. fritz_error.log combines the standard error output of all runs and should hopefully be empty at all times. Note that - besides the fritz_error.log file - there exists one . log file for each of the individually tested event files. Therefore, in our case, we have five files as we have four processes including one which uses two separate .hepmc event files. The respective .log files informs a user about the order in which individual modules were initialised, combined and finalised in the end, for example:

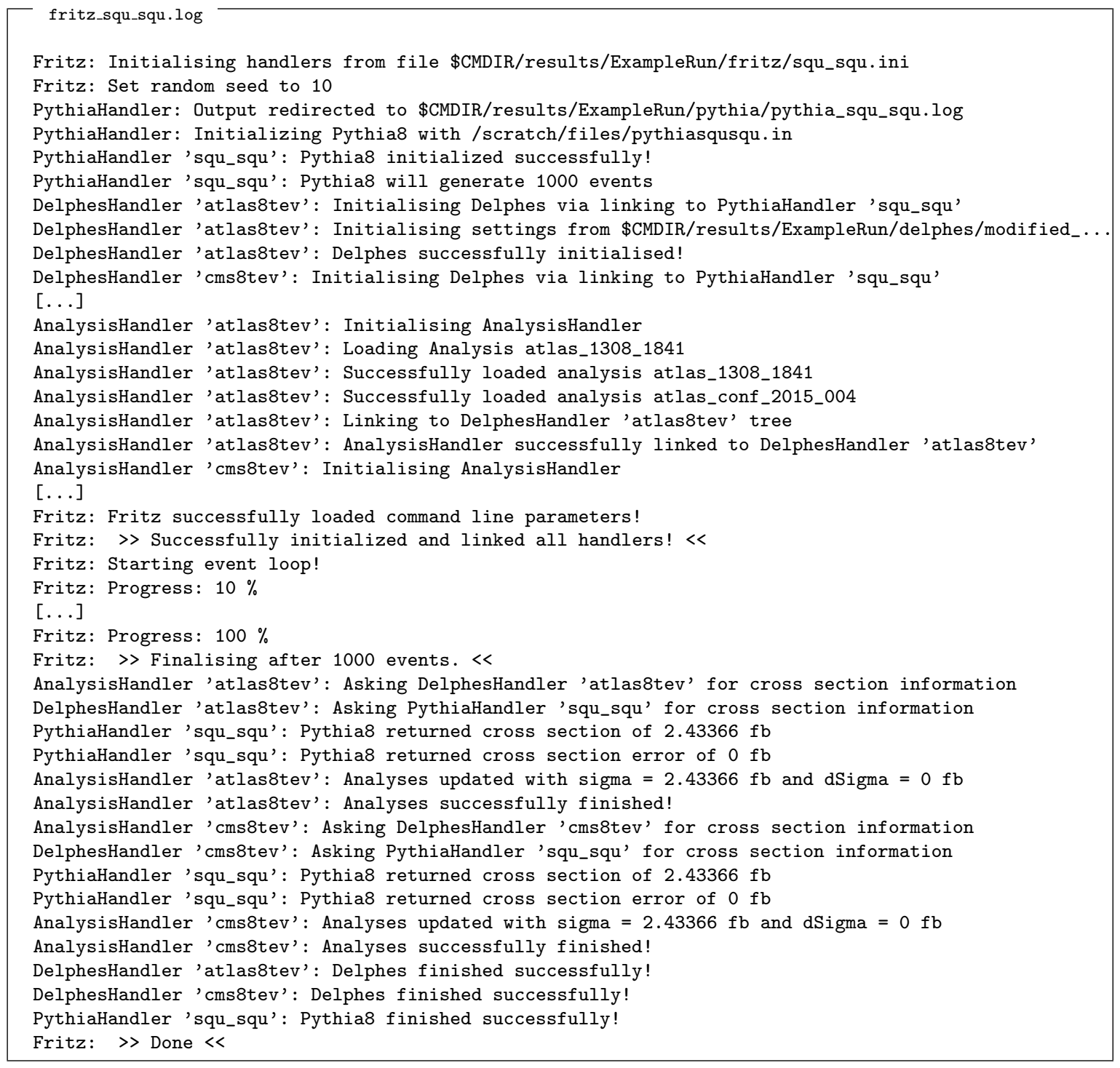


This file enables us to trace exactly which modules have been loaded using which settings and how they were respectively linked. In our particular example, for the squark pair production process we need a PythiaHandler which takes care of the event generation within Pythia 8 ${ }^{18}$ Then, since we test against all $8 \mathrm{TeV}$ analyses, we require two separate Delphes instances, one for the ATLAS and another one for the CMS detector description. Each of these DelphesHandlers takes its event information from the same single PythiaHandler, i.e. the same generated events are used for both ATLAS and CMS. The detector level objects are then respectively passed to two individual AnalysisHandlers which perform flavour tagging and final state isolation checks according to the requirements of the respective analyses. These then pass the information to all the loaded analyses which independently perform the signal region categorisation. In the end, the cross section information - which is needed by the individual analyses to properly normalise their final results - in this particular case is taken from Pythia 8 itself. Note that the cross section error from the event generator is set to zero, even though this value - as can be seen in the respective output files showed below - has a numerical uncertainty. This statistical uncertainty is however already accounted for by CheckMATE internally. Any additional systematic uncertainty has to be provided by the user via the XSectErr keyword.

The required set of handlers, their properties and how they are linked is determined by the Python part of CheckMATE and for each event is passed via an event.ini file, for example:

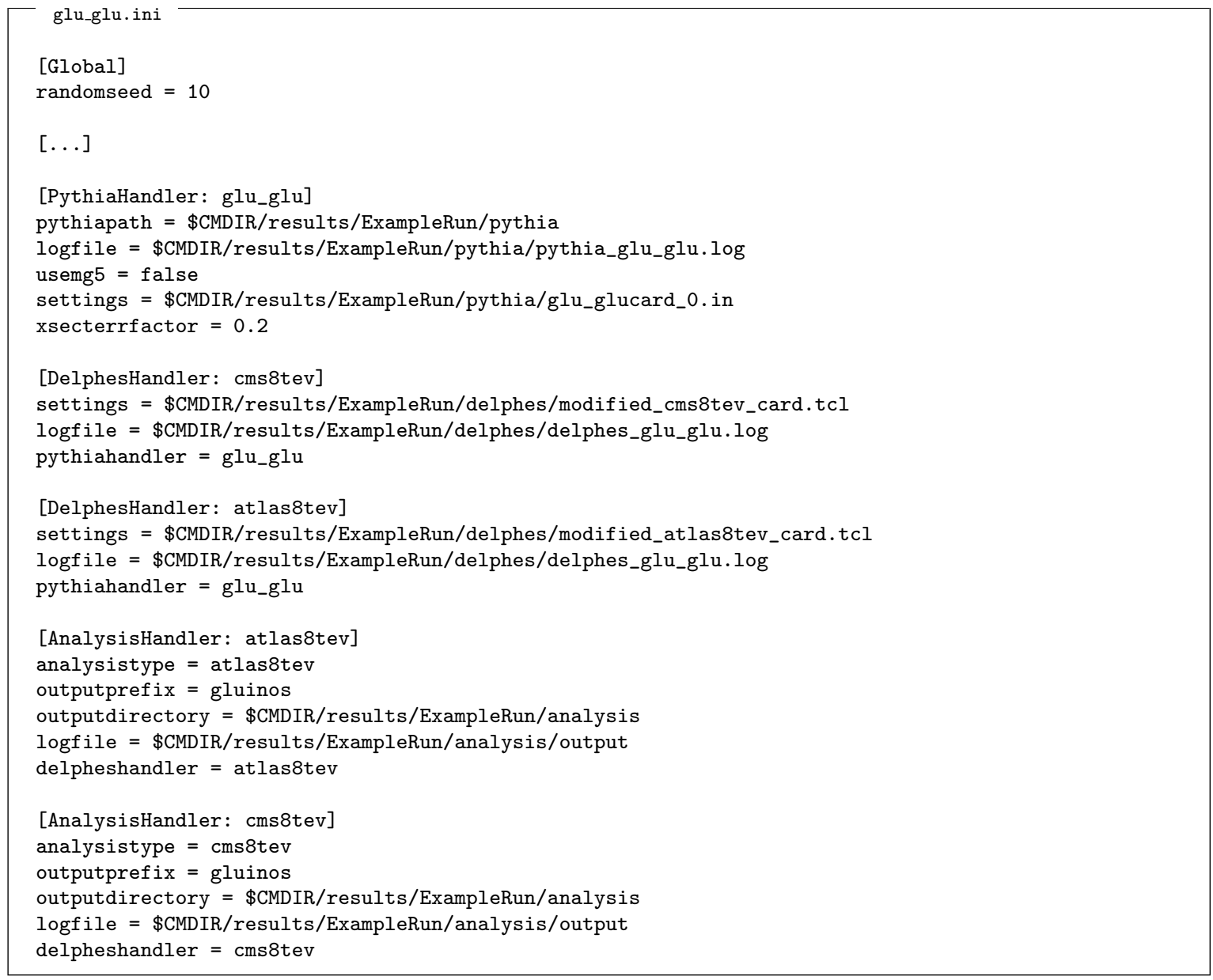

The two AnalysisHandlers responsible for ATLAS and CMS analyses need to apply flavour tagging and isolation conditions after the detector simulation step. As explained before, CheckMATE first analyses the

\footnotetext{
${ }^{18}$ Note that for practical purposes, each process starts a separate FRITZ run. This is why only one Pythia 8 instance appears in the above example logfile for the gluino run.
} 
respective analysis implementation in order to create a list of all required settings for each analysis. All this is stored in the same .ini file:

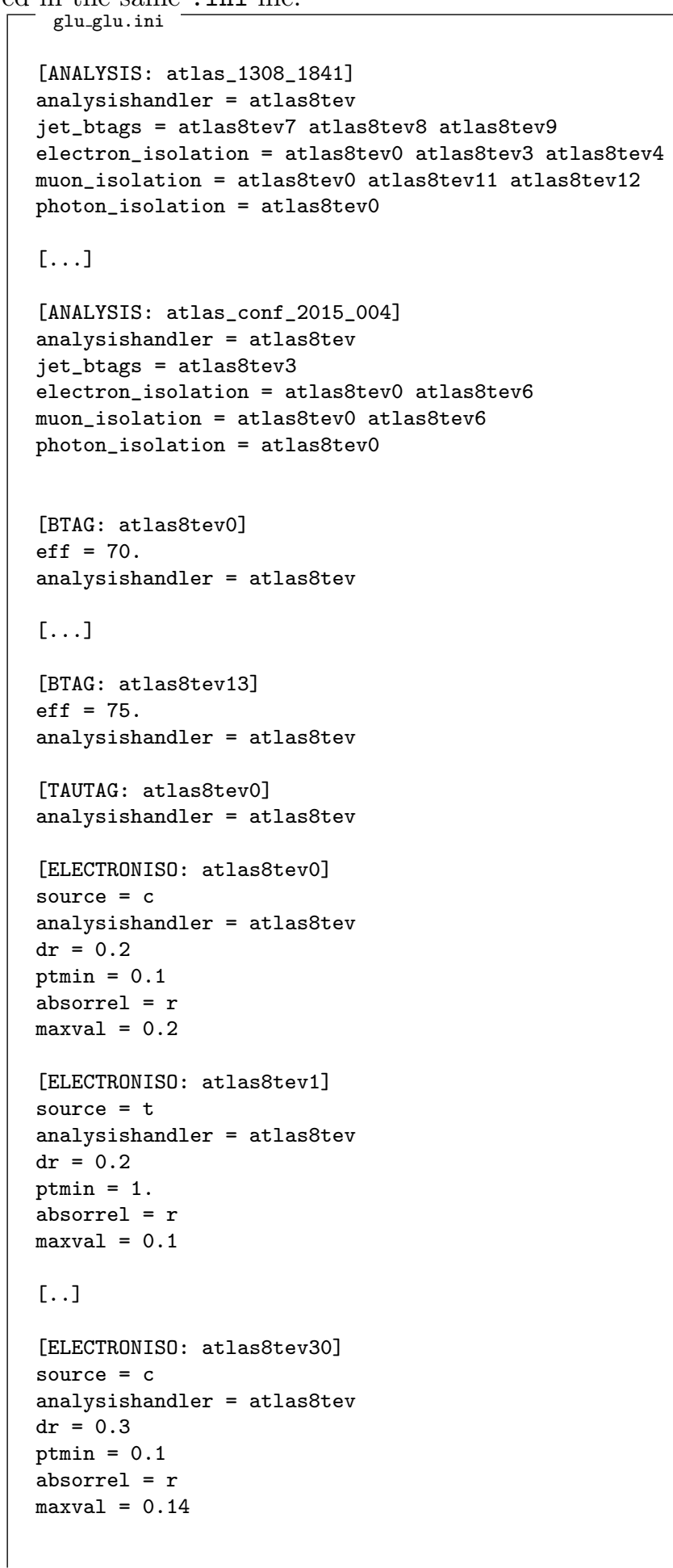




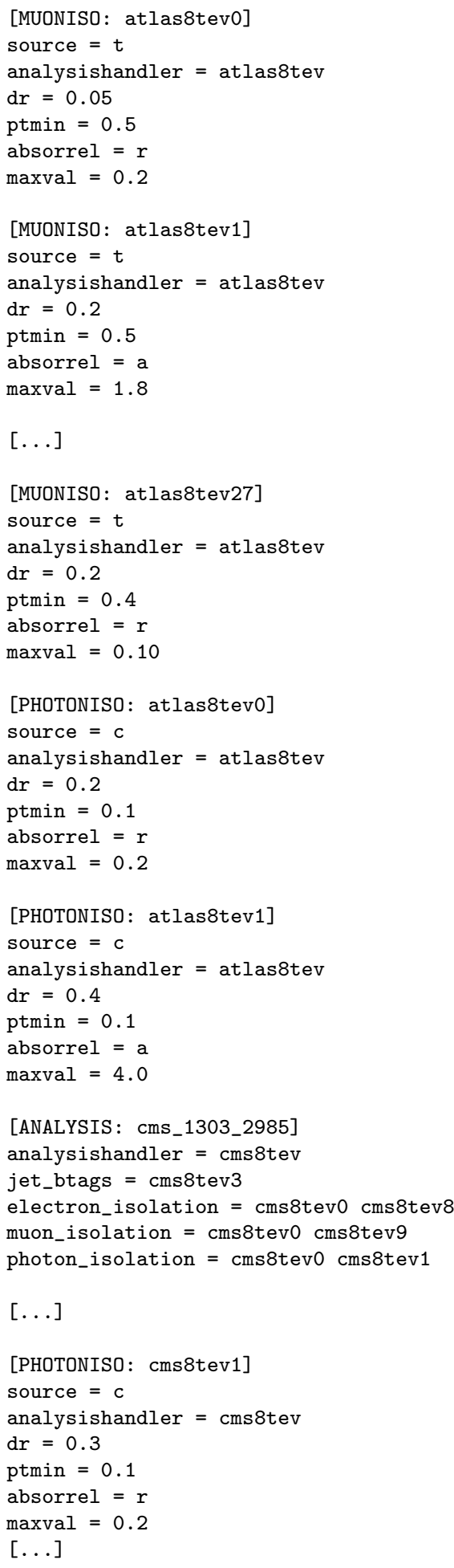

We find, for example, that the 31 ATLAS analyses (including those that are only partially validated, cf. Section 5 of our general $8 \mathrm{TeV}$ run require in total 30 distinct isolation tests for electrons, 27 different muon isolation tests and 13 different $b$-tagging working points. 
The flexibility of FRITZ becomes apparent when inspecting the corresponding .log file for e.g. the $\tilde{g} \tilde{q}$ process for which we provided the fully hadronised .hepmc files:

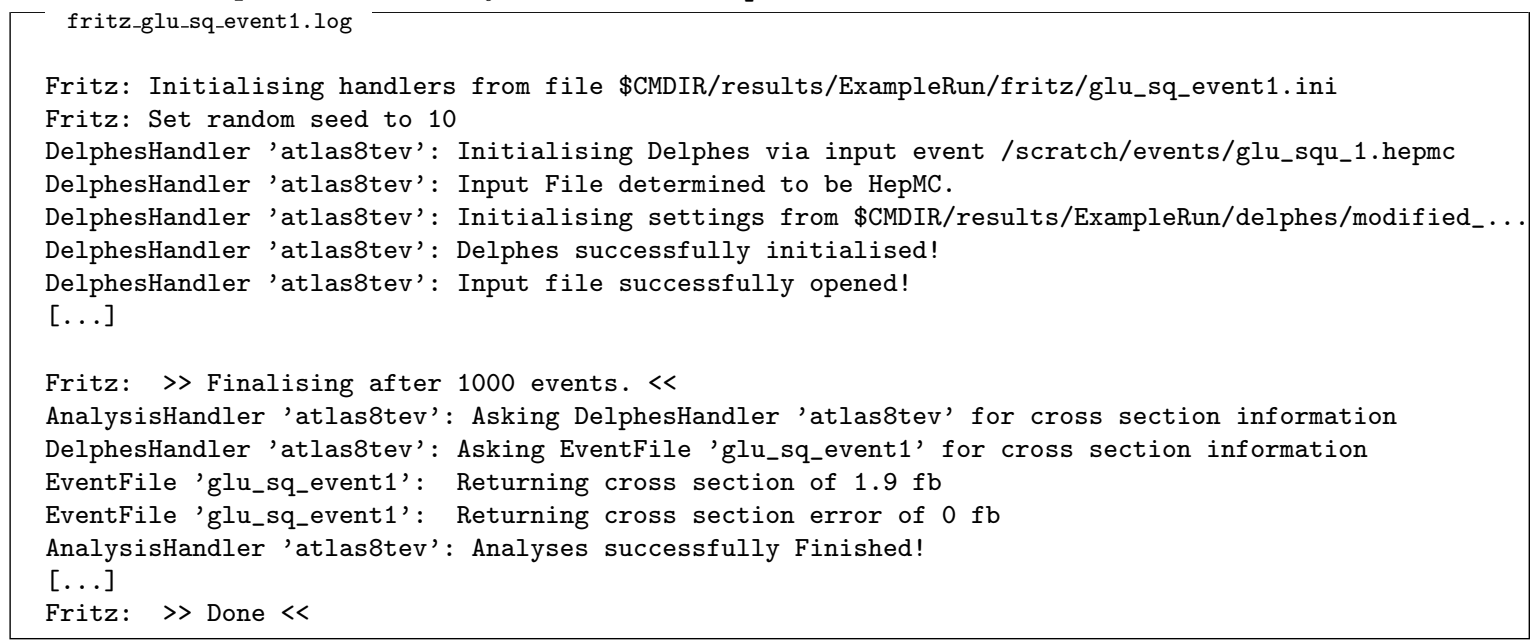

One finds that no PythiaHandler is loaded in this case. The .hepmc files are directly loaded into Delphes and the cross section is taken from a user which is passed to FRITZ and then to the analyses via the corresponding . ini file:

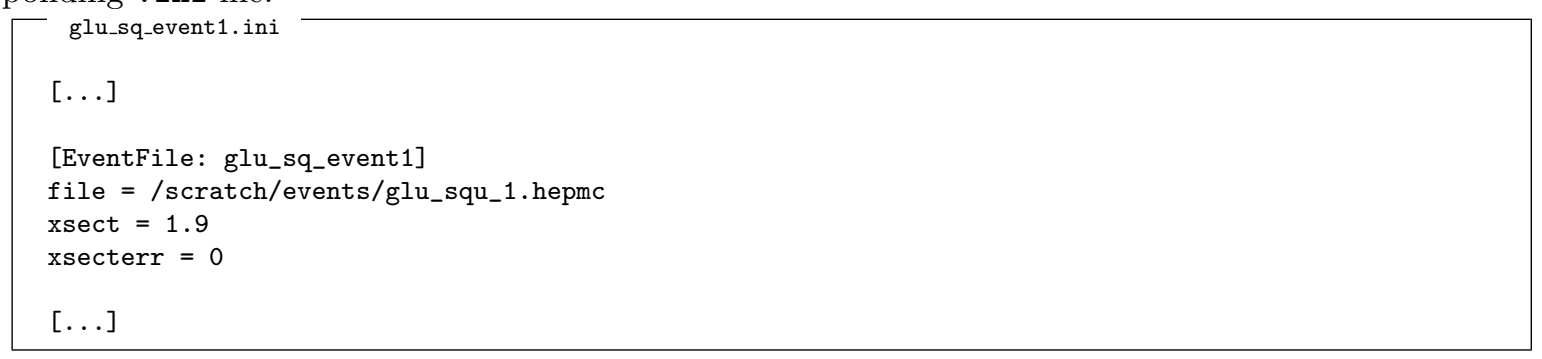

The analysis part, however, is the same for all processes.

\subsubsection{Folder mg5amcatnlo/}

Results of the event generation by MadGraph5_aMC@NLO are stored in this folder. We get the following files and directories:

\$CMDIR/results/ExampleRun/mg5amcatnlo/: ls

mg5amcatnlo_squ_asq.log squ_asq squ_asq_proc_card.dat squ_asq_run_card.dat

The names of all files and directories can be associated to the process that was generated via the respective unique identifier. In our case, we only encounter the identifier squ_asq as we enabled interal event generation with MadGraph5_aMC@NLO only for this process.

A typical MadGraph5_aMC@NLO run requires three files:

- A proc card lists the commands which should be given to MadGraph5_aMC@NLO to simulate the correct events. In our case, the file squ_asq_proc_card.dat contains the exact command we provided via the MGCommand parameter in our initial CheckMATE input file.

- A param card typically defines the parameters of the to-be-analysed BSM model in the form of an SLHA file. In our case, this file is already existing and was defined by the SLHA command. It, therefore, does not appear as a separate file in this folder.

- A run card specifies the details of the partonic Monte Carlo simulation. This file is created by CheckMATE, taking a standardised run card and filling it with information given by a user, e.g. the number of requested events and the centre-of-mass energy. 


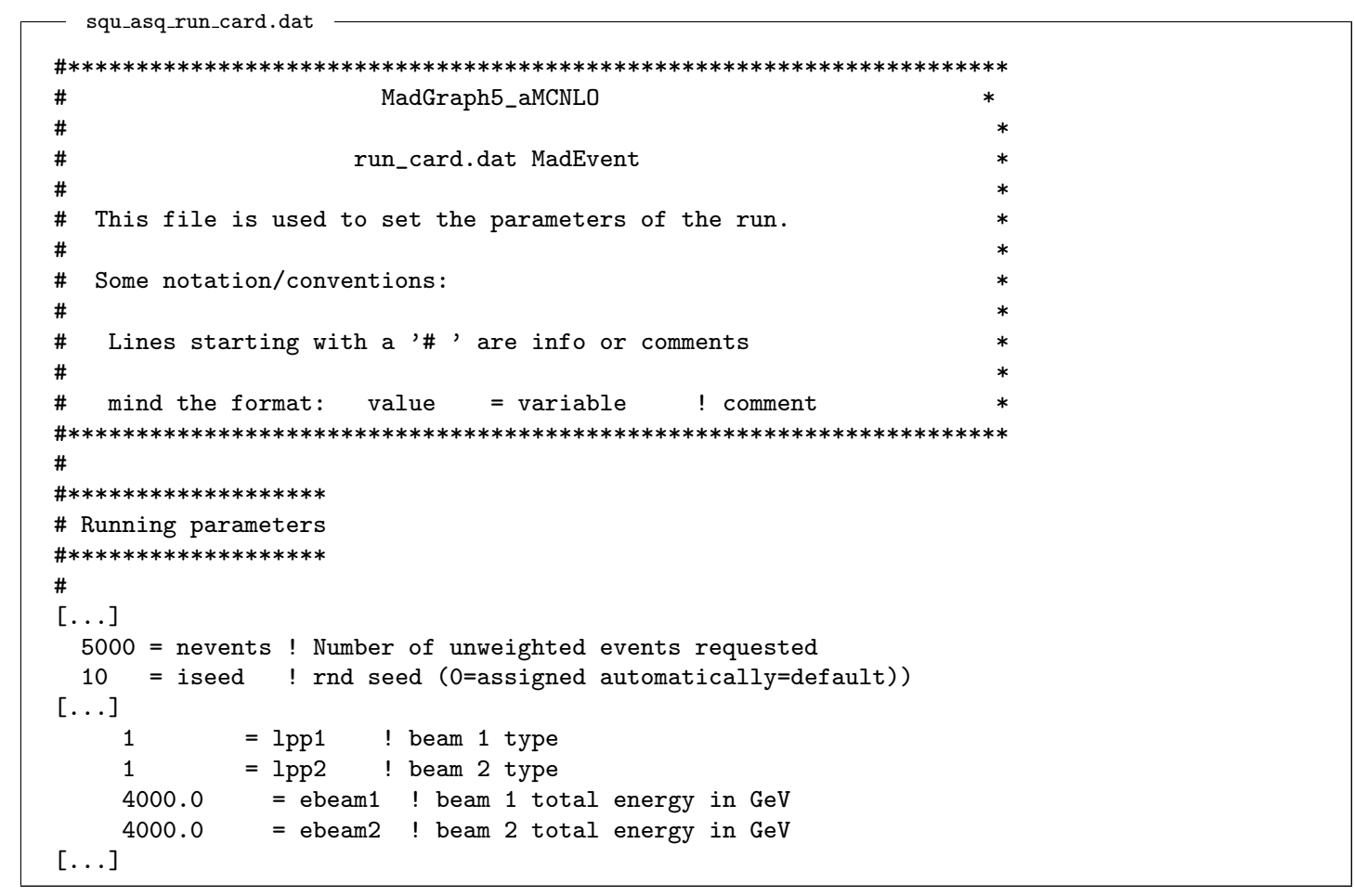

The squ_asq folder is the working directory of MadGraph5_aMC@NLO which contains all input files, executables and output files. For more information regarding the meaning of these we refer to the original publication, Ref. [6].

\subsubsection{Folder pythia/}

The pythia folder contains all files that have been used to simulate the events with Pythia 8. In our particular case, these are

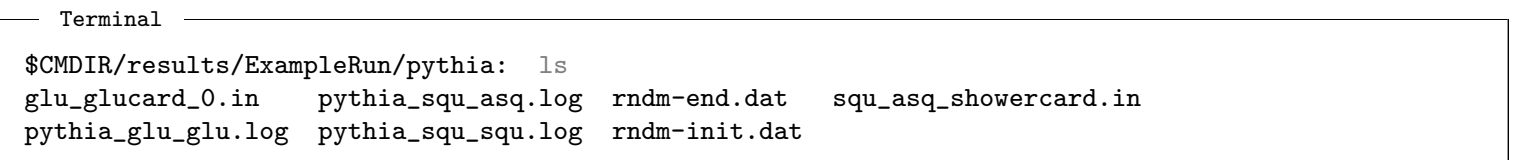

The . in files correspond to setup files for Pythia 8 which have been created by CheckMATE and which contain the commands to simulate the respective final state with the correct centre-of-mass energy using the SUSY parameter point from the .slha file. We have such a card for the [squ_asq] process, for which we used the MGCommand parameter. Here, CheckMATE generates the .in file used for showering the parton events from MadGraph5_aMC@NLO automatically.

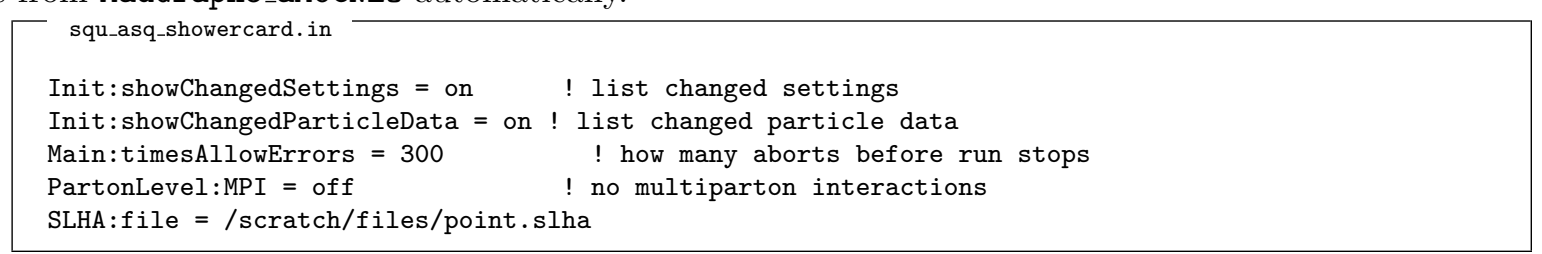

For the $\tilde{g} \tilde{g}$ production, we also have a card which sets up Pythia 8 for hadronisation of . Ihe events: 


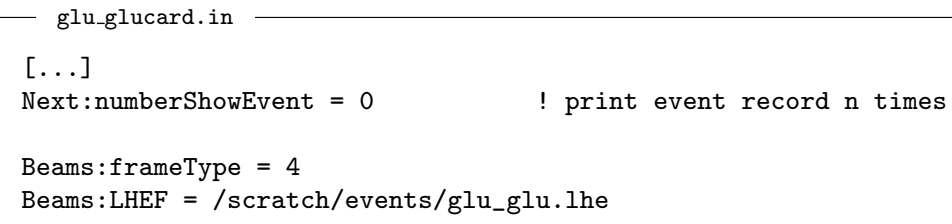

The .log files contain the verbatim output produced by Pythia 8 before, during and after the event generation. If the simulation finished successfully, this file concludes with a summary of the numerically evaluated cross sections, for example:

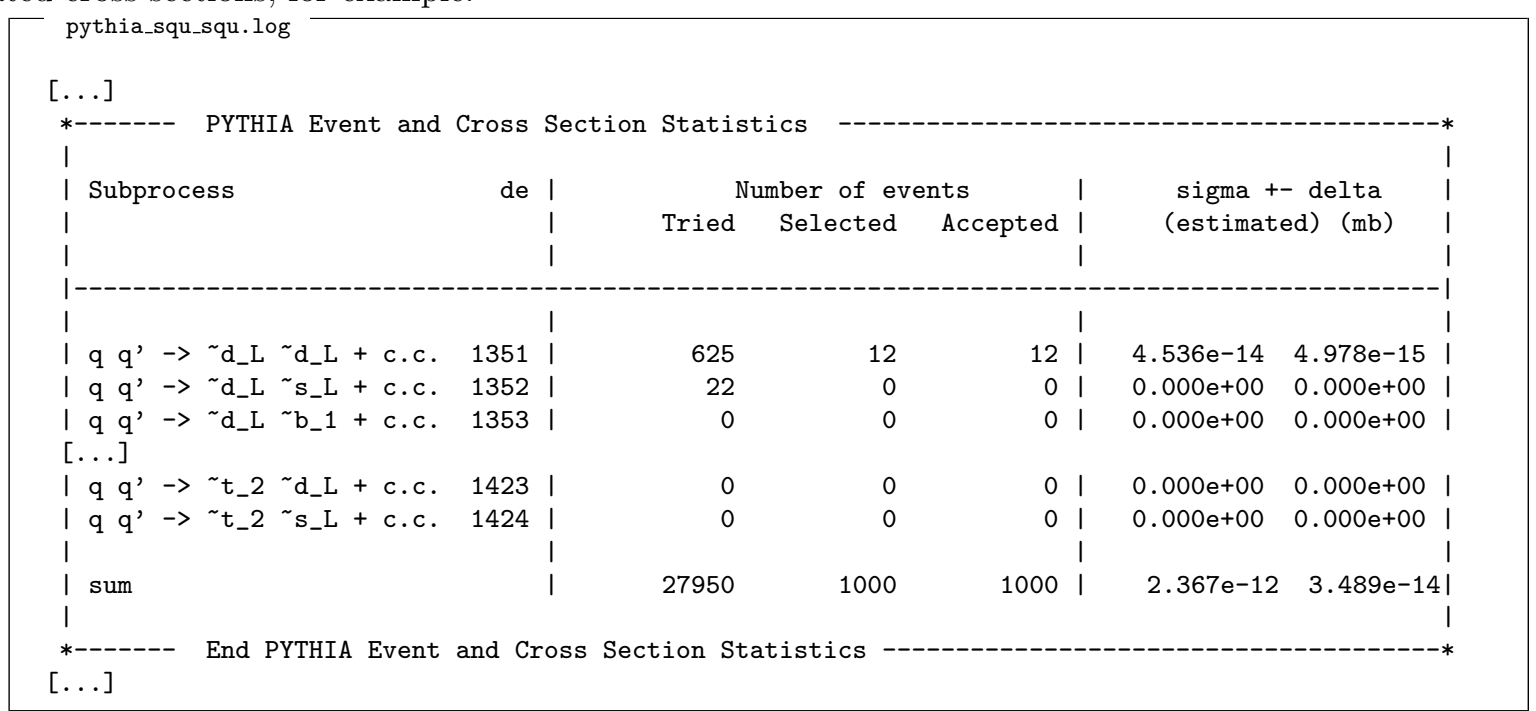

Note that, as expected, the final numbers coincide with the cross section values quoted in the fritz_squ_squ. log file that have been used for the analysis normalisation. If, for some reason, the event generation has to be aborted, this file can contain more information about the cause.

The run also produces two binary files rndm-init.dat and rndm-end. dat which contain the state of the random number generator. The random number sequence can be reproduced by providing Pythia8Rndm: $<$ path $>$ /rndm-init.dat in the parameter file. Alternatively, providing rndm-end. dat ensures that the new random number sequence is independent. If a user requires the directory to be overwritten, these files should be first copied to another location.

\subsubsection{Folder delphes/}

Analogously to the pythia folder, intermediate results of the detector simulation step are stored in the delphes folder.

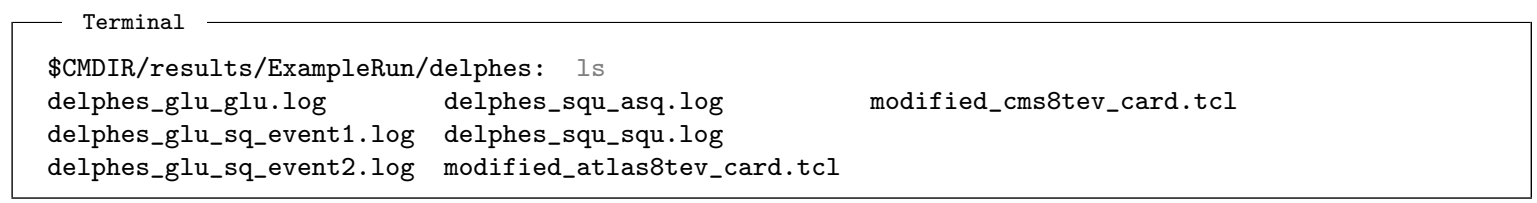

For our example, this folder simply contains the log files produced by Delphes for each of the five independent event files. These do not contain any interesting information if a run succeeded but can be of assistance if the detector simulation encountered an unexpected problem. Typically, CheckMATE uses standardised .tcl detector cards which can be found in data/cards within the CheckMATE installation directory. Only in our specific case, since we fix the random seed which can only be done in Delphes in the .tcl files, CheckMATE produces modified_*.tcl cards. The only difference to the standard cards is the appearance of the line set RandomSeed 10 at the very end. For more information about the fast detector simulation Delphes, we refer to Ref. [1]. 


\subsubsection{Folder analysis/}

A closer look into the analysis folder reveals a plethora of files.

terminal

\$CMDIR/results/ExampleRun/analysis: 1

glu_glu_atlas_1308_1841_cutflow.dat glu_sq_event2_atlas_conf_2013_024_signal.dat

glu_glu_atlas_1308_1841_signal.dat

$[\ldots]$

glu_sq_event2_atlas_conf_2012_147_signal.dat

glu_sq_event2_atlas_conf_2013_021_signal.dat

glu_sq_event2_atlas_conf_2013_024_cutflow.dat

squ_squ_cms_sus_13_016_cutflow.dat

squ_squ_cms_sus_13_016_signal.dat

To be precise, there are two files for each of the analysis for each process event file which in our case sums up to almost 400 different files. The content of these files has not changed since the original CheckMATE publication in Ref. [26]. For completeness, we discuss the logic and content of these files here.

Each analysis in CheckMATE produces two types of output: cutflow-files show the absolute and relative numbers of events that pass the individual selection cuts of the corresponding analysis step-by-step, whereas signal-files give the final number of events that pass all signal region cuts defined within the analysis. As shown below, both files have a common structure. For a detailed discussion we choose the analysis atlas_1405_7875 which CheckMATE determined to be responsible for the signal exclusion:
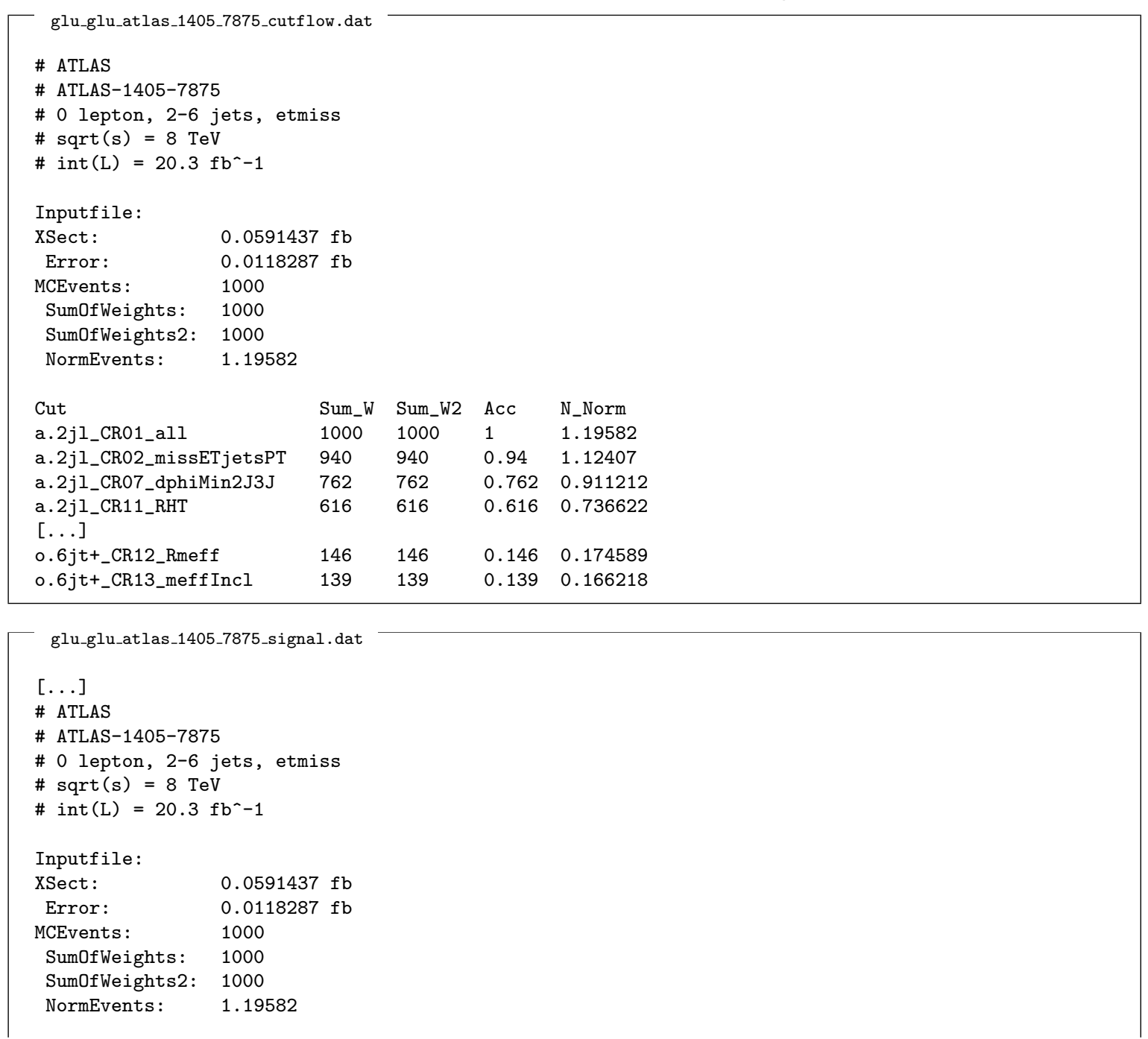


\begin{tabular}{|lllll|} 
SR & Sum_W & Sum_W2 & Acc & N_Norm \\
SR01_a.2j1 & 616 & 616 & 0.616 & 0.736622 \\
SR01_b.2jm & 326 & 326 & 0.326 & 0.389836 \\
SR01_c.2jt & 314 & 314 & 0.314 & 0.375486 \\
SR01_d.2jW & 27 & 27 & 0.027 & 0.032287 \\
SR02_3j & 237 & 237 & 0.237 & 0.283408 \\
SR03_a.4j1- & 432 & 432 & 0.432 & 0.516592 \\
SR03_b.4j1 & 432 & 432 & 0.432 & 0.516592 \\
SR03_c.4jm & 50 & 50 & 0.05 & 0.0597908 \\
SR03_d.4jt & 232 & 232 & 0.232 & 0.277429 \\
SR03_e.4jW & 7 & 7 & 0.007 & 0.00837071 \\
SR04_5j & 269 & 269 & 0.269 & 0.321674 \\
SR05_a.6j1 & 119 & 119 & 0.119 & 0.142302 \\
SR05_b.6jm & 119 & 119 & 0.119 & 0.142302 \\
SR05_c.6jt & 82 & 82 & 0.082 & 0.0980569 \\
SR05_d.6jt+ & 139 & 139 & 0.139 & 0.166218 \\
\hline
\end{tabular}

These files start with some general information about the analysis and the analysed events. Note that the cross section error corresponds to $20 \%$ of the total cross section as specified in our CheckMATE input file for the $\tilde{g} \tilde{g}$ process.

After this, a list of all individual cutflow milestones/signal regions follows. For each of these, CheckMATE lists the sum of weights and sum of squared weights of all events that passed the corresponding cut(s) (Sum_W, Sum_W2), the relative efficiency times acceptance factor (Acc) as well as the expected number of events after normalising to the given total cross section and the luminosity of the respective analysis (N_Norm). In case of unweighted events, Sum_W and Sum_W2 corresponds to the number of Monte Carlo events in the respective region and this is true for the example above. However, if weighted events are used, they are properly taken into account and both Sum_W and Sum_W2 are required by CheckMATE's evaluation routines to properly calculate the statistical error in the upcoming evaluation step.

The cutflow information, similarly to all the files discussed in the previous paragraph, can be used e.g. for validation purposes. It is, however, currently not further processed by CheckMATE. The signal files, on the other hand, contain crucial information used for the subsequent evaluation step explained below.

Any output or warning/error messages generated during the analysis runs are stored in analysisstdout _analysisname.log files. If after a successful CheckMATE run these files are empty — as usually expected - they are removed automatically.

\subsubsection{Folder evaluation/}

The evaluation folder of our example run contains the following files:

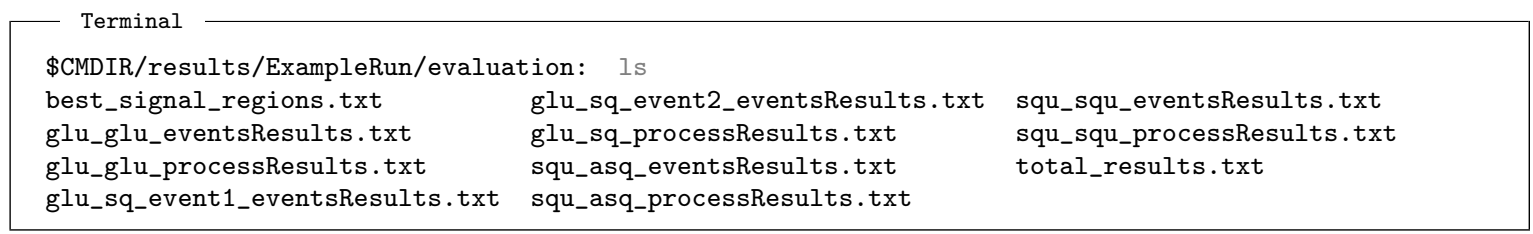

Files with name $X_{-}$eventResults.txt collect the results returned by all signal regions in all analyses for a given process X. By default, the data stored are the normalised number of predicted signal events (signal_normevents) and the total error on this number (signal_err_tot).

\begin{tabular}{|c|c|c|c|}
\hline analysis & sr & signal_normevents & signal_err_tot \\
\hline atlas_1308_1841 & SR01_8j50_a.0b & 0.0263608 & 0.00770593751115 \\
\hline $\begin{array}{l}\text { atlas_1308_1841 } \\
{[\ldots]}\end{array}$ & SR01_8j50_b.1b & 0.00838752 & 0.00358665176569 \\
\hline
\end{tabular}




$\begin{array}{llll}\text { atlas_1404_2500 } & \text { SR3Llow } & 0.0 & 0 \\ \text { atlas_1404_2500 } & \text { SR3b } & 0.0 & 0 \\ \text { atlas_1405_7875 } & \text { SR01_a.2jl } & 0.736622 & 0.150283717126 \\ \text { atlas_1405_7875 } & \text { SR01_b.2jm } & 0.389836 & 0.080901268657 \\ \text { [...] } & & & \\ \text { cms_sus_13_016 } & \text { SR1 } & 0.0 & 0\end{array}$

The error is defined as the quadratic sum of the statistical error, calculated internally from the size of the Monte Carlo sample, and the systematic error provided by the user. These individual error sources and additional columns can be requested by setting the correct options in the [Parameters] block in the CheckMATE setup file; see Section 3 .

During the evaluation phase, results from all individual processes are combined. First, results that correspond to the same process will be averaged by taking the corresponding weights properly into account. The statistical error is then calculated from the combined sum of weights and combined sum of squared weights. The statistical error for all signal regions with 0 Monte Carlo events at this stage is set to the corresponding statistical error of 1 Monte Carlo event ${ }^{19}$ The combined results of this procedure are stored in X_processResults.txt.

\begin{tabular}{|c|c|c|c|}
\hline $\begin{array}{l}{[\ldots]} \\
\text { atlas_1405_7875 } \\
{[\ldots]}\end{array}$ & $\mathrm{SR} 02 \_3 j$ & 10.9686 & 0.459030034105 \\
\hline
\end{tabular}

\begin{tabular}{llll|} 
glu_sq_event2_eventResults.txt & & \\
{$[\ldots]$} & & & \\
atlas_1405_7875 & SR02_3j & 11.2566 & 0.657620946569 \\
{$[\ldots]$} & & & \\
\hline
\end{tabular}

\begin{tabular}{llll|} 
glu_sq_processResults.txt & & \\
{$[\ldots]$} & & & \\
atlas_1405_7875 & SR02_3j & 11.0645722039 & 0.376429600571 \\
{$[\ldots]$} & & & \\
\hline
\end{tabular}

For processes with only one event file, the corresponding eventResults and processResults files are almost identical, except for the statistical error of signal regions with 0 events which is only set process-wise, not event-wise.

For the next step, results from different processes are added to determine the total expected number of signal events for each signal region. All errors are considered independent and hence added in quadrature. This is done for each signal region in each selected analysis separately. These results are then compared to the experimental limits using the chosen method, in our case the conservative $r$-limit since we did not specify anything else. The results for each analysis and each signal region are then stored in total_results.txt. Here, the standard columns are the number of experimentally observed, o, and expected Standard Model events, $\mathrm{b} \pm \mathrm{db}$, quoted by the experiments, the CheckMATE predicted number of signal events, $\mathrm{s}$, and the corresponding error, ds, the model independent $95 \%$ observed and expected limits, s95obs and s95exp, and the conservative $r$ value as defined in Eq. (1).

\footnotetext{
${ }^{19}$ This prescription ensures that Monte Carlo samples for processes with very large cross sections but with an insufficiently small number of events contribute with large statistical uncertainty to the final number, even if no signal event passed the cuts.
} 


\begin{tabular}{|c|c|c|c|c|c|c|c|c|c|c|}
\hline analysis & sr & 0 & $\mathrm{~b}$ & $\mathrm{db}$ & $s$ & $\mathrm{ds}$ & s95obs & s95exp & robscons & rexpcons \\
\hline atlas_1308_1841 & SR01_8j50_a.0b & 40.0 & 35.0 & 4.0 & 0.155 & 0.063 & 20.0 & 16.0 & 0.002543 & 0.003178 \\
\hline atlas_1308_1841 & SR01_8j50_b.1b & 44.0 & 40.0 & 10.0 & 0.021 & 0.050 & 23.0 & 23.0 & 0 & 0 \\
\hline atlas_1308_1841 & SR01_8j50_c.GE2b & 44.0 & 50.0 & 10.0 & 0.028 & 0.051 & 22.0 & 26.0 & 0 & 0 \\
\hline $\begin{array}{l}\text { atlas_1308_1841 } \\
{[\ldots]}\end{array}$ & SR02_9j50_a.Ob & 5.0 & 3.3 & 0.7 & 0.048 & 0.053 & 7.0 & 5.0 & 0 & 0 \\
\hline atlas_1405_7875 & SR01_d.2jW & 0.0 & 2.3 & 1.4 & 2.482 & 1.286 & 4.8 & 4.0 & 0.420647 & 0.504776 \\
\hline atlas_1405_7875 & SR02_3j & 7.0 & 5.0 & 1.2 & 19.58 & 1.222 & 8.2 & 6.4 & 2.242733 & 2.873502 \\
\hline $\begin{array}{l}\text { atlas_1405_7875 } \\
{[\ldots]}\end{array}$ & SR03_a.4jl- & 2169 . & .2120 & 110 & 22.54 & 0.282 & 270.0 & 240.0 & 00.07914 & 0.089036 \\
\hline cms_sus_13_016 & SR1 & 1.0 & 1.2 & 1.04 & 0.0 & 0.048 & 4.0 & 3.9 & 0 & 0 \\
\hline
\end{tabular}

(Note that we rounded the numbers in the table compared to the actual file content to fit the page width.)

In the last step of the evaluation procedure, CheckMATE will search for the signal region with the largest expected sensitivity. For the $r$-limits this corresponds to the signal region with the largest rexpcons. The results of the most sensitive signal region of each analysis is written in the file best_signal_regions.txt.

\begin{tabular}{|c|c|c|c|c|c|c|c|c|c|}
\hline analysis & sr & $\mathrm{b}$ & $\mathrm{db}$ & $\mathrm{s}$ & ds & s95obs & s95exp r & robscons & rexpcons \\
\hline atlas_1308_1841 & SR04_7j80_a.Ob & 11.0 & 2.2 & 0.21 & 0.0685 & 10.0 & $10.0 \quad 0$ & 0.010426 & 0.0104267 \\
\hline atlas_1308_2631 & SRA3 & 15.8 & 2.8 & 0.0 & 0.0492 & 9.0 & 10.2 & 0 & 0 \\
\hline atlas_1402_7029 & SR0taua20 & 0.29 & 0.18 & 0.0 & 0.0497 & 2.9 & 2.9 & 0 & 0 \\
\hline atlas_1403_4853 & L110 & 9.3 & 3.5 & 0.0 & 0.0497 & 9.0 & 9.4 & 0 & 0 \\
\hline atlas_1403_5222 & SR2B & 2.4 & 0.9 & 0.0 & 0.0497 & 3.4 & 4.5 & 0 & 0 \\
\hline atlas_1403_5294 & WWa_DF & 73.6 & 7.9 & 0.0 & 0.0497 & 20.3 & 22.5330 & 0 & 0 \\
\hline atlas_1403_5294_CR & CRmT2_top & 789. & 126.0 & 0.0 & 0.0497 & 253.0 & $250.0 \quad 0$ & 0 & 0 \\
\hline atlas_1404_2500 & SR1b & 4.7 & 2.1 & 0.0 & 0.0497 & 13.3 & 8.0 & 0 & 0 \\
\hline atlas_1405_7875 & SR02_3j & 5.0 & 1.2 & 19.5 & 0.7296 & 8.2 & 6.4 & 2.242733 & 2.8735021 \\
\hline atlas_1407_0583 & bCd_high1 & 11.0 & 1.5 & 0.0 & 0.0497 & 13.2 & 8.5 & 0 & 0 \\
\hline atlas_1407_0600 & SR017jA & 21.2 & 4.6 & 0.01 & 0.0492 & 13.9 & 13.8 & 0 & 0 \\
\hline atlas_1407_0608 & M3 & 1770 & 81.0 & 24.4 & 1.0220 & 195.0 & 190.00 & 0.116903 & 0.1199802 \\
\hline atlas_1411_1559 & SRTotal & 557. & 45.0 & 0.21 & 0.0880 & 70.0 & 91.0 & 0.000980 & 0.0007540 \\
\hline atlas_1501_07110 & SRmm-1 & 3.8 & 0.9 & 0.0 & 0.0497 & 7.9 & 6.0 & 0 & 0 \\
\hline atlas_1502_01518 & SR9 & 97.0 & 14.0 & 24.4 & 0.9640 & 58.0 & 36.0 & 0.394622 & 0.6357800 \\
\hline atlas_1503_03290 & $\mathrm{SR}-\mathrm{Z}$ & 10.6 & 3.2 & 0.0 & 0.0497 & 29.6 & 12.0 & 0 & 0 \\
\hline atlas_1506_08616 & SRinB & 14.1 & 2.8 & 0.0 & 0.0497 & 16.1 & 11.2 & 0 & 0 \\
\hline atlas_conf_2012_10 & el & 9.0 & 2.8 & 0.0 & 0.0142 & 9.9 & 9.3 & 0 & 0 \\
\hline atlas_conf_2012_14 & 4 & 380 & 73.40 & 4.47 & 0.3204 & 210.0 & $210.0 \quad 0$ & 0.018805 & 0.0188058 \\
\hline atlas_conf_2013_02 & emumu & 287 & 19.0 & 0.0 & 0.0318 & 58.2 & 49.7 & 0 & 0 \\
\hline atlas_conf_2013_02 & SR1 & 17.5 & 3.2 & 0.07 & 0.0511 & 10.0 & 10.6 & 0 & 0 \\
\hline atlas_conf_2013_03 & Higgs & 3450 & 180.0 & 0.0 & 0.0507 & 484.0 & $363.0 \quad 0$ & 0 & 0 \\
\hline atlas_conf_2013_03 & SR1Z & 1.3 & 1.0 & 0.0 & 0.0507 & 6.5 & 4.5 & 0 & 0 \\
\hline atlas_conf_2013_04 & SR_mT2_110_elmu & 4.4 & 2.0 & 0.0 & 0.0497 & 7.105 & 6.6990 & 0 & 0 \\
\hline atlas_conf_2013_06 & SROL7JA & 22.5 & 6.9 & 0.01 & 0.0492 & 15.3 & 14.6 & 0 & 0 \\
\hline atlas_conf_2013_06 & SoftLep1BHigh & 4.0 & 1.1 & 0.02 & 0.0508 & 7.9 & 6.3 & 0 & 0 \\
\hline atlas_conf_2013_08 & SR10F & 103. & 15.0 & 0.0 & 0.0497 & 24.0 & 31.0 & 0 & 0 \\
\hline atlas_conf_2014_01 & $\mathrm{SRa}$ & 53.0 & 10.0 & 0.0 & 0.0497 & 30.2 & 27.0 & 0 & 0 \\
\hline atlas_conf_2014_03 & emu & 4376 & 281.2 & 0.0 & 0.0497 & 1176.0 & $566.0 \quad 0$ & 0 & 0 \\
\hline atlas_conf_2014_05 & sig & 6000 & 3600 & 0.0 & 0.0497 & 6902.0 & 6717.00 & 0 & 0 \\
\hline atlas_conf_2015_00 & M1 & 578 & 48.41 & 0.04 & 0.0497 & 73.0 & 96.0 & 0 & 0 \\
\hline cms_1301_4698_WW & combined & 1000 & 60.0 & 0.0 & 0.00855 & 240.4 & 135.70 & 0 & 0 \\
\hline cms_1303_2985 & 23j_Ob_875 & 16.1 & 1.7 & 7.75 & 0.4298 & 18.545 & 10.11550 & 50.379938 & 0.6965528 \\
\hline cms_1405_7570 & Zjj_030 & 2136 & 859.0 & 0.0 & 0.0477 & 1378.8 & 1595.450 & & 0 \\
\hline cms_1408_3583 & 550 & 509 & 66.0 & 9.28 & 0.6481 & 129.0 & 123.00 & 0.063758 & 0.0668690 \\
\hline cms_1502_06031 & SR01_GE2jets_c.highMET & 12.8 & 4.3 & 0.0 & 0.0475 & 7.6 & 7.6 & 0 & 0 \\
\hline cms_1504_03198 & SR1 & 16.4 & 3.640 & 0.0 & 0.0482 & 12.9 & 11.4 & 0 & 0 \\
\hline cms_smp_12_006 & Oe & 487 & 40.0 & 0.0 & 0.0480 & 151.62 & $88.98 \quad 0$ & 0 & 0 \\
\hline cms_sus_12_019 & For_OF & 155. & 16.40 & 0.0 & 0.0475 & 31.8 & 31.8 & 0 & 0 \\
\hline cms_sus_13_016 & SR1 & 1.2 & 1.048 & 0.0 & 0.0477 & 4.0 & 3.9 & 0 & 0 \\
\hline
\end{tabular}

This file is helpful in getting a good overview of which analyses yield a non-vanishing $r$ value and hence show sensitivity to the tested model. In our example, one would expect that the most sensitive analyses 
are those targeting final states with a large jet multiplicity and missing transverse energy. Indeed, one can identify three such analyses with sizeable $r$-values: atlas_1405_7875 61], the zero lepton multijet search, the ATLAS monojet ${ }^{20}$ search atlas_1502_01518 62] and the CMS search cms_1303_2985 63] which uses the $\alpha_{T}$ variable to identify BSM events with a large hadronic activity.

CheckMATE then again chooses the most sensitive signal region among these. The corresponding observed result will be used to finally conclude whether the input can be considered excluded or not, i.e. in the case of the $r$-limit if robscons is larger than 1 . In the above example, the best signal region would be SR02_3j in the analysis atlas_1405_7875 which with the robscons value of about 2.2 excludes the tested model. This is exactly the result which was printed on screen at the end of our CheckMATE run.

With that, we have illustrated how CheckMATE can be used to test various BSM models with a range of input methods and which content can be found in all the produced output files. This knowledge should be sufficient for standard users to test their models of interest without much effort.

\section{Available Analyses}

A large number of ATLAS and CMS studies have been implemented covering a wide range of final state configurations. The vast majority of studies are dedicated searches for supersymmetry and generally require significant missing transverse momentum. They can be divided into four main categories.

- The powerful inclusive SUSY searches in final states with large jet multiplicities and large missing transverse momentum target the production of gluino and first/second generation squark pairs which subsequently cascade or directly decay into the lightest stable supersymmetric particle.

- Third generation searches are of particular interests due to the emerging little hierarchy problem and are sensitive to direct production of stop and sbottom pairs. In addition, searches for gluino induced stop and sbottom production (in cascade decays) are implemented in CheckMATE. The decays of the third generation sparticles yield top or bottom quarks in the final state.

- Electroweak searches target the direct production of electroweakinos and sleptons and generally focus on final states with at least two leptons (electrons or muons) or taus.

- Monojet (monophoton) searches which are sensitive to compressed spectra, simplified dark matter models, large extra dimensions, Higgs portals and other scenarios predicting a high momentum jet (photon) recoiling against missing transverse momentum.

A search for production of vector like top quarks as well as the rapidity gap signature in the vector boson fusion have also been implemented. Several analyses focused on SM measurements, like cross sections, are available as well. Searches for long-lived particles as well as the heavy Higgs boson searches are not included in the current CheckMATE version. Even though most of the currently implemented searches focus on SUSY, they can be applied to any non-supersymmetric BSM scenarios. In many cases some missing transverse momentum is expected, e.g. due to neutrinos from the SM gauge bosons decays which arise in a cascade decays of vector like quarks, for example. The analyses measuring SM cross sections typically also require rather small missing transverse momentum.

Most searches contain multiple signal regions, e.g. the stop pair production search with one isolated lepton, jets and missing transverse momentum [71 has 27 signal regions. It is, therefore, sensitive to a large class of mass hierarchies between the stop, chargino NLSP and neutralino LSP. Counting all signal regions of the implemented searches, 190 signal regions are employed just for the ATLAS searches at 8 TeV. We would like to caution users that different signal regions across different analyses are in many cases not statistically independent. The correlations can be particularly strong for the signal regions with similar final states and kinematic cuts. Any statistical combination should take this into account.

\footnotetext{
${ }^{20}$ Despite the description, this analysis allows for events with up to three hard jets in the final state and hence is also sensitive to our expected multijet signature.
} 
ATLAS $8 \mathrm{TeV}$ searches

\begin{tabular}{|c|c|c|c|c|c|c|}
\hline Name & Search & $\begin{array}{c}\sqrt{s} \\
{[\mathrm{TeV}]}\end{array}$ & $\begin{array}{c}\mathcal{L} \\
{\left[\mathrm{fb}^{-1}\right]}\end{array}$ & $N_{\mathrm{SR}}$ & Ref. & Cite \\
\hline atlas_1308_1841 & $\begin{array}{l}\text { New phenomena in final states with large jet mul- } \\
\text { tiplicities and } \mathbb{E}_{T}\end{array}$ & 8 & 20.3 & 19 & 64 & 65 \\
\hline atlas_1308_2631 & $\begin{array}{l}\text { Third-generation squark pair production in final } \\
\text { states with } \mathbb{E}_{T} \text { and two } b \text {-jets }\end{array}$ & 8 & 20.1 & 6 & 66 & \\
\hline atlas_1402_7029 & $\begin{array}{l}\text { Production of charginos and neutralinos in events } \\
\text { with three leptons and } \mathbb{E}_{T}\end{array}$ & 8 & 20.3 & 24 & 67 & \\
\hline atlas_1403_4853 & $\begin{array}{l}\text { Top-squark pair production in final states with two } \\
\text { leptons }\end{array}$ & 8 & 20.3 & 12 & 68 & \\
\hline atlas_1403_5222 & $\begin{array}{l}\text { Top squark pair production in events with a } Z \text { bo- } \\
\text { son, } b \text {-jets and } \mathbb{E}_{T}\end{array}$ & 8 & 20.3 & 5 & 69 & \\
\hline atlas_1404_2500 & $\begin{array}{l}\text { Supersymmetry in final states with jets and two } \\
\text { same-sign leptons or three leptons }\end{array}$ & 8 & 20.3 & 5 & 70 & \\
\hline atlas_1405_7875 & $\begin{array}{l}\text { Squarks and gluinos in final states with jets and } \\
\mathbb{E}_{T}\end{array}$ & 8 & 20.3 & 15 & 61 & 65 \\
\hline atlas_1407_0583 & $\begin{array}{l}\text { Top squark pair production in final states with one } \\
\text { isolated lepton, jets, and } \mathbb{E}_{T}\end{array}$ & 8 & 20.3 & 27 & 71 & \\
\hline atlas_1407_0608 & $\begin{array}{l}\text { Pair-produced third-generation squarks decaying } \\
\text { via charm quarks or in compressed supersymmetric } \\
\text { scenarios }\end{array}$ & 8 & 20.3 & 3 & 72 & \\
\hline atlas_1411_1559 & $\begin{array}{l}\text { Monophoton search with one energetic photon and } \\
\text { large } \mathbb{E}_{T}\end{array}$ & 8 & 20.3 & 1 & 73 & \\
\hline atlas_1501_07110 & $\begin{array}{l}\text { Search for direct pair production of a chargino and } \\
\text { a neutralino decaying to the } 125 \mathrm{GeV} \text { Higgs boson }\end{array}$ & 8 & 20.3 & 12 & 74 & \\
\hline atlas_1502_01518 & $\begin{array}{l}\text { New phenomena in final states with an energetic } \\
\text { jet and large } \mathbb{E}_{T}\end{array}$ & 8 & 20.3 & 9 & 62 & \\
\hline atlas_1503_03290 & $\begin{array}{l}\text { Supersymmetry in events containing a same- } \\
\text { flavour opposite-sign dilepton pair, jets, and large } \\
\mathbb{E}_{T}\end{array}$ & 8 & 20.3 & 1 & 75 & 65 \\
\hline atlas_1506_08616 & Pair production of third-generation squarks & 8 & 20.3 & 11 & 76 & \\
\hline atlas_conf_2012_104 & $\begin{array}{l}\text { Supersymmetry in final states with jets, } \mathbb{E}_{T} \text { and } \\
\text { one isolated lepton }\end{array}$ & 8 & 5.8 & 2 & 77 & \\
\hline atlas_conf_2012_147 & New phenomena in monojet plus $\mathbb{E}_{T}$ final states & 8 & 10 & 4 & 78 & \\
\hline atlas_conf_2013_024 & $\begin{array}{l}\text { Production of the top squark in the all-hadronic } t \bar{t} \\
\text { and } \mathbb{E}_{T} \text { final state }\end{array}$ & 8 & 20.5 & 3 & 79 & \\
\hline atlas_conf_2013_049 & $\begin{array}{l}\text { Direct-slepton and direct-chargino production in fi- } \\
\text { nal states with two opposite-sign leptons, } \mathbb{E}_{T} \text { and } \\
\text { no jets }\end{array}$ & 8 & 20.3 & 9 & 80 & \\
\hline atlas_conf_2013_061 & $\begin{array}{l}\text { Strong production of supersymmetric particles in } \\
\text { final states with } \mathbb{E}_{T} \text { and at least three } b \text {-jets }\end{array}$ & 8 & 20.1 & 9 & 81 & \\
\hline atlas_conf_2013_089 & $\begin{array}{l}\text { Strongly produced supersymmetric particles in de- } \\
\text { cays with two leptons }\end{array}$ & 8 & 20.3 & 12 & 82 & \\
\hline atlas_conf_2015_004 & $\begin{array}{l}\text { Invisibly decaying Higgs boson produced via vector } \\
\text { boson fusion }\end{array}$ & 8 & 20.3 & 1 & 83 & \\
\hline
\end{tabular}

Table 3: List of $8 \mathrm{TeV}$ ATLAS analyses which are available in the public alpha version of CheckMATE and which have been validated against published experimental results. The "Cite" column refers to an original paper by external authors who implemented a search and should be cited along with CheckMATE. 
CMS $8 \mathrm{TeV}$ searches

\begin{tabular}{|c|c|c|c|c|c|c|}
\hline Name & Search & $\begin{array}{c}\sqrt{s} \\
{[\mathrm{TeV}]}\end{array}$ & $\begin{array}{c}\mathcal{L} \\
{\left[\mathrm{fb}^{-1}\right]}\end{array}$ & $N_{\mathrm{SR}}$ & Ref. & Cite \\
\hline cms_1303_2985 & $\begin{array}{l}\text { Supersymmetry in hadronic final states with } \\
\text { missing transverse energy using the variables } \alpha_{T} \\
\text { and } b \text {-quark multiplicity }\end{array}$ & 8 & 11.7 & 59 & 63 & \\
\hline cms_1408_3583 & $\begin{array}{l}\text { Dark matter, extra dimensions, and unparticles } \\
\text { in monojet events }\end{array}$ & 8 & 19.7 & 7 & 84 & \\
\hline cms_1502_06031 & $\begin{array}{l}\text { Physics beyond the Standard Model in events } \\
\text { with two Leptons, jets, and } \mathbb{E}_{T}\end{array}$ & 8 & 19.4 & 6 & 85 & 65 \\
\hline cms_1504_03198 & $\begin{array}{l}\text { Production of dark matter in association with } \\
\text { top-quark pairs in the single-lepton final state }\end{array}$ & 8 & 19.7 & 1 & 86 & 87 \\
\hline cms_sus_13_016 & $\begin{array}{l}\text { New physics in events with same-sign dileptons } \\
\text { and jets }\end{array}$ & 8 & 19.5 & 1 & 88 & \\
\hline
\end{tabular}

Table 4: List of $8 \mathrm{TeV}$ CMS analyses which are available in the public alpha version of CheckMATE and which have been validated against published experimental results. The "Cite" column refers to an original paper by external authors who implemented a search.

ATLAS $13 \mathrm{TeV}$ searches

\begin{tabular}{|c|c|c|c|c|c|c|}
\hline Name & Search & $\begin{array}{c}\sqrt{s} \\
{[\mathrm{TeV}]}\end{array}$ & $\underset{\left[\mathrm{fb}^{-1}\right]}{\mathcal{L}}$ & $N_{\mathrm{SR}}$ & Ref. & Cite \\
\hline atlas_1602_09058 & $\begin{array}{l}\text { Supersymmetry in final states with jets } \\
\text { and } 2 \text { same sign leptons or } 3 \text { leptons }\end{array}$ & 13 & 3.2 & 4 & 89 & \\
\hline atlas_1604_01306 & $\begin{array}{l}\text { Search for new phenomena in events with a } \\
\text { photon and missing transverse momentum }\end{array}$ & 13 & 3.2 & 1 & 90 & \\
\hline atlas_1604_07773 & $\begin{array}{l}\text { Search for new phenomena in monojet } \\
\text { events }\end{array}$ & 13 & 3.2 & 13 & 91 & \\
\hline atlas_1605_03814 & $\begin{array}{l}\text { Squarks and gluinos in final states with } 2 \text { - } \\
6 \text { jets }+\mathbb{E}_{T}\end{array}$ & 13 & 3.2 & 7 & 92 & \\
\hline atlas_1605_04285 & $\begin{array}{l}\text { Gluino search in final states with an iso- } \\
\text { lated lepton }+ \text { jets }+\mathbb{E}_{T}\end{array}$ & 13 & 3.2 & 7 & 93 & \\
\hline atlas_1605_09318 & $\begin{array}{l}\text { Gluino pair productions in final states with } \\
b \text { jets and } \mathbb{E}_{T}\end{array}$ & 13 & 3.2 & 3 & 94 & \\
\hline atlas_1606_03903 & $\begin{array}{l}\text { Stop search in final states with } 1 \text { lepton, } \\
\text { jets and } \mathbb{E}_{T}\end{array}$ & 13 & 3.2 & 3 & 95 & \\
\hline atlas_conf_2015_082 & $\begin{array}{l}\text { Supersymmetry search in final states with } \\
\text { leptonic } \mathrm{Z}+\text { jets }+\mathscr{E}_{T}\end{array}$ & 13 & 3.2 & 1 & 96 & \\
\hline atlas_conf_2016_013 & $\begin{array}{l}\text { Vector like top quark production and } 4 \text { top } \\
\text { quark production in lepton plus jets final } \\
\text { state }\end{array}$ & 13 & 3.2 & 10 & 97 & \\
\hline atlas_conf_2016_050 & $\begin{array}{l}\text { Search for top squarks in final states with } \\
\text { one isolated lepton, jets, and } \mathbb{E}_{T}\end{array}$ & 13 & 13.2 & 5 & 98 & \\
\hline atlas_conf_2016_076 & $\begin{array}{l}\text { Search for direct top squark pair produc- } \\
\text { tion and dark matter production in final } \\
\text { states with two leptons }\end{array}$ & 13 & 13.3 & 6 & 99 & \\
\hline
\end{tabular}

Table 5: List of $13 \mathrm{TeV}$ ATLAS analyses which are available in the public alpha version of CheckMATE and which have been validated against published experimental results. 
CMS $13 \mathrm{TeV}$ searches

\begin{tabular}{|c|c|c|c|c|c|c|}
\hline Name & Search & $\begin{array}{c}\sqrt{s} \\
{[\mathrm{TeV}]}\end{array}$ & $\begin{array}{c}\mathcal{L} \\
{\left[\mathrm{fb}^{-1}\right]}\end{array}$ & $N_{\mathrm{SR}}$ & Ref. & Cite \\
\hline cms_pas_sus_15_011 & $\begin{array}{l}\text { Search for new physics in final states with } \\
\text { two opposite-sign same-flavor leptons, jets } \\
\text { and }\end{array}$ & 13 & 2.2 & 47 & 100 & \\
\hline
\end{tabular}

Table 6: List of $13 \mathrm{TeV}$ CMS analyses which are available in the public alpha version of CheckMATE and which have been validated against published experimental results.

ATLAS 14 TeV high luminosity studies

\begin{tabular}{|c|c|c|c|c|c|c|}
\hline Name & Search & $\begin{array}{c}\sqrt{s} \\
{[\mathrm{TeV}]}\end{array}$ & $\begin{array}{c}\mathcal{L} \\
{\left[\mathrm{fb}^{-1}\right]}\end{array}$ & $N_{\mathrm{SR}}$ & Ref. & Cite \\
\hline atlas_phys_2014_010_sq_hl & $\begin{array}{l}\text { Gluino and squark production in } \\
\text { final states with large jet multi- } \\
\text { plicities and } \mathbb{E}_{T} \text { and with no lep- } \\
\text { tons }\end{array}$ & 14 & 3000 & 10 & 101 & \\
\hline atlas_phys_2014_010_300 & $\begin{array}{l}\text { Gluino and squark production in } \\
\text { final states with large jet multi- } \\
\text { plicities and } \mathbb{E}_{T} \text { and with no lep- } \\
\text { tons }\end{array}$ & 14 & 300 & 10 & 101 & \\
\hline atlas_phys_2014_010_hl_31 & $\begin{array}{l}\text { Direct production of charginos } \\
\text { and neutralinos in final states } \\
\text { with three leptons and } \mathbb{E}_{T}\end{array}$ & 14 & 3000 & 1 & 101 & \\
\hline atlas_phys_2014_010_sbottom & $\begin{array}{l}\text { Direct production of sbottom } \\
\text { pairs in final states with } 2 b \text { jets } \\
\text { and } \mathbb{E}_{T}\end{array}$ & 14 & 3000 & 6 & 101 & \\
\hline atlas_phys_pub_013_011 & $\begin{array}{l}\text { Top squark pair production in fi- } \\
\text { nal states with }(b) \text { jets, } 0 \text { - } 1 \text { lep- } \\
\text { ton and } \mathbb{E}_{T}\end{array}$ & 14 & 3000 & 4 & 102 & \\
\hline
\end{tabular}

Table 7: List of official ATLAS $14 \mathrm{TeV}$ high luminosity analyses which are available in the public alpha version of CheckMATE and which have been validated against ATLAS MC results. 
All analyses listed in the above tables are fully validated against published cut-flows, distributions and/or exclusion limit plots from both experimental collaborations. The validation notes can be found on the official CheckMATE webpage. The analyses are grouped into ATLAS and CMS searches at the center of mass energies of 8 and $13 \mathrm{TeV}$ which are listed in Tables 3, 4, 5and 6. It is clear from the tables that ATLAS outnumbers CMS in the number of implemented analyses. This is due to the fact that the efficiencies of the final state particles have only been optimized and fully validated for the ATLAS detector. However, some CMS searches are also included, especially when there is no ATLAS equivalent or are of particular interest for our own phenomenological studies. The number of signal regions and the total integrated luminosity are given for each analysis in the corresponding tables. More details of the implemented searches can be found in the respective references provided in the tables.

In addition to the fully validated analyses, CheckMATE also includes some analyses which have not been completely validated. This can be, for example, due to insufficient information from the collaborations. Therefore some of these analyses are only partially validated and the full list can be found on the CheckMATE web page. If available, partial validation notes may also be provided and these often contain details regarding the outstanding issues that are still to be solved. Obviously, some caution is necessary when using these analyses for physics studies, especially if the particular signal regions of interest have not been completely tested. Altogether there are currently about 60 analyses available in CheckMATE and the list is expanding rapidly.

Current searches already push the exclusion limits of gluinos and first generation squarks well beyond the $\mathrm{TeV}$ scale. In the high luminosity phase, the limits will significantly improve and are of general interest. Therefore official ATLAS SUSY high-luminosity studies at a centre-of-mass energy of $14 \mathrm{TeV}$ for a total integrated luminosity of 300 and $3000 \mathrm{fb}^{-1}$ have now been included. Here, the high luminosity studies cover squark and gluino pair production, stop and sbottom pair production as well as chargino and neutralino production which are summarised in Table 7 .

\section{Performance Studies}

FRITZ allows for a significant gain in performance by bypassing the generation of HepMC or STDHEP event files, as well as not storing the detector level objects in a ROOT file. This new module interfaces Pythia, Delphes and the AnalysisHandler without the necessity to write and read information on the hard disk as described in detail in section 2. Here, we compare the performance between CheckMATE 1 and CheckMATE 2. In both frameworks, MC events are generated with Pythia 8 and the events are stored in a HepMC file for the CheckMATE 1 setup whereas for the CheckMATE 2 case the MC events are directly passed to the Delphes module with FRITZ. The computations are performed on an Intel(R) Xeon(R) CPU E5-2650 v2 @ $2.60 \mathrm{GHz}$ with $32 \mathrm{~GB}$ RAM. The performance has been quantified by generating 10000 gluino pairs at the LHC at the centre-of-mass energy of $8 \mathrm{TeV}$ with underlying event, initial and final state radiation and hadronisation switched on. The current version Pythia 8.2.1.9 was employed for this purpose. The events were passed to HepMC 2.0.6.09 and the resulting event file had a size of 2.3 GB. The truth level MC event generation and the write operation to hard disk took 255 seconds in total. The HepMC file was then passed to CheckMATE 1.2.2 and tested against a single inclusive supersymmetry search 61 and it took 85 seconds for CheckMATE 1 to process the HepMC file. Thus the total processing time was 340 seconds. This time was compared to the computing time of CheckMATE 2.0.0. The same process with the same sparticle spectrum and the identical Pythia settings were employed. After generating 10000 events on the fly, the total computational time was 245 seconds which clearly shows the improved performance.

For the next comparison, several instances were run at the same time. Here, it becomes clear that CheckMATE 2 has a big advantage over CheckMATE 1 since simultaneous read and write operations significantly affect the performance. Ten Pythia instances linked with CheckMATE 1 were simultaneously run. 20000 events were generated with the same settings as before. The event generation with Pythia took about 564 seconds. The truth level events were passed to CheckMATE 1 and writing the root files for the detector level objects took 1237 seconds on average. Contrary to CheckMATE 2 the root file must have been created before the analysis step could have been performed. The total processing time was 1802 seconds. We repeated the test with CheckMATE 2 and the average running time was 544 seconds. The CheckMATE 2 run is 
much faster since the reconstructed detector level objects are stored as ROOT objects which are immediately processed by the analysis module. It is evident that the bottleneck are the simultaneous write operations of ROOT files and this example clearly demonstrates the performance gain of CheckMATE 2 .

\section{Analysis Manager}

Along with the main CheckMATE program, several improvements have also been made to the AnalysisManager. Since these are only minor updates to the already existing program we refer the reader to original manual [27] and here we only list the changes.

\subsection{Prototyping New Analyses}

The most significant addition to the AnalysisManager is the improved support for analysis prototyping i.e. developing new LHC analyses. Analyses can now be added (using the usual interactive procedure) without accompanying signal region data (numbers of observed/expected events etc.) to allow a user to first simulate various SM backgrounds. The various background contributions can then be added for each defined signal region and for the development of new analyses we assume the expected background and observed data are equal.

After all the Standard Model contributions have been calculated, the AnalysisManager can then be rerun with a newly added option to edit the analysis information. Here a user should enter the total Standard Model background for all signal regions defined in the analysis. At this step the AnalysisManager will internally calculate $S 95$ limits so that new physics models can be quickly tested. 21

\subsection{Kinematical Variables}

To enable a quick and easy implementation of analyses, CheckMATE contains a large library of kinematical variables that are often used by the LHC collaborations. Firstly, CheckMATE is interfaced with the Delphes and ROOT libraries and thus a large number of kinematical variables such as transverse momentum, energy, pseudorapidity, boosts, etc. are immediately available. A list of Delphes objects and their methods can be found at https://cp3.irmp.ucl.ac.be/projects/delphes/wiki/WorkBook/RootTreeDescription. In addition, the TLorentzVector class from ROOT is included; see https://root.cern.ch/root/html/ TLorentzVector.html for more details.

CheckMATE also contains a number of LHC mass-reconstruction variables which are directly implemented or included from other libraries, e.g. implementation of $M_{T 2}$ and derivatives [10, 11] and MctLib [12, 13]. A full list of these kinematical variables is given in Table 8 and further details can be found in our doxygen documentation http://checkmate.hepforge.org/documentation/index.html. See Ref. [103, for a review of the kinematical variables proposed for mass reconstruction and BSM searches at the LHC.

\section{Summary}

We have introduced the second version of the program CheckMATE which greatly improves the ease and speed with which models can be tested against the latest LHC data compared to its predecessor. The major improvement in this release is the integration of Monte Carlo event generation that allows a user to go directly from a model defined in the UFO format to the LHC results. In addition, this integration significantly reduces the CPU load required to investigate models.

Further improvements are the inclusion of over 50 analyses which now cover the vast majority of LHC searches that include missing energy. Moreover, high luminosity studies at $14 \mathrm{TeV}$ are also included for the first time and these allow a user to understand the ultimate LHC reach for their model.

\footnotetext{
${ }^{21} \mathrm{By}$ default the AnalysisManager also now internally calculates all $S 95$ values rather than using those quoted by the experiments. The internally calculated values only show small differences compared to the numbers obtained the experiments. This approach ensures that the CheckMATE results are statistically consistent across all included analyses.
} 
Kinematical variables

\begin{tabular}{|c|c|c|}
\hline Name & Description/Example application & Ref. \\
\hline$M_{T}$ & Transverse Mass; reconstruction of $W$ mass in $W \rightarrow \ell \nu$ decay & $104-108$ \\
\hline$M_{T 2}$ & $\begin{array}{l}\text { Stransverse Mass; generalisation of } M_{T} \text { to events with more than one } \\
\text { invisible particle }\end{array}$ & 810 \\
\hline$M_{T 2}^{b \ell}$ & Asymmetric Stransverse Mass; suppression of SM top background & [10, 11 \\
\hline$M_{T 2}^{W}$ & $\begin{array}{l}\text { Asymmetric Stransverse Mass including } W \text { mass condition; suppression } \\
\text { of SM top background }\end{array}$ & {$[10,11$} \\
\hline$M_{C T}$ & Cotransverse Mass; invariant under contra-linear transverse boosts & 12 \\
\hline$M_{C T}$ corrected & Cotransverse Mass corrected; takes initial state radiation into account & 13 \\
\hline$M_{C T_{\perp}}$ and $M_{C T_{\|}}$ & Decomposed Cotransverse Mass & 14 \\
\hline$\alpha_{T}$ & Suppression of fake $\mathbb{E}_{T}$ in QCD events & 109,110 \\
\hline Razor & Mega-dijet kinematic variable without relying on $\mathbb{E}_{T}$ & $111-113$ \\
\hline 'Super'-Razor & $\begin{array}{l}\text { Improved Razor that more accurately determines production and centre- } \\
\text { of-mass frames }\end{array}$ & 16 \\
\hline Topness & Suppression of SM top background & 15 \\
\hline Aplanarity & Suppression of QCD events & 114 \\
\hline
\end{tabular}

Table 8: List of kinematical observables which are available in the public alpha version of CheckMATE.

For users who wish to include their own analyses or develop new LHC searches, the AnalysisManager also has a number of improvements to aid this process. As an example, backgrounds can be far more easily included and then used to test the reach of an analysis. In addition, the library of kinematical variables has grown significantly.

We should emphasise that the release of CheckMATE 2 is simply a snapshot of a continuously evolving program. New analyses will regularly be included in updated versions available on the website. Besides, many new developments are also planned for the CheckMATE including a fast parameter scanning technique that does not require Monte Carlo events, automatic merging of matrix elements containing different jet multiplicities and the inclusion of systematic correlations between signal regions to allow proper combinations of many different analyses.

\section{Acknowledgements}

We would like to thank all of the following people for their contribution to CheckMATE.

- Especially Liangliang Shang for contibuting analyses, performing validation studies and finding many bugs in CheckMATE.

- Junjie Cao, Jin Min Yang, Peiwen Wu, Jinmin Yang and Yang Zhang for contributing analyses to the CheckMATE database.

- Swasti Beswal, Anke Biekötter, Tim Keller and Jan Schütte-Engel for contributing analyses to the CheckMATE database.

- Sebastian Belkner for improving the statistical tools within CheckMATE.

- Florian Jetter for providing an updated muon resolution tuning.

- Daniel Antrim, Philip Bechtle, Jamie Boyd, Sascha Caron, Geraldine Conti, Carolina Deluca, Klaus Desch, Monica D'Onofrio, Till Eifert, Frank Filthaut, Eva Halkiadakis, Nicolai Hartman, Andreas Hoecker, Emma Kuwertz, Tommaso Lari, Zachary Marshall, Antoine Marzin, Federico Meloni, Alaettin Serhan Mete, Marija Vranjes Milosavljevic, Maurizio Pierini, Tina Potter, George Redlinger, Iacopo 
Vivarelli, Steven Worm, Frank Wuerthwein and Takashi Yamanaka for help with interpreting the experimental analyses.

The work has been supported by the BMBF grant 00160200. ND acknowledges partial support of the OCEVU Labex (ANR-11-LABX-0060), the A*MIDEX project (ANR-11-IDEX-0001-02) funded by the French Government programme "Investissements d'Avenir" and the German Research Foundation (DFG) through the Forschergruppe New Physics at the Large Hadron Collider (FOR 2239). The work of JSK was supported by IBS under the project code, IBS-R018-D1 and was partially supported by the MINECO, Spain, under contract FPA2013-44773-P; Consolider-Ingenio CPAN CSD2007-00042 and the Spanish MINECO Centro de excelencia Severo Ochoa Program under grant SEV-2012-0249. KR was supported by the National Science Centre (Poland) under Grant 2015/19/D/ST2/03136 and the Collaborative Research Center SFB676 of the DFG, "Particles, Strings, and the Early Universe".

\section{A. Installation Instructions}

CheckMATE uses a number of external programs and libraries ${ }^{22}$ Below we split these into two categories, those that are always required and those that can be optionally installed to extend the functionality of the program. We also note that a step by step interactive online version is available at,

$$
\text { http://checkmate.hepforge.org/tutorial/ver2/start.php }
$$

This tutorial is particularly useful if some of the below steps should be skipped, either because some of the programs have already been installed on the system or if some optional parts are not required. Also, the online tutorial will be continuously updated if the below installation routines especially of the required additional libraries change.

\section{A.1. Required Packages}

CheckMATE requires Python 2.7.X where $\mathrm{X}>3$ (note that at the current time, Python 3 is NOT supported), the data analysis package ROOT (v5.34.36 or later) [115] and the detector simulation Delphes (v3.3.3 or later) [1. If any of these packages are already installed, the respective sections of the tutorial can be skipped. The physics specific programs can be downloaded from the relevant project websites,

$$
\begin{gathered}
\text { https://root.cern.ch } \\
\text { https://cp3.irmp.ucl.ac.be/projects/delphes }
\end{gathered}
$$

We begin with the installation 23 of ROOT and we recommend that users do not install a binary version but rather compile the package from source into a specific installation directory. Here and in the following, we use [...] to denote the verbatim output created by the respective commands which we enter. These strongly depend on the system setup which is used. In the following we denote the installation directory by \$ROOT,

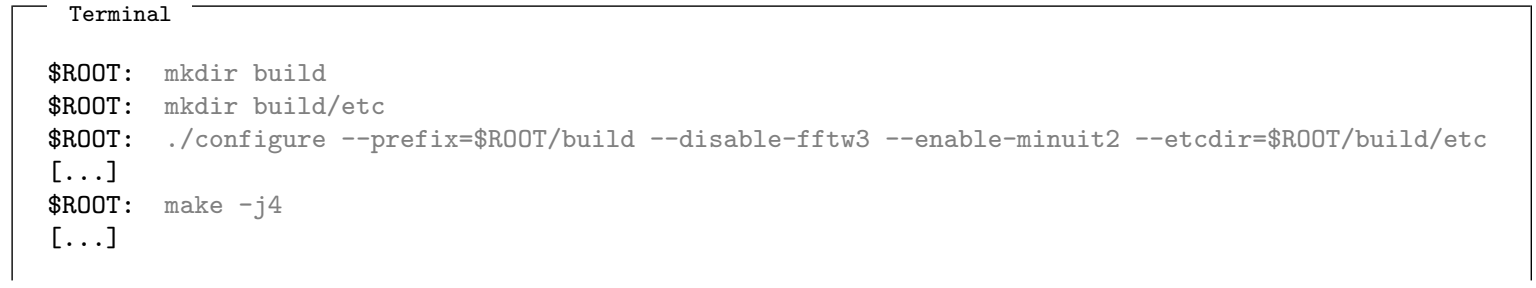

\footnotetext{
${ }^{22}$ This tutorial has been tested on a Linux machine running under Ubuntu 16.04. The same source files can be used for other operating systems, however some flags might change or some additional system libraries might be required. We refer to the documentation pages of the respective tools and the CheckMATE website if problems of that kind occur.

${ }^{23}$ Note that the $-\mathrm{j} 4$ flag which we use here improves the compilation speed due to paralellisation into four independent processes. The number can be changed depending on the number of accessible cores on the computer.
} 
In order to build the Delphes detector simulation framework in the installation directory denoted by \$DELPHES, we have to load the above compiled ROOT libraries

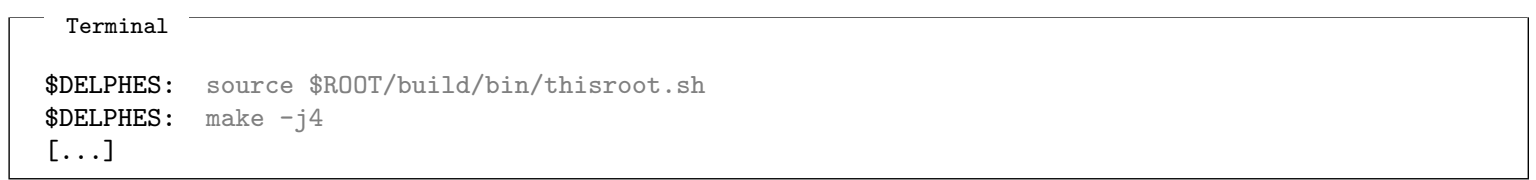

A promising sign of a successful installation is the presence of the file libDelphes.so within the Delphes directory.

\section{A.2. Optional Packages}

CheckMATE 2 now includes various options to generate events with Pythia 8 [7] and/or MadGraph5_aMC@NLO [6]. In addition, if the user wishes to have the possibility to store the generated events in the HepMC format [116, the corresponding library needs to be installed first. These programs can be downloaded from the relevant project websites,

http://hepmc.web.cern.ch/hepmc
http://home.thep.lu.se/ torbjorn/Pythia.html
https://launchpad.net/mg5amcnlo

We start with the installation of the optional HepMC library which is only required if the user wishes to save generated events in this format. Here the installation directory is denoted by \$HEPMC,



If the installation finished successfully the build directory should contain the required libraries and header files which are needed by Pythia 8,

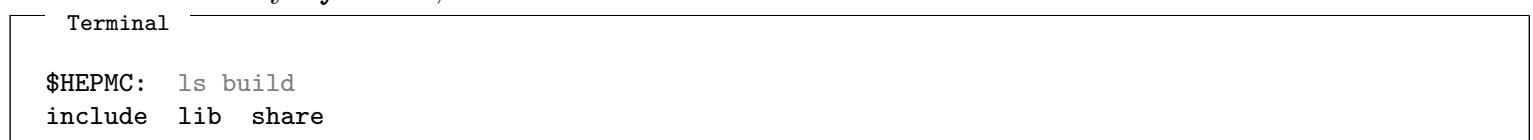

We can continue with the compilation and installation of the Pythia 8 event generator into the directory denoted by \$PYTHIA8. The command --with-hepmc2=\$HEPMC/build can be optionally removed if the HepMC library was not installed,

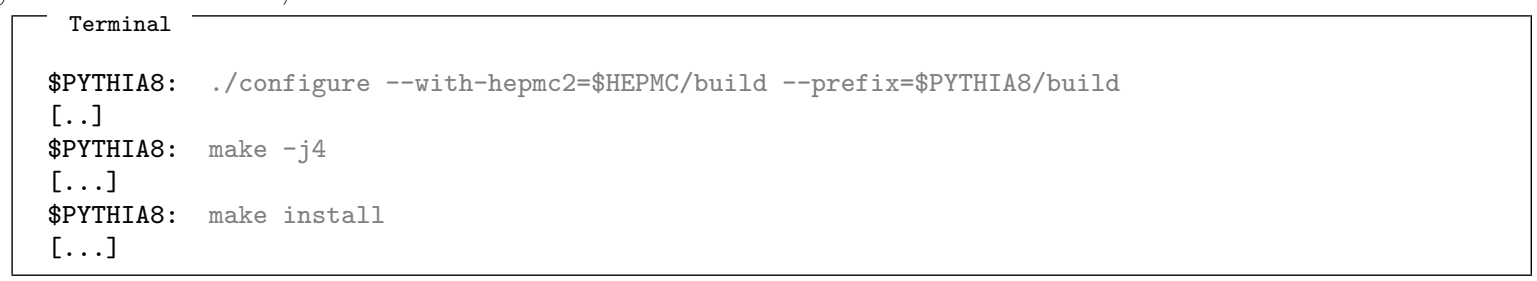

Again, a successful installation procedure should have filled the build directory with the necessary library files 


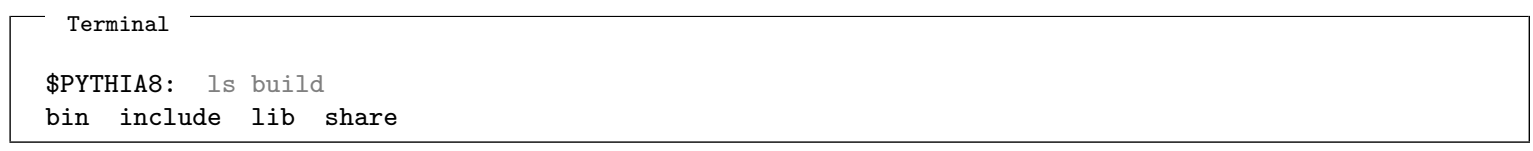

The final optional program CheckMATE can link to is MadGraph5_aMC@NLO and this only requires downloading and unzipping into the directory we denote by \$MADGRAPH.

\section{A.3. Installing CheckMATE}

With all required libraries being ready, we can finally compile the CheckMATE framework into the directory denoted by \$CMMAIN. In the following, any of the optional --with commands can be omitted if the relevant program has not been installed (note that Delphes and Root are required). The commands --with-gzipinc=\$GZIPINC and --with-gziplib=\$GZIPLIB are only required if Pythia 8 was compiled with gzip support. In this case, the same source header and library directories should be used.

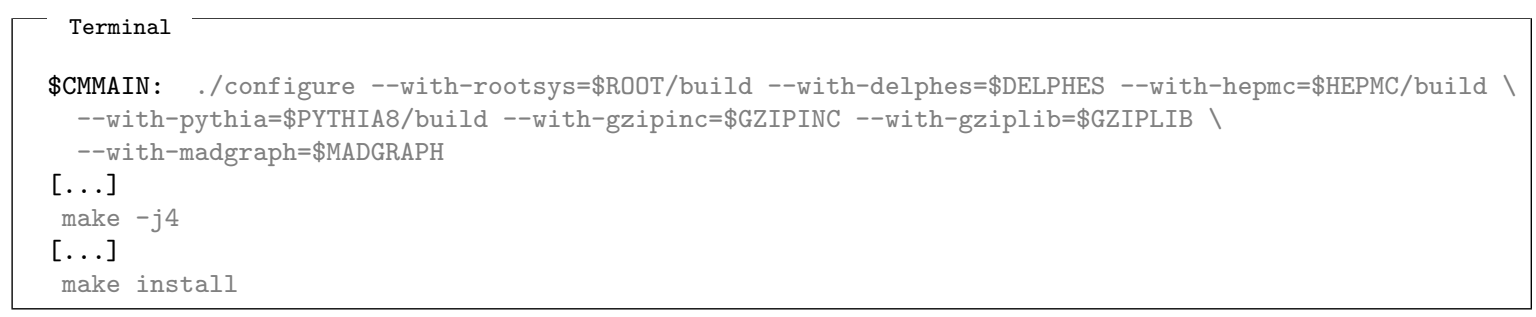

Let us finish this tutorial with a simple test run, using an example spectrum file which is provided with the CheckMATE package. It corresponds to a CMSSM scenario with $\tan \beta=10, m_{0}=100 \mathrm{GeV}, m_{1 / 2}=$ $250 \mathrm{GeV}, A_{0}=-100 \mathrm{GeV}$ and positive $\mu$. This results in a spectrum with all SUSY particles having mass in the range $100-600 \mathrm{GeV}$.



Such a SUSY scenario is so constrained by existing LHC searches that even a sample of only 100 Monte Carlo events is sufficient to exclude it within seconds.

\section{B. Statistical Analysis in CheckMATE}

A standard statistical problem which has to be solved in cut-based collider analyses is the following: What is the $p$-value of observing $N$ events in a certain bin if the Standard Model (= alternative hypothesis) predicts $B \pm \Delta B$ events and the new physics model (= null hypothesis) predicts $S \pm \Delta S$ events in addition to $B$ ? In this section we explain the exact procedure CheckMATE uses, based on the $\mathrm{CL}_{\mathrm{S}}$ prescription paired with a likelihood ratio discriminator [5]. For a more detailed description, we refer to Ref. 117].

\section{B.1. 1-bin Likelihood and Test Statistics}

If $S$ and $B$ were known with infinite precision, the likelihood of observing $N$ events in a bin where $S+B$ are theoretically expected would be given by the Poisson distribution

$$
\mathcal{L}(N \mid S)=\operatorname{Poiss}(N \mid S+B) \equiv \frac{(S+B)^{N}}{N !} e^{-(S+B)} .
$$


In reality, we do not know the background for certain but repeated evaluations would result in different values $B_{\text {unc. }}$. This parameter is distributed according to a probability density function $P\left(B\right.$ unc. $\left.\mid B, \Delta_{B}\right)$ with fixed $B, \Delta_{B}$. Even though in principle there can be arbitrary many independent $(\Delta B)_{i}$ from different error sources, CheckMATE only considers the combined background error $\Delta B$, cf. Section 4.3 . The algorithm described below can however be straightforwardly extended.

It is a common practice to redefine $B_{\text {unc. }}=B_{\text {unc. }}(\theta)$ in terms of a dimensionless nuisance parameter $\theta$. It is then $\theta$ which is distributed according to a density function $P(\theta \mid \tilde{\theta})$ with $\tilde{\theta}$ being the a priori most probable value. At the beginning, this value is trivially given via $B_{\text {unc. }}(\tilde{\theta})=B$, however along the calculation of $\mathrm{CL}_{\mathrm{S}}$, the value of $\tilde{\theta}$ will change as described below.

In CheckMATE, we always assume $B_{\text {unc. }}(\theta)=B \exp (\theta \Delta B / B)$ and $\theta$ to be Gaussianly distributed according to $P(\theta \mid \tilde{\theta}) \propto \exp \left(-(\theta-\tilde{\theta})^{2} / 2\right)$. With this choice of parameters, the a priori value for $\tilde{\theta}$ is 0 . For small $\Delta B / B$, this lognormal distribution will lead to a Gaussianly distributed $B_{\text {unc }}$ with mean $B$ and standard deviation $\Delta B$. However, for very large uncertainties it prevents $B(\theta)$ from turning to unphysical negative values.

Following the same approach for $S_{\text {unc }}(\theta)$ and assigning independent uncertainties to signal and background we get the following extended likelihood ${ }^{24}$

$$
\begin{aligned}
\mathcal{L}\left(N, \tilde{\theta}_{S}, \tilde{\theta}_{B} \mid \mu, \theta_{B}, \theta_{S}\right) & \equiv\left(\frac{1}{N !}\left[\lambda\left(\mu, \theta_{B}, \theta_{S}\right)\right]^{N} e^{-\lambda\left(\mu, \theta_{B}, \theta_{S}\right)}\right) \cdot\left(e^{-\left(\tilde{\theta}_{B}-\theta_{B}\right)^{2} / 2}\right) \cdot\left(e^{-\left(\tilde{\theta}_{S}-\theta_{S}\right)^{2} / 2}\right), \\
\lambda\left(\mu, \theta_{B}, \theta_{S}\right) & \equiv \mu S e^{\left(\frac{\Delta S}{S} \theta_{S}\right)}+B e^{\left(\frac{\Delta B}{B} \theta_{B}\right)} .
\end{aligned}
$$

Note that we have introduced the signal strength modifier $\mu$, which will prove more convenient in distinguishing signal and background hypotheses for varying $S_{\mathrm{unc}}\left(\theta_{S}\right)$.

There are different approaches to incorporate the unknown nuisance parameters $\theta_{B}, \theta_{S}$ into the test statistics. CheckMATE uses the Profile Likelihood Ratio defined as

$$
q_{\mu}\left(N, \tilde{\theta}_{S}, \tilde{\theta}_{B}\right) \equiv-2 \log \left(\frac{\mathcal{L}\left(N, \tilde{\theta}_{S}, \tilde{\theta}_{B} \mid \mu, \hat{\theta}_{S}^{\mu}, \hat{\theta}_{B}^{\mu}\right)}{\mathcal{L}\left(N, \tilde{\theta}_{S}, \tilde{\theta}_{B} \mid \hat{\mu}, \hat{\theta}_{S}, \hat{\theta}_{B}\right)}\right)
$$

Here, $\hat{\mu} \in[0, \mu]^{25} \hat{\theta}_{S}$ and $\hat{\theta}_{B}$ is the combination of all three parameters which globally maximises $\mathcal{L}$, whereas $\hat{\theta}_{S}^{\mu}, \hat{\theta}_{B}^{\mu}$ are the values which maximise $\mathcal{L}(\mu)$ for fixed $\mu . q_{\mu}\left(N, \tilde{\theta}_{S}, \tilde{\theta}_{B}\right)$ becomes larger for less compatibility of observation and null hypothesis. According to Wilks' theorem [118, the maximum likelihood ratio approaches a $\chi^{2}$-distribution for large event rates.

Note that even for our rather simple statistical setup, the numerator cannot be evaluated analytically. As such, CheckMATE uses the numerical scipy.optimize.root routine to find the roots of the first derivatives in order to the evaluate the test statistics.

\section{B.2. Confidence Levels and p-values}

With the test statistics of Eq. (5), we can determine the $p$-value of the signal hypothesis $S+B$ after observing $N$ as follows. If we repeated the experiment infinitely many times we would expect to observe different values $N^{\prime}$ each time such an experiment is performed due to the statistical nature of the underlying physics. Also, our determination of the distributions for $B_{\text {unc }}\left(\theta_{B}\right), S_{\text {unc }}\left(\theta_{S}\right)$ would have resulted in different values for the expectation values $\tilde{\theta}_{B}, \tilde{\theta}_{S}$ which we a priori assumed to be 0 . The values which we would expect depend on the underlying hypothesis we assume.

If the signal hypothesis $\mu=1$ was correct, according to the likelihood in Eq. (3) the most compatible values for the nuisance parameters $\theta_{S}, \theta_{B}$ after the observation of $N$ would be their best fit values $\hat{\theta}_{S}^{\mu=1}$ and

\footnotetext{
${ }^{24}$ Note that we can safely ignore normalisation factors for the Gaussian distributions as they will not contribute to the likelihood ratio.

${ }^{25}$ The lower limit $\hat{\mu} \geq 0$ leads to a one-sided limit on $S$ while the upper limit $\hat{\mu} \leq \mu$ ensures that the test statistics is 0 if the global best fit would prefer an even larger signal than the one tested. In other words, we claim perfect compatibility of signal hypothesis and observation also if a larger signal would fit the observation better.
} 
$\hat{\theta}_{B}^{\mu=1}$. If we thus a posteriori assume that these were the true expected values of $\tilde{\theta}_{S}, \tilde{\theta}_{B}$ and we hypothetically redid the experiment we would expect a random value of $\tilde{\theta}_{B}$ according to a Gaussian distribution with expectation value $\hat{\theta}_{B}^{\mu=1}$, analogously for $\tilde{\theta}_{S}$. Also, the number of observed events $N^{\prime}$ should be Poisson distributed with the expectation value $\lambda\left(\mu=1, \hat{\theta}_{S}^{\mu=1}, \hat{\theta}_{B}^{\mu=1}\right)$.

For a $p$-value we are interested in the fraction of these hypothetical experiments which would perform in a test statistics at least as bad as the observed one. We call this value for the signal hypothesis $\mathrm{CL}_{S+B}$ and formulate it analytically as follows:

$$
\begin{aligned}
\mathrm{CL}_{S+B} \equiv \sum_{N^{\prime}=0}^{\infty} \int_{-\infty}^{\infty} \mathrm{d} \tilde{\theta}_{S}^{\prime} \int_{-\infty}^{\infty} \mathrm{d} \tilde{\theta}_{B}^{\prime} \Theta\left(q_{\mu=1}\left(N^{\prime}, \tilde{\theta}_{S}^{\prime}, \tilde{\theta}_{B}^{\prime}\right)-q_{\mu=1}\left(N, \tilde{\theta}_{S}, \tilde{\theta}_{B}\right)\right) \times \\
\operatorname{Poiss}\left(N^{\prime} \mid \lambda\left(\mu=1, \hat{\theta}_{S}^{\mu=1}, \hat{\theta}_{B}^{\mu=1}\right) \cdot \operatorname{Gauss}\left(\tilde{\theta}_{S}^{\prime} \mid \hat{\theta}_{S}^{\mu=1}\right) \cdot \operatorname{Gauss}\left(\tilde{\theta}_{B}^{\prime} \mid \hat{\theta}_{B}^{\mu=1}\right),\right.
\end{aligned}
$$

where we define $\Theta(x)=1$ for $x \geq 0$ and 0 else.

This approach however has a peculiar property: If, for example, $N$ happens to be much smaller than $B-2 \Delta B$, which statistically can happen in a small fraction of experiments even if $B$ describes Nature accurately, $\mathrm{CL}_{S+B}$ will always turn out to be small, regardless of $S$. Therefore, the above interpretation will always claim a tension with the signal hypothesis and could even conclude that a model with $S \ll \Delta B$ is excluded. One should be worried in this case as it intuitively sounds incorrect to conclude anything about a signal which is much smaller than the systematic uncertainty of the experiment.

A commonly used approach to avoid such a false exclusion is to, in addition to $\mathrm{CL}_{S+B}$, determine the $p$-value for the observation to be compatible with the background-only hypothesis. For that purpose, we simply set $\mu=0$ in our above explanation and thus evaluate

$$
\begin{aligned}
1-\mathrm{CL}_{B}=\sum_{N^{\prime}=0}^{\infty} \int_{-\infty}^{\infty} \mathrm{d} \tilde{\theta}_{S}^{\prime} \int_{-\infty}^{\infty} \mathrm{d} \tilde{\theta}_{B}^{\prime} \Theta\left(q_{\mu=1}\left(N^{\prime}, \tilde{\theta}_{S}^{\prime}, \tilde{\theta}_{B}^{\prime}\right)-q_{\mu=1}\left(N, \tilde{\theta}_{S}, \tilde{\theta}_{B}\right)\right) \times \\
\quad \operatorname{Poiss}\left(N^{\prime} \mid \lambda\left(\mu=0, \hat{\theta}_{S}^{\mu=0}, \hat{\theta}_{B}^{\mu=0}\right) \cdot \operatorname{Gauss}\left(\tilde{\theta}_{S}^{\prime} \mid \hat{\theta}_{S}^{\mu=0}\right) \cdot \operatorname{Gauss}\left(\tilde{\theta}_{B}^{\prime} \mid \hat{\theta}_{B}^{\mu=0}\right) .\right.
\end{aligned}
$$

Note that it is a common misconception to evaluate the test statistics of $1-\mathrm{CL}_{B}$ with $q_{\mu=0}$ instead of $q_{\mu=1}$. However, through the entire limit setting procedure we are testing the signal hypothesis and at this stage we are estimating what the result of this test would be if the background hypothesis was true. This is why we use $\mu=0$ for the expectation values of $N^{\prime}, \tilde{\theta}_{S}$ and $\tilde{\theta}_{B}$ but still need to evaluate the test statistics for the signal hypothesis $\mu=1$.

In the $\mathrm{CL}_{S}$ prescription, the confidence in the signal hypothesis is calculated by the ratio

$$
\mathrm{CL}_{S} \equiv \frac{\mathrm{CL}_{S+B}}{1-\mathrm{CL}_{B}}
$$

and we interpret this value as the $p$-value for our signal. Consequently, we exclude a signal model if it produces a too small $\mathrm{CL}_{S}$ value. If an experiment shows a perfect agreement with the Standard Model prediction, $1-\mathrm{CL}_{B}$ equals 0.5 and therefore $\mathrm{CL}_{S}$ is very close to the true $p$-value $\mathrm{CL}_{S+B}$. For experiments which are in tension with the background-only hypothesis, $1-\mathrm{CL}_{B}$ becomes smaller, $\mathrm{CL}_{S}$ increases and thus the limit weakens. $\mathrm{CL}_{S} \mathrm{CL}_{S}$ therefore sets a conservative limit in the presence of under-fluctuations in the data.

CheckMATE evaluates the integrals in Eqs. (6) and (7) numerically by generating tuples of random numbers $N^{\prime}, \tilde{\theta}_{B}, \tilde{\theta}_{S}$ according to the respective Poisson or Gaussian distribution and counting the relative amount of tuples which yield a value of $q_{\mu=1}$ smaller than $q_{\mu=1}(N, 0,0)$.

\section{B.3. Model Independent Limits S95}

In the main text, we explained how CheckMATE usually does not calculate $\mathrm{CL}_{S}$ in each run but normally calculates $r=(S-1.64 \cdot \Delta S) / S 95$ with the model independent upper $95 \%$ confidence limit $S 95$. As the value for $\Delta S$ is not known in that case, it is set to 0 for the following evaluation. CheckMATE uses 
simplified versions of the above described formulae with all terms depending on $\theta_{S}$ removed and using $\lambda\left(\mu, \theta_{B}\right) \equiv \mu S+B \exp \left(\frac{\Delta B}{B} \theta_{B}\right)$.

For the observed limit, S95_obs, one simply needs to find the value of $S$ which yields $\mathrm{CL}_{S}=0.05$. CheckMATE uses the Pegasus regula-falsi method for this purpose [119. For the expected limit, S95_exp, CheckMATE determines the limit if we observed what we derived as the true background values from our observation, see Section B.2 above. That means we set $N=\lambda\left(\mu=0, \hat{\theta}_{B}^{\mu=0}\right)$ and the a priori $\tilde{\theta}_{B}$ to $\hat{\theta}_{B}^{\mu=0}$ and follow the same prescription as before.

\section{B.4. Likelihood}

As mentioned in Section 3. CheckMATE is capable of returning a combined likelihood summed over all bins. Here, it calculates the test statistics as in Eq. (5) and sums the result over all bins. For this purpose, we remove the restriction $\hat{\mu} \in[0, \mu]$ from Eq. (5) as we are not trying to perform a one-sided signal test. This calculation can be done for all bins and the sum of all likelihoods over all considered signal regions is returned by CheckMATE.

We note that a user should exercise care with the likelihood calculation since it can only be applied to orthogonal signal regions and some of the analyses included in CheckMATE do not fulfil this condition. If the user wishes to include such analyses, the likelihood sum should be calculated manually by selecting the result of the relevant orthogonal signal regions.

\section{Tuning}

In this appendix we provide details of the new CheckMATE tunings for lepton efficiencies and $b$-tagging. In the case of leptons we use recent ATLAS parametrisations updated during the 8 and 13 TeV runs. The new lepton energy resolutions have been implemented in the Delphes cards for 13 and 14 TeV analyses. The efficiency is evaluated internally by the respective AnalysisHandlers.

\section{C.1. Electrons}

Electron identification in the ATLAS detector is based on the multivariate analysis (MVA) that simultaneously evaluates several properties of the electron candidates. Three levels of identification are implemented in CheckMATE that correspond to the typical ATLAS operating points: loose, medium and tight. The efficiency depends on the transverse energy of the electron candidate, $E_{T}$, and to a lesser extent on the rapidity [120. The current implementation in the AnalysisHandler takes into account only the former. The following functions are used for the $13 \mathrm{TeV}$ setup:

$$
\begin{aligned}
& \epsilon_{\mathrm{id}}^{\text {loose }}=0.976-0.0614 \cdot \exp \left(1-\frac{E_{T}}{29.1}\right), \\
& \epsilon_{\mathrm{id}}^{\text {medium }}=0.937-0.109 \cdot \exp \left(1-\frac{E_{T}}{21.0}\right), \\
& \epsilon_{\mathrm{id}}^{\text {tight }}=0.8885-0.138 \cdot \exp \left(1-\frac{E_{T}}{27.5}\right),
\end{aligned}
$$

where $E_{T}$ is the transverse energy of the candidate in $\mathrm{GeV}$. The parameters in the above parametrisation were obtained from the fit to the efficiency plots reported by ATLAS [120, 121]. Figure 2 shows the electron reconstruction and identification efficiency reported by ATLAS and obtained with CheckMATE for tight, medium and loose electrons as a function of electron candidate transverse energy.

For the parametrisation of electron energy resolution we follow Ref. 122. The functions describing smearing of energy and momenta are the following:

$$
\begin{aligned}
& \sigma(\mathrm{GeV})=0.3 \oplus 0.10 \times \sqrt{E(\mathrm{GeV})} \oplus 0.010 \times E(\mathrm{GeV}) \quad \text { for } \quad|\eta|<1.4, \\
& \sigma(\mathrm{GeV})=0.3 \oplus 0.15 \times \sqrt{E(\mathrm{GeV})} \oplus 0.015 \times E(\mathrm{GeV}) \quad \text { for } \quad 1.4<|\eta|<2.47 .
\end{aligned}
$$

Here, $a \oplus b \equiv \sqrt{a^{2}+b^{2}}$. The functions are implemented in the delphes_skimmed_ATLAS_13TeV.tcl and delphes_skimmed_ATLAS_14TeV.tcl cards. 




Figure 2: Comparison of the combined electron reconstruction and identification efficiency reported by ATLAS (full markers; see Figure 14 of Ref. 120) and obtained with CheckMATE (empty markers) for tight (black triangle), medium (red square) and loose (blue circle) electrons as a function of electron candidate transverse energy and in the full pseudorapidity range, $|\eta|<2.47$.

\section{C.2. Muons}

Muon reconstruction and identification efficiency shows generally an excellent performance across different rapidities and energy ranges 123 . In CheckMATE the efficiency is about 0.99 for loose muons and 0.97 for tight muons, except for muon candidates with $|\eta|<0.1$ where it is set to 0.6 . The dependence on $p_{T}$, unlike for the electrons, is negligible.

The combined muons correspond to muons observed in the inner detector (ID) and in the muon spectrometer (MS). The resolution formula combines the information from both systems and is given by [124]:

$$
\begin{aligned}
& \sigma_{\mathrm{ID}}=p_{T} \times \sqrt{a_{1}^{2}+\left(a_{2} \times p_{T}\right)^{2}}, \\
& \sigma_{\mathrm{MS}}=p_{T} \times \sqrt{\left(\frac{b_{0}}{p_{T}}\right)^{2}+b_{1}^{2}+\left(b_{2} \times p_{T}\right)^{2}}, \\
& \sigma_{\mathrm{CB}}=\frac{\sigma_{\mathrm{ID}} \times \sigma_{\mathrm{MS}}}{\sqrt{\sigma_{\mathrm{ID}}^{2}+\sigma_{\mathrm{MS}}^{2}}},
\end{aligned}
$$

where $p_{T}$ is the truth transverse momentum in GeV. The coefficients $b_{0}, b_{1}, b_{2}$ are specified in Table 9 and are the same for 13 and $14 \mathrm{TeV}$ analyses. In the same table we also provide coefficients $a_{1}$ and $a_{2}$ for the $13 \mathrm{TeV}$ setup. For the HL option the $a_{1}$ and $a_{2}$ coefficients are specified in 15 separate regions in rapidity taking into account planned upgrades to the inner detector. The full list can be found in Ref. 124.

\section{C.3. B-Tagger}

The quality of algorithms that try to filter jets containing $b$-quarks from others is determined by two main quantities. The signal efficiency describes the probability to assign a tag to a jet that actually contains a $b$-quark, whereas the background efficiency is a measure for the relative amount of jets that are tagged even though they do not have any bottom quark content. Since the background efficiency is usually small, it is common to use the inverse value, called rejection, for illustrative purposes. Also, one usually distinguishes 


\begin{tabular}{llllll}
\hline \hline & $a_{1}$ & $a_{2}$ & $b_{0}$ & $b_{1}$ & $b_{2}$ \\
\hline$|\eta|<1.05$ & 0.01607 & 0.000307 & 0.24 & 0.02676 & 0.00012 \\
$|\eta|>1.05$ & 0.03000 & 0.000387 & 0.00 & 0.03880 & 0.00016 \\
\hline \hline
\end{tabular}

Table 9: The muon resolution coefficients $a_{1}, a_{2}, b_{0}, b_{1}, b_{2}$ from Eqs. 14 and 15 for the 13 TeV ATLAS setup.

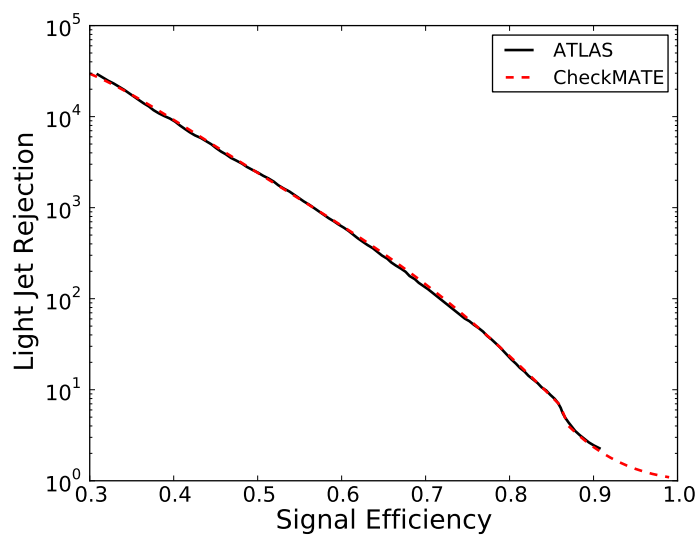

(a) Rejection curve for jets containing light quarks only.

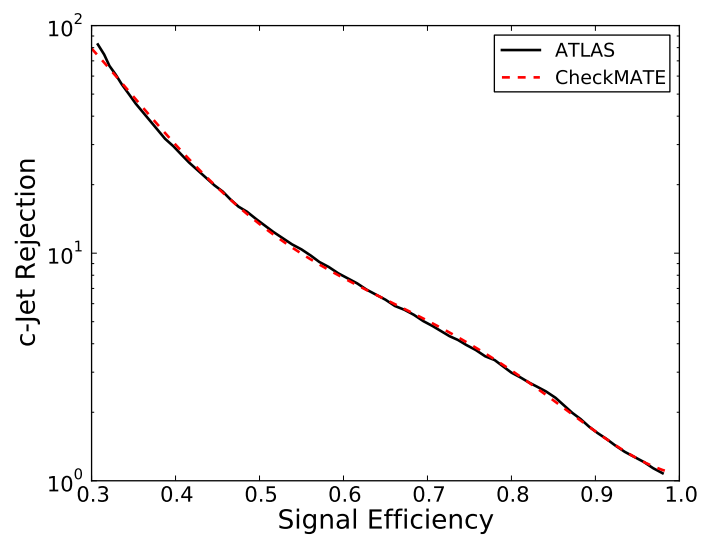

(b) Rejection curve for jets with charm content.

Figure 3: Receiver Operation Characteristic curves for a dependence of the background rejection for jets with different quark contents on a chosen signal efficiency working point of the $b$-tagger [125].

between rejections against jets with charm content and other jets that only contain light quarks, as the first are harder to distinguish from the signal.

Since the rejection gets weaker with increasing signal efficiency, one has to find a balance between signal quantity and signal purity, which depends crucially on the details of the respective analysis. For this purpose, one uses the ROC (Receiver Operation Characteristic) curve that describes the relation between these two quantities.

\section{C.3.1. $8 \mathrm{TeV}$}

We show the ROC curves for light-jet and $c$-jet rejection separately in Figure 3 and internally parametrise these as follows:

$$
\begin{aligned}
\log _{10}\left[\bar{r}_{\text {light }}\left(\bar{\epsilon}_{\mathrm{S}}\right)\right] & = \begin{cases}52.8 \cdot\left(\bar{\epsilon}_{\mathrm{S}}-4.045 \cdot \bar{\epsilon}_{\mathrm{S}}^{2}+7.17 \cdot \bar{\epsilon}_{\mathrm{S}}^{3}-6.14 \cdot \bar{\epsilon}_{\mathrm{S}}^{4}+2.01 \cdot \bar{\epsilon}_{\mathrm{S}}^{5}\right) & \text { if } \bar{\epsilon}_{\mathrm{S}}<0.87, \\
-75.4 \cdot\left(\bar{\epsilon}_{\mathrm{S}}-1.07\right)^{3} & \text { if } \bar{\epsilon}_{\mathrm{S}} \geq 0.87,\end{cases} \\
\log _{10}\left[\bar{r}_{\mathrm{c}}\left(\bar{\epsilon}_{\mathrm{S}}\right)\right] & =29.3 \cdot\left(\bar{\epsilon}_{\mathrm{S}}-4.572 \cdot \bar{\epsilon}_{\mathrm{S}}^{2}+8.496 \cdot \bar{\epsilon}_{\mathrm{S}}^{3}-7.253 \cdot \bar{\epsilon}_{\mathrm{S}}^{4}+2.33 \cdot \bar{\epsilon}_{\mathrm{S}}^{5}\right),
\end{aligned}
$$

where $\bar{\epsilon}_{\mathrm{S}}$ is signal efficiency and $\bar{r}_{\text {light/c }}$ background rejection for light and $c$-jets, respectively.

Given a particular working point on the ROC curve, i.e. a specific chosen signal efficiency, $\bar{\epsilon}_{\mathrm{S}}$, and the corresponding background rejections, $\bar{r}_{\text {light/c }}$, the actual tagging probabilities depend on the transverse momentum of the considered object. These have been measured individually for signal, light-quark and $D^{*}$ mesor ${ }^{26}$ jets and we show the results in Figure 4.

\footnotetext{
${ }^{26}$ The tagging probability for jets containing $D^{*}$ mesons is roughly 2 times higher than for other $c$-mesons. Using the cutflows from various analyses we have tuned this parameter to 0.4 to be in agreement with the ATLAS results.
} 


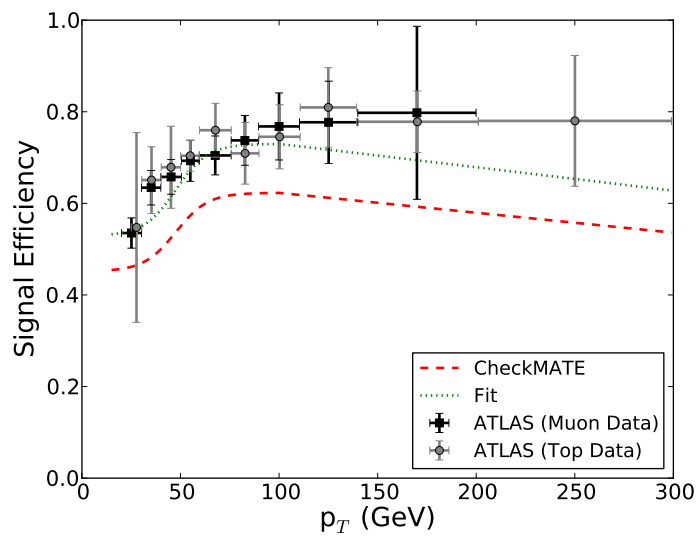

(a) Signal efficiencies for $b$-tagging, determined by combining the information from two different search channels for $\bar{\epsilon}_{\mathrm{S}}=0.7[125,126]$.

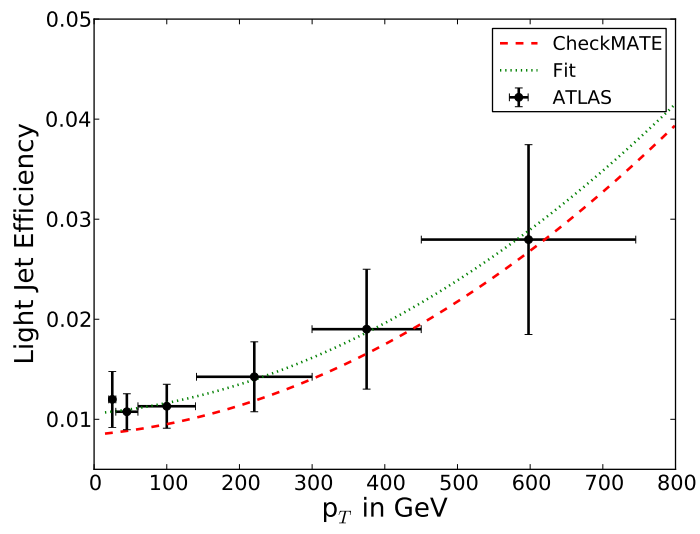

(c) b-tagging efficiency of jets containing light quarks for $\bar{\epsilon}_{\mathrm{S}}=0.7$ and $|\eta|<1.3$ 127.

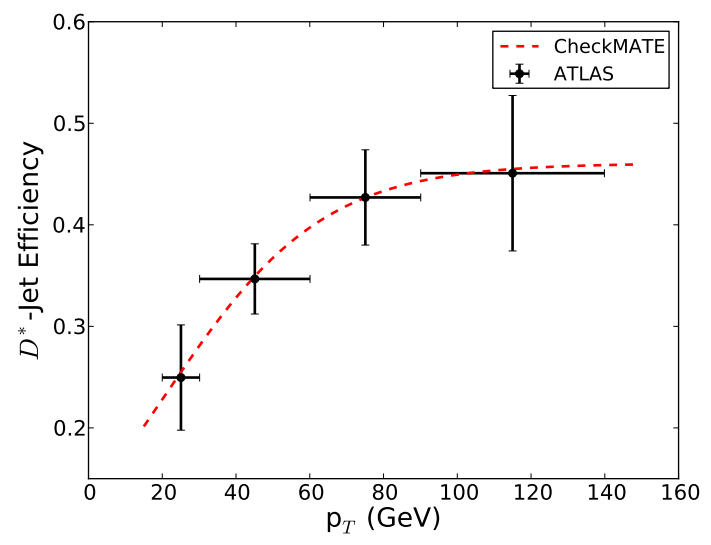

(b) $b$-tagging efficiency of jets containing $D^{*}$ mesons for $\bar{\epsilon}_{\mathrm{S}}=0.7$ [126]. The inclusive efficiency for $c$-jets is assumed to be $40 \%$ of this $D^{*}$ efficiency.

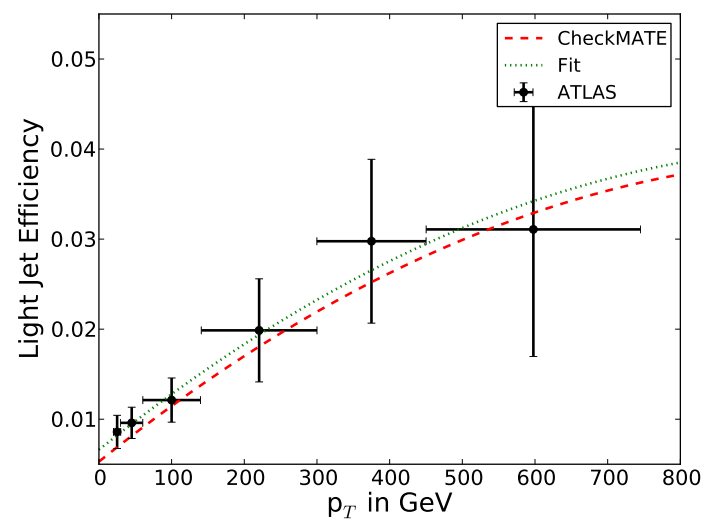

(d) Same as (c) for $1.3 \leq|\eta|<2.5$.

Figure 4: Dependence on the signal and light-jet / $c$-jet background efficiencies for $b$-tagging on the transverse momentum of the jet candidate.

For the signal efficiency, we use two different data sets as they have different sensitivities at low and high energies; see Figure 4a In order to agree with the cutflows of various analyses that require $b$-tagging, a reduction in the overall normalisation by $15 \%$ has been applied. In addition, a significant decrease of the signal efficiency at large energies has been manually added in order to get a better agreement with experimental results. Furthermore, the light-quark jet rejection has been measured for two different $\eta$ regions, which we adapt in our parametrisations. We also perform a reduction in the light-quark tagging rates $(20 \%)$ in order to better agree with experimental cutflows.

Since the $p_{T}$ dependent distributions are given for a particular working point $\bar{\epsilon}_{\mathrm{S}}=0.7$, we linearly rescale the functions to the given chosen signal efficiency $\bar{\epsilon}_{\mathrm{S}}$, or the corresponding background efficiency given by 


\begin{tabular}{llllll}
\hline \hline & $a_{i, 0}$ & $a_{i, 1}$ & $a_{i, 2}$ & $b_{i, 1}$ & $b_{i, 2}$ \\
\hline light & $6.77 \cdot 10^{-6}$ & $-9.86 \cdot 10^{-6}$ & $2.79 \cdot 10^{-5}$ & $-1.99 \cdot 10^{2}$ & $2.50 \cdot 10^{2}$ \\
charm & $-2.97 \cdot 10^{-3}$ & $-2.15 \cdot 10^{-6}$ & $1.29 \cdot 10^{-4}$ & $-1.43 \cdot 10^{2}$ & $1.12 \cdot 10^{2}$ \\
\hline \hline
\end{tabular}

Table 10: The coefficients for the light- and charm-jet efficiency as a function of $\eta$, Eq. 22.

the ROC curves $\left(p_{T}\right.$ in $\left.\mathrm{GeV}\right)$ :

$$
\begin{aligned}
& \epsilon_{\mathrm{S}}\left(p_{\mathrm{T}}\right)=\frac{\bar{\epsilon}_{\mathrm{S}} \cdot 0.85}{0.7}\left(0.552+\frac{0.210}{1+\mathrm{e}^{-0.123 \cdot\left(p_{\mathrm{T}}-47.6\right)}}\right) \\
& \times \frac{0.7+0.05 \cdot \mathrm{e}^{-\frac{p_{\mathrm{T}}}{308}}}{0.75} \begin{cases}1 & \text { if } p_{\mathrm{T}} \leq 100, \\
1-7 \times 10^{-4} \cdot\left(p_{\mathrm{T}}-100\right) & \text { if } p_{\mathrm{T}}>100,\end{cases} \\
& \epsilon_{\text {light }}\left(p_{\mathrm{T}}, \eta\right)=\frac{\bar{r}_{\text {light }}(0.7) \times 0.8}{\bar{r}_{\text {light }}\left(\bar{\epsilon}_{\mathrm{S}}\right)} \begin{cases}1.06 \times 10^{-2}+6.47 \times 10^{-6} \cdot p_{\mathrm{T}}^{2}+4.03 \times 10^{-8} \cdot p_{\mathrm{T}}^{4} & \text { if }|\eta|<1.3, \\
6.61 \times 10^{-3}+6.49 \times 10^{-5} \cdot p_{\mathrm{T}}^{2}-3.12 \times 10^{-8} \cdot p_{\mathrm{T}}^{4} & \text { if } 1.3 \leq|\eta|<2.5,\end{cases} \\
& \epsilon_{\mathrm{c}}\left(p_{\mathrm{T}}\right)=\frac{\bar{r}_{\mathrm{c}}(0.7) \times 0.4}{\bar{r}_{\mathrm{c}}\left(\bar{\epsilon}_{\mathrm{S}}\right)} \frac{0.461}{1+\mathrm{e}^{-0.0464 \cdot\left(p_{\mathrm{T}}-20.4\right)}} .
\end{aligned}
$$

C.3.2. $13 \mathrm{TeV}$

For the ATLAS analyses performed in Run-2, CheckMATE uses efficiencies fitted to the MV2c20 algorithm described in 128. The dependence of the $b$-jet efficiency on $p_{T}$ is kept the same as in Run-1, but the mistag rates differ from their Run-1 equivalents.

The light- and charm-jet efficiencies in CheckMATE depend on both $\eta$ and $p_{T}$, with the following functional dependencies. The dependence on the pseudorapidity $\eta$ is as follows:

$$
\epsilon_{i}\left(\eta, \bar{\epsilon}_{S}\right)=\left(a_{i, 0}+a_{i, 1} \eta+a_{i, 2} \eta^{2}\right) \cdot\left(1+b_{i, 1} \bar{\epsilon}_{S}+b_{i, 2} \bar{\epsilon}_{S}^{2}\right) \cdot \frac{\bar{r}_{i}(0.7)}{\bar{r}_{i}\left(\bar{\epsilon}_{S}\right)},
$$

where $i$ is either $c$ or $l$ for charm and light jets, respectively. The coefficients of these efficiency functions are shown in Table 10 and the functional dependence on the $b$-efficiency is included through the ROC curves $\bar{r}_{i}$, given as

$$
\begin{aligned}
\log _{10}\left[\bar{r}_{\text {light }}\left(\bar{\epsilon}_{S}\right)\right] & =-21.9 \bar{\epsilon}_{S}^{2}+14.5 \bar{\epsilon}_{S}+7.02, \\
\log _{10}\left[\bar{r}_{c}\left(\bar{\epsilon}_{S}\right)\right] & =7.39 \bar{\epsilon}_{S}^{2}-19.7 \bar{\epsilon}_{S}+12.4 .
\end{aligned}
$$

The fitted functions given by Eq. 22 are shown in Figures $5 \mathrm{~b}$ and $6 \mathrm{~b}$ as solid lines, compared to the values from ATLAS, shown as dots.

The $p_{T}$ dependence is given by a piecewise function, where each piece depends on the $p_{T}$ of the jet and on the $b$-efficiency. The form of the pieces is

$$
\epsilon_{i, \alpha}\left(p_{T}, \bar{\epsilon}_{S}\right)=\left[\left(a_{i, \alpha}+b_{i, \alpha} \bar{\epsilon}_{S}+c_{i, \alpha} \bar{\epsilon}_{S}^{2}\right)+\left(d_{i, \alpha}+e_{i, \alpha} \bar{\epsilon}_{S}+f_{i, \alpha} \bar{\epsilon}_{S}^{2}\right) p_{T}\right] \cdot \frac{\epsilon_{s, i}\left(\bar{\epsilon}_{S}\right)}{\epsilon_{s, i}(0.7)},
$$

where $i$ is either $c$ or $l$ for charm and light jets, respectively, and $\alpha$ enumerates the pieces of the function. The coefficients for light and charm jets are given in Tables 11 and 12 .

Outside of the range, $\bar{\epsilon}_{S} \in[0.6,0.85]$, the parameters are frozen to either $60 \%$ or $85 \%$ and the scaling is exclusively given by the scale functions $\bar{r}_{i}$ given by Eqs. (23) and 24. The fitted functions are shown in 


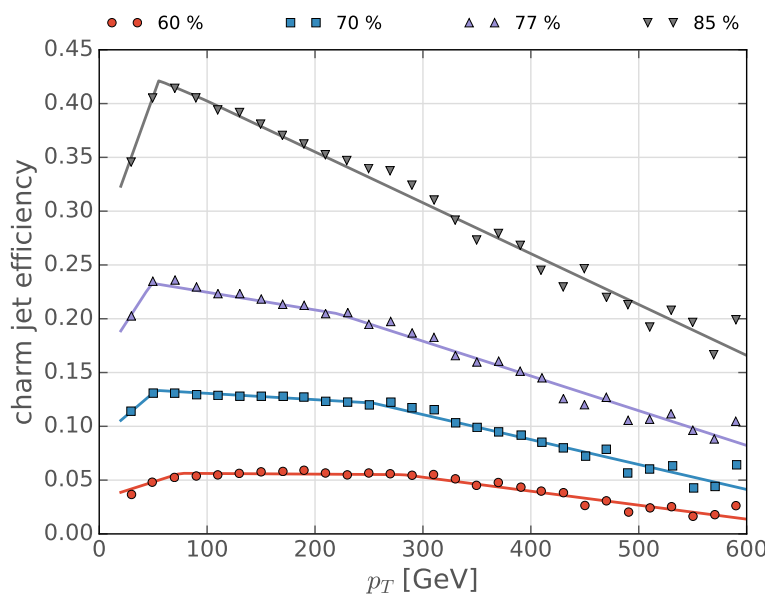

(a) Efficiency vs. $p_{T}$.

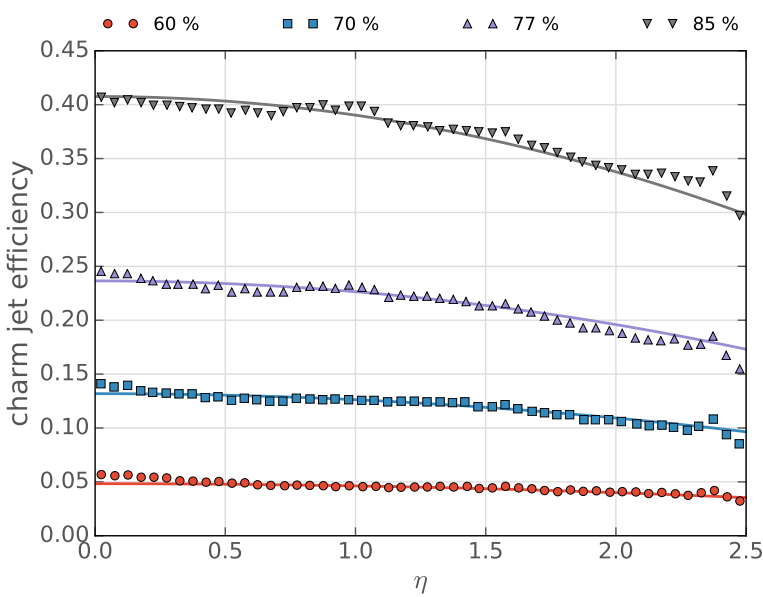

(b) Efficiency vs. $\eta$.

Figure 5: The charm-jet efficiency for the $13 \mathrm{TeV}$ ATLAS $b$-tagger, for four different working points. The points show the expected efficiencies as determined by the ATLAS collaboration. The solid lines are the functions that CheckMATE 2 uses to model the efficiencies.

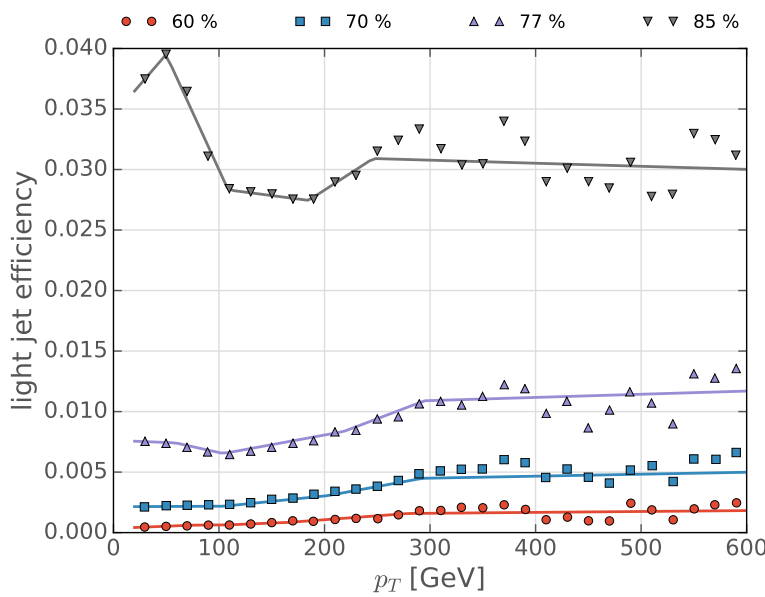

(a) Efficiency vs. $p_{T}$.

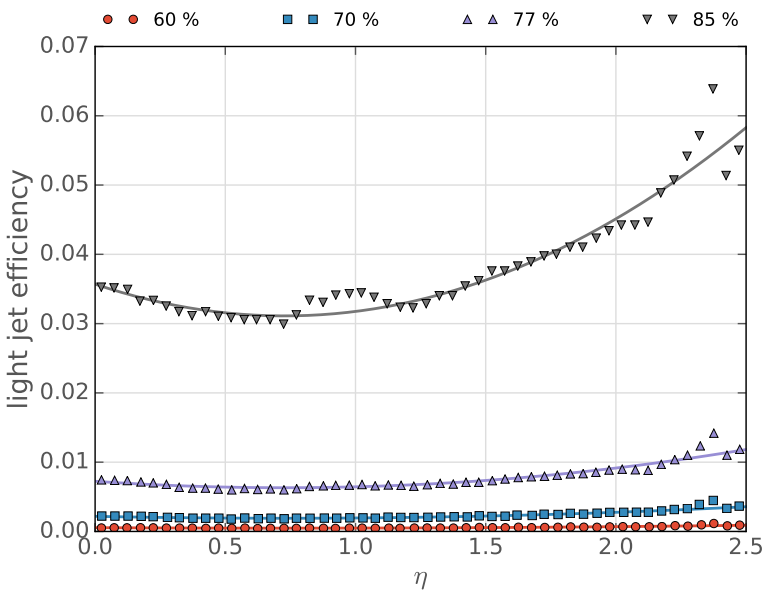

(b) Efficiency vs. $\eta$.

Figure 6: The light-jet efficiency for the $13 \mathrm{TeV}$ ATLAS $b$-tagger, for four different working points. The points show the expected efficiencies as determined by the ATLAS collaboration. The solid lines are the functions that CheckMATE 2 uses to model the efficiencies.

Figures 5 a and $6 \mathrm{a}$ as solid lines, compared to the points they were fitted to. The efficiency functions of $p_{T}$ and $\eta$ are combined into a single function of $p_{T}$ and $\eta$, and normalised using 12 million $t \bar{t}$ events,

$$
\begin{aligned}
\epsilon_{\text {light }}\left(p_{T}, \eta, \bar{\epsilon}_{S}\right) & =\epsilon_{\text {light }}\left(p_{T}, \bar{\epsilon}_{S}\right) \cdot \epsilon_{\text {light }}\left(\eta, \bar{\epsilon}_{S}\right) \cdot 450 \frac{\bar{r}_{\text {light }}\left(\bar{\epsilon}_{S}\right)}{\bar{r}_{\text {light }}(0.7)}\left(1+\frac{3\left(100 \bar{\epsilon}_{S}-60\right)}{1000}\right), \\
\epsilon_{c}\left(p_{T}, \eta, \bar{\epsilon}_{S}\right) & =\epsilon_{c}\left(p_{T}, \bar{\epsilon}_{S}\right) \cdot \epsilon_{c}\left(\eta, \bar{\epsilon}_{S}\right) \cdot 7.5 \frac{\bar{r}_{c}\left(\bar{\epsilon}_{S}\right)}{\bar{r}_{c}(0.7)}\left(1+\frac{100 \bar{\epsilon}_{S}-60}{130}\right) .
\end{aligned}
$$

These efficiencies were tested against the subset of the $13 \mathrm{TeV}$ analyses implemented in CheckMATE 2 that use $b$-jets. The validation for this class of analyses was performed using the $b$-tagger described here and the results were found to be in a good agreement with the published results. 


\begin{tabular}{lcrrrr}
\hline \hline$\alpha$ & \multicolumn{1}{c}{ 1 } & \multicolumn{1}{c}{3} & \multicolumn{1}{l}{4} & \multicolumn{1}{c}{5} \\
\hline$a_{\text {light }, \alpha}$ & -0.0152 & $2.98 \cdot 10^{-3}$ & $2.40 \cdot 10^{-3}$ & $2.40 \cdot 10^{-3}$ & -0.0153 \\
$b_{\text {light }, \alpha}$ & 0.0228 & $-2.57 \cdot 10^{-3}$ & $-4.31 \cdot 10^{-4}$ & $-4.31 \cdot 10^{-3}$ & 0.0147 \\
$c_{\text {light }, \alpha}$ & -0.00637 & $2.70 \cdot 10^{-3}$ & 0 & 0 & 0 \\
$d_{\text {light }, \alpha}$ & 0.000384 & $2.26 \cdot 10^{-5}$ & $3.53 \cdot 10^{-5}$ & $1.13 \cdot 10^{-4}$ & $1.43 \cdot 10^{-5}$ \\
$e_{\text {light }, \alpha}$ & -0.00103 & $-1.64 \cdot 10^{-5}$ & $-1.63 \cdot 10^{-5}$ & $-1.82 \cdot 10^{-4}$ & $-2.32 \cdot 10^{-5}$ \\
$f_{\text {light }, \alpha}$ & 0.000684 & $-2.63 \cdot 10^{-5}$ & $-3.04 \cdot 10^{-5}$ & $6.20 \cdot 10^{-5}$ & $0.74 \cdot 10^{-5}$ \\
\hline \hline
\end{tabular}

Table 11: Coefficients for the $p_{T}$-dependent light-jet efficiency function, Eq. 25.

\begin{tabular}{lccc}
\hline \hline$\alpha$ & 1 & 2 & 3 \\
\hline$a_{c, \alpha}$ & -0.288 & 0.576 & 1.51 \\
$b_{c, \alpha}$ & 0.375 & -0.941 & -2.67 \\
$c_{c, \alpha}$ & -0.0329 & 0.513 & 1.31 \\
$d_{c, \alpha}$ & $1.02 \cdot 10^{-3}$ & $2.13 \cdot 10^{-4}$ & $-2.13 \cdot 10^{-3}$ \\
$e_{c, \alpha}$ & $-2.09 \cdot 10^{-4}$ & $-3.52 \cdot 10^{-4}$ & $4.43 \cdot 10^{-3}$ \\
$f_{c, \alpha}$ & $-5.00 \cdot 10^{-5}$ & $-5.20 \cdot 10^{-5}$ & $-2.45 \cdot 10^{-3}$ \\
\hline \hline
\end{tabular}

Table 12: Coefficients for the $p_{T}$-dependent charm-jet efficiency function, Eq. 25.

\section{References}

[1] J. de Favereau, C. Delaere, P. Demin, A. Giammanco, V. Lemaître, A. Mertens, M. Selvaggi, DELPHES 3, A modular framework for fast simulation of a generic collider experiment, JHEP 02 (2014) 057. arXiv: 1307.6346

[2] M. Cacciari, G. P. Salam, G. Soyez, FastJet User Manual, Eur. Phys. J. C72 (2012) 1896. arXiv:1111.6097

[3] M. Cacciari, G. P. Salam, Dispelling the $N^{3}$ myth for the $k_{t}$ jet-finder, Phys. Lett. B641 (2006) 57-61. arXiv:hep-ph/ 0512210

[4] M. Cacciari, G. P. Salam, G. Soyez, The anti- $k_{t}$ jet clustering algorithm, JHEP 0804 (2008) 063. arXiv:0802.1189

[5] A. L. Read, Presentation of search results: The $C L_{S}$ technique, J. Phys. G28 (2002) 2693-2704.

[6] J. Alwall, R. Frederix, S. Frixione, V. Hirschi, F. Maltoni, O. Mattelaer, H. S. Shao, T. Stelzer, P. Torrielli, M. Zaro, The automated computation of tree-level and next-to-leading order differential cross sections, and their matching to parton shower simulations, JHEP 07 (2014) 079. arXiv:1405.0301

[7] T. Sjöstrand, S. Ask, J. R. Christiansen, R. Corke, N. Desai, P. Ilten, S. Mrenna, S. Prestel, C. O. Rasmussen, P. Z. Skands, An Introduction to PYTHIA 8.2, Comput. Phys. Commun. 191 (2015) 159-177. arXiv:1410.3012

[8] C. Lester, D. Summers, Measuring masses of semiinvisibly decaying particles pair produced at hadron colliders, Phys. Lett. B463 (1999) 99-103. arXiv:hep-ph/9906349

[9] A. Barr, C. Lester, P. Stephens, $m_{T 2}$ : The Truth behind the glamour, J. Phys. G29 (2003) 2343-2363. arXiv:hep-ph/ 0304226

[10] H.-C. Cheng, Z. Han, Minimal Kinematic Constraints and $m_{T 2}$, JHEP 0812 (2008) 063. arXiv:0810.5178

[11] Y. Bai, H.-C. Cheng, J. Gallicchio, J. Gu, Stop the Top Background of the Stop Search, JHEP 1207 (2012) 110. arXiv: 1203.4813

[12] D. R. Tovey, On measuring the masses of pair-produced semi-invisibly decaying particles at hadron colliders, JHEP 0804 (2008) 034. arXiv:0802.2879

[13] G. Polesello, D. R. Tovey, Supersymmetric particle mass measurement with the boost-corrected contransverse mass, JHEP 1003 (2010) 030. arXiv:0910.0174

[14] K. T. Matchev, M. Park, A General method for determining the masses of semi-invisibly decaying particles at hadron colliders, Phys. Rev. Lett. 107 (2011) 061801. arXiv:0910.1584

[15] M. L. Graesser, J. Shelton, Hunting Mixed Top Squark Decays, Phys. Rev. Lett. 111 (12) (2013) 121802. arXiv:1212.4495

[16] M. R. Buckley, J. D. Lykken, C. Rogan, M. Spiropulu, Super-Razor and Searches for Sleptons and Charginos at the LHC, Phys. Rev. D89 (5) (2014) 055020. arXiv:1310.4827 
[17] G. Aad, et al., Combined Measurement of the Higgs Boson Mass in $p p$ Collisions at $\sqrt{s}=7$ and 8 TeV with the ATLAS and CMS Experiments, Phys. Rev. Lett. 114 (2015) 191803. arXiv:1503.07589

[18] G. Aad, et al., Measurements of the Higgs boson production and decay rates and constraints on its couplings from a combined ATLAS and CMS analysis of the LHC $p p$ collision data at $\sqrt{s}=7$ and 8 TeV, JHEP 08 (2016) 045 . arXiv:1606.02266

[19] D. Alves, Simplified Models for LHC New Physics Searches, J. Phys. G39 (2012) 105005. arXiv:1105.2838

[20] S. Kraml, S. Kulkarni, U. Laa, A. Lessa, W. Magerl, D. Proschofsky-Spindler, W. Waltenberger, SModelS: a tool for interpreting simplified-model results from the LHC and its application to supersymmetry, Eur. Phys. J. C74 (2014) 2868. arXiv: 1312.4175

[21] S. Kraml, S. Kulkarni, U. Laa, A. Lessa, V. Magerl, W. Magerl, D. Proschofsky-Spindler, M. Traub, W. Waltenberger, SModelS v1.0: a short user guide. arXiv:1412.1745

[22] M. Papucci, K. Sakurai, A. Weiler, L. Zeune, Fastlim: a fast LHC limit calculator, Eur. Phys. J. C74 (11) (2014) 3163. arXiv: 1402.0492

[23] D. Barducci, A. Belyaev, M. Buchkremer, J. Marrouche, S. Moretti, L. Panizzi, XQCAT: eXtra Quark Combined Analysis Tool, Comput. Phys. Commun. 197 (2015) 263-275. arXiv:1409.3116

[24] S. Caron, J. S. Kim, K. Rolbiecki, R. Ruiz de Austri, B. Stienen, The BSM-AI project: SUSY-AI - Generalizing LHC limits on Supersymmetry with Machine Learning. arXiv:1605.02797

[25] K. Cranmer, I. Yavin, RECAST: Extending the Impact of Existing Analyses, JHEP 04 (2011) 038. arXiv:1010.2506.

[26] M. Drees, H. Dreiner, D. Schmeier, J. Tattersall, J. S. Kim, CheckMATE: Confronting your Favourite New Physics Model with LHC Data, Comput. Phys. Commun. 187 (2015) 227-265. arXiv:1312.2591

[27] J. S. Kim, D. Schmeier, J. Tattersall, K. Rolbiecki, A framework to create customised LHC analyses within CheckMATE, Comput. Phys. Commun. 196 (2015) 535-562. arXiv:1503.01123

[28] E. Conte, B. Fuks, G. Serret, MadAnalysis 5, A User-Friendly Framework for Collider Phenomenology, Comput. Phys. Commun. 184 (2013) 222-256. arXiv:1206.1599

[29] B. Dumont, B. Fuks, S. Kraml, S. Bein, G. Chalons, E. Conte, S. Kulkarni, D. Sengupta, C. Wymant, Toward a public analysis database for LHC new physics searches using MADANALYSIS 5, Eur. Phys. J. C75 (2) (2015) 56. arXiv: 1407.3278

[30] J. M. Butterworth, D. Grellscheid, M. Krämer, D. Yallup, Constraining new physics with collider measurements of Standard Model signatures. arXiv:1606.05296

[31] A. Buckley, J. Butterworth, L. Lonnblad, D. Grellscheid, H. Hoeth, J. Monk, H. Schulz, F. Siegert, Rivet user manual, Comput. Phys. Commun. 184 (2013) 2803-2819. arXiv:1003.0694.

[32] P. Z. Skands, et al., SUSY Les Houches accord: Interfacing SUSY spectrum calculators, decay packages, and event generators, JHEP 07 (2004) 036. arXiv:hep-ph/0311123

[33] B. C. Allanach, et al., SUSY Les Houches Accord 2, Comput. Phys. Commun. 180 (2009) 8-25. arXiv:0801.0045.

[34] C. Degrande, C. Duhr, B. Fuks, D. Grellscheid, O. Mattelaer, T. Reiter, UFO - The Universal FeynRules Output, Comput. Phys. Commun. 183 (2012) 1201-1214. arXiv:1108.2040

[35] N. D. Christensen, C. Duhr, FeynRules - Feynman rules made easy, Comput. Phys. Commun. 180 (2009) $1614-1641$. arXiv:0806.4194

[36] A. Alloul, N. D. Christensen, C. Degrande, C. Duhr, B. Fuks, FeynRules 2.0 - A complete toolbox for tree-level phenomenology, Comput. Phys. Commun. 185 (2014) 2250-2300. arXiv:1310.1921

[37] F. Staub, Exploring new models in all detail with SARAH, Adv. High Energy Phys. 2015 (2015) 840780. arXiv: 1503.04200

[38] F. Staub, SARAH 4 : A tool for (not only SUSY) model builders, Comput. Phys. Commun. 185 (2014) 1773-1790. arXiv:1309.7223.

[39] A. Semenov, LanHEP — A package for automatic generation of Feynman rules from the Lagrangian. Version 3.2, Comput. Phys. Commun. 201 (2016) 167-170. arXiv:1412.5016

[40] Wikipedia, Fritz (chess). [Online; accessed May 20, 2016]. URL https://en.wikipedia.org/wiki/Fritz_(chess)

[41] C. GmbH, ChessBase, [Online; accessed May 20, 2016]. URL http://en.chessbase.com/

[42] W. Beenakker, R. Hopker, M. Spira, P. M. Zerwas, Squark and gluino production at hadron colliders, Nucl. Phys. B492 (1997) 51-103. arXiv:hep-ph/9610490

[43] W. Beenakker, M. Krämer, T. Plehn, M. Spira, P. M. Zerwas, Stop production at hadron colliders, Nucl. Phys. B515 (1998) 3-14. arXiv:hep-ph/9710451

[44] W. Beenakker, M. Klasen, M. Krämer, T. Plehn, M. Spira, P. M. Zerwas, The Production of charginos/neutralinos and sleptons at hadron colliders, Phys. Rev. Lett. 83 (1999) 3780-3783, [Erratum: Phys. Rev. Lett. 100, 029901 (2008)]. arXiv:hep-ph/9906298

[45] M. Spira, Higgs and SUSY particle production at hadron colliders, in: Supersymmetry and unification of fundamental interactions. Proceedings, 10th International Conference, SUSY'02, Hamburg, Germany, June 17-23, 2002, pp. 217-226. arXiv:hep-ph/0211145

[46] T. Plehn, Measuring the MSSM Lagrangean, Czech. J. Phys. 55 (2005) B213-B220. arXiv:hep-ph/0410063

[47] A. Kulesza, L. Motyka, Threshold resummation for squark-antisquark and gluino-pair production at the LHC, Phys. Rev. Lett. 102 (2009) 111802. arXiv:0807.2405

[48] A. Kulesza, L. Motyka, Soft gluon resummation for the production of gluino-gluino and squark-antisquark pairs at the LHC, Phys. Rev. D80 (2009) 095004. arXiv:0905.4749. 
[49] W. Beenakker, S. Brensing, M. Krämer, A. Kulesza, E. Laenen, et al., Soft-gluon resummation for squark and gluino hadroproduction, JHEP 0912 (2009) 041. arXiv:0909.4418

[50] W. Beenakker, S. Brensing, M. Krämer, A. Kulesza, E. Laenen, et al., Supersymmetric top and bottom squark production at hadron colliders, JHEP 1008 (2010) 098. arXiv:1006.4771

[51] W. Beenakker, S. Brensing, M. Krämer, A. Kulesza, E. Laenen, et al., Squark and Gluino Hadroproduction, Int. J. Mod. Phys. A26 (2011) 2637-2664. arXiv:1105.1110.

[52] T. Sjöstrand, S. Mrenna, P. Z. Skands, A Brief Introduction to PYTHIA 8.1, Comput. Phys. Commun. 178 (2008) 852-867. arXiv:0710.3820

[53] The Pythia 8 Collaboration, PYTHIA 8 Online Documentation [Online; accessed May 12, 2016]. URL http://home.thep.lu.se/ torbjorn/pythia82html/Welcome.html

[54] W. Kilian, T. Ohl, J. Reuter, WHIZARD: Simulating Multi-Particle Processes at LHC and ILC, Eur. Phys. J. C71 (2011) 1742. arXiv:0708.4233.

[55] M. Moretti, T. Ohl, J. Reuter, O'Mega: An Optimizing matrix element generator. arXiv:hep-ph/0102195

[56] A. Belyaev, N. D. Christensen, A. Pukhov, CalcHEP 3.4 for collider physics within and beyond the Standard Model, Comput. Phys. Commun. 184 (2013) 1729-1769. arXiv:1207.6082.

[57] Mini-workshop on recasting ATLAS and CMS new physics searches. URL https://lpsc-indico.in2p3.fr/Indico/event/1085/

[58] K. A. Olive, et al., Review of Particle Physics, Chin. Phys. C38 (2014) 090001.

[59] J. S. Kim, K. Rolbiecki, K. Sakurai, J. Tattersall, 'Stop' that ambulance! New physics at the LHC?, JHEP 12 (2014) 010. arXiv: 1406.0858

[60] R. D. Ball, et al., Parton distributions with LHC data, Nucl. Phys. B867 (2013) 244-289. arXiv:1207.1303

[61] G. Aad, et al., Search for squarks and gluinos with the ATLAS detector in final states with jets and missing transverse momentum using $\sqrt{s}=8 \mathrm{TeV}$ proton-proton collision data, JHEP 09 (2014) 176. arXiv:1405.7875

[62] G. Aad, et al., Search for new phenomena in final states with an energetic jet and large missing transverse momentum in pp collisions at $\sqrt{s}=8 \mathrm{TeV}$ with the ATLAS detector, Eur. Phys. J. C75 (7) (2015) 299, [Erratum: Eur. Phys. J. C75, no. 9, 408 (2015)]. arXiv:1502.01518

[63] S. Chatrchyan, et al., Search for supersymmetry in hadronic final states with missing transverse energy using the variables $\alpha_{T}$ and b-quark multiplicity in pp collisions at $\sqrt{s}=8 \mathrm{TeV}$, Eur. Phys. J. C73 (9) (2013) 2568. arXiv:1303.2985.

[64] G. Aad, et al., Search for new phenomena in final states with large jet multiplicities and missing transverse momentum at $\sqrt{s}=8 \mathrm{TeV}$ proton-proton collisions using the ATLAS experiment, JHEP 10 (2013) 130, [Erratum: JHEP 01 (2014) 109]. $\operatorname{arXiv:1308.1841}$

[65] J. Cao, L. Shang, J. M. Yang, Y. Zhang, Explanation of the ATLAS Z-Peaked Excess in the NMSSM, JHEP 06 (2015) 152. arXiv:1504.07869.

[66] G. Aad, et al., Search for direct third-generation squark pair production in final states with missing transverse momentum and two $b$-jets in $\sqrt{s}=8 \mathrm{TeV} p p$ collisions with the ATLAS detector, JHEP 1310 (2013) 189. arXiv:1308.2631

[67] G. Aad, et al., Search for direct production of charginos and neutralinos in events with three leptons and missing transverse momentum in $\sqrt{s}=8 \mathrm{TeV} p p$ collisions with the ATLAS detector, JHEP 1404 (2014) 169. arXiv:1402.7029

[68] G. Aad, et al., Search for direct top-squark pair production in final states with two leptons in $p p$ collisions at $\sqrt{ } s=8$ TeV with the ATLAS detector, JHEP 06 (2014) 124. arXiv:1403.4853

[69] G. Aad, et al., Search for direct top squark pair production in events with a $Z$ boson, $b$-jets and missing transverse momentum in $\sqrt{s}=8 \mathrm{TeV} p p$ collisions with the ATLAS detector, Eur. Phys. J. C74 (6) (2014) 2883. arXiv:1403.5222

[70] G. Aad, et al., Search for supersymmetry at $\sqrt{s}=8 \mathrm{TeV}$ in final states with jets and two same-sign leptons or three leptons with the ATLAS detector, JHEP 06 (2014) 035. arXiv:1404.2500

[71] G. Aad, et al., Search for top squark pair production in final states with one isolated lepton, jets, and missing transverse momentum in $\sqrt{s}=8 \mathrm{TeV} p p$ collisions with the ATLAS detector, JHEP 1411 (2014) 118. arXiv:1407.0583

[72] G. Aad, et al., Search for pair-produced third-generation squarks decaying via charm quarks or in compressed supersymmetric scenarios in $p p$ collisions at $\sqrt{s}=8 \mathrm{TeV}$ with the ATLAS detector, Phys. Rev. D90 (5) (2014) 052008. arXiv: 1407.0608

[73] G. Aad, et al., Search for new phenomena in events with a photon and missing transverse momentum in $p p$ collisions at $\sqrt{s}=8 \mathrm{TeV}$ with the ATLAS detector, Phys. Rev. D91 (1) (2015) 012008, [Erratum: Phys. Rev. D92, no. 5, 059903 (2015)]. arXiv:1411.1559

[74] G. Aad, et al., Search for direct pair production of a chargino and a neutralino decaying to the 125 GeV Higgs boson in $\sqrt{s}=8 \mathrm{TeV} p p$ collisions with the ATLAS detector, Eur. Phys. J. C75 (5) (2015) 208. arXiv:1501.07110

[75] G. Aad, et al., Search for supersymmetry in events containing a same-flavour opposite-sign dilepton pair, jets, and large missing transverse momentum in $\sqrt{s}=8 \mathrm{TeV} p p$ collisions with the ATLAS detector, Eur. Phys. J. C75 (7) (2015) 318. arXiv: 1503.03290

[76] G. Aad, et al., ATLAS Run 1 searches for direct pair production of third-generation squarks at the Large Hadron Collider, Eur. Phys. J. C75 (10) (2015) 510, [Erratum: Eur. Phys. J. C76, no. 3, 153 (2016)]. arXiv: 1506.08616

[77] Search for supersymmetry at $\sqrt{s}=8 \mathrm{TeV}$ in final states with jets, missing transverse momentum and one isolated lepton, Tech. Rep. ATLAS-CONF-2012-104, CERN, Geneva (Aug 2012).

[78] Search for New Phenomena in Monojet plus Missing Transverse Momentum Final States using $10 \mathrm{fb}^{-1} \mathrm{of} p \mathrm{Collisions}$ at $\sqrt{s}=8 \mathrm{TeV}$ with the ATLAS detector at the LHC, Tech. Rep. ATLAS-CONF-2012-147, CERN, Geneva (Nov 2012).

[79] Search for direct production of the top squark in the all-hadronic $t \bar{t}+E_{T}^{\text {miss }}$ final state in 21 fb ${ }^{-1}$ of $p p$ collisions at $\sqrt{s}=8 \mathrm{TeV}$ with the ATLAS detector, Tech. Rep. ATLAS-CONF-2013-024, CERN, Geneva (Mar 2013).

[80] Search for direct-slepton and direct-chargino production in final states with two opposite-sign leptons, missing transverse 
momentum and no jets in $20 / \mathrm{fb}$ of $p p$ collisions at $\sqrt{s}=8 \mathrm{TeV}$ with the ATLAS detector, Tech. Rep. ATLAS-CONF2013-049, CERN, Geneva (May 2013).

[81] Search for strong production of supersymmetric particles in final states with missing transverse momentum and at least three $b$-jets using $20.1 \mathrm{fb}^{-1}$ of $p p$ collisions at $\sqrt{s}=8 \mathrm{TeV}$ with the ATLAS Detector, Tech. Rep. ATLAS-CONF-2013-061, CERN, Geneva (Jun 2013).

[82] The ATLAS Collaboration, Search for strongly produced supersymmetric particles in decays with two leptons at $\sqrt{s}=$ $8 \mathrm{TeV}$, Tech. Rep. ATLAS-CONF-2013-089, CERN, Geneva (Aug 2013).

[83] The ATLAS Collaboration, Search for an Invisibly Decaying Higgs Boson Produced via Vector Boson Fusion in $p p$ Collisions at $\sqrt{s}=8 \mathrm{TeV}$ using the ATLAS Detector at the LHC, Tech. Rep. ATLAS-CONF-2015-004, CERN, Geneva (Mar 2015).

[84] V. Khachatryan, et al., Search for dark matter, extra dimensions, and unparticles in monojet events in proton-proton collisions at $\sqrt{s}=8 \mathrm{TeV}$, Eur. Phys. J. C75 (5) (2015) 235. arXiv:1408.3583

[85] V. Khachatryan, et al., Search for Physics Beyond the Standard Model in Events with Two Leptons, Jets, and Missing Transverse Momentum in $p p$ Collisions at $\sqrt{s}=8 \mathrm{TeV}$, JHEP 04 (2015) 124. arXiv:1502.06031.

[86] V. Khachatryan, et al., Search for the production of dark matter in association with top-quark pairs in the single-lepton final state in proton-proton collisions at $\sqrt{s}=8 \mathrm{TeV}$, JHEP 06 (2015) 121. arXiv:1504.03198.

[87] S. Baek, P. Ko, P. Wu, Top-philic Scalar Dark Matter with a Vector-like Fermionic Top Partner, JHEP 10 (2016) 117. arXiv: 1606.00072

[88] The CMS Collaboration, Search for new physics in events with same-sign dileptons and jets in $p p$ collisions at 8 TeV, Tech. Rep. CMS-PAS-SUS-13-013 (2013).

[89] G. Aad, et al., Search for supersymmetry at $\sqrt{s}=13 \mathrm{TeV}$ in final states with jets and two same-sign leptons or three leptons with the ATLAS detector, Eur. Phys. J. C76 (5) (2016) 259. arXiv:1602.09058

[90] M. Aaboud, et al., Search for new phenomena in events with a photon and missing transverse momentum in $p p$ collisions at $\sqrt{s}=13 \mathrm{TeV}$ with the ATLAS detector, JHEP 06 (2016) 059. arXiv:1604.01306

[91] M. Aaboud, et al., Search for new phenomena in final states with an energetic jet and large missing transverse momentum in $p p$ collisions at $\sqrt{s}=13 \mathrm{TeV}$ using the ATLAS detector, Phys. Rev. D94 (3) (2016) 032005. arXiv:1604.07773

[92] M. Aaboud, et al., Search for squarks and gluinos in final states with jets and missing transverse momentum at $\sqrt{s}=13$ TeV with the ATLAS detector, Eur. Phys. J. C76 (7) (2016) 392. arXiv:1605.03814

[93] G. Aad, et al., Search for gluinos in events with an isolated lepton, jets and missing transverse momentum at $\sqrt{s}=13$ TeV with the ATLAS detector, Eur. Phys. J. C76 (10) (2016) 565. arXiv:1605.04285

[94] G. Aad, et al., Search for pair production of gluinos decaying via stop and sbottom in events with $b$-jets and large missing transverse momentum in $p p$ collisions at $\sqrt{s}=13 \mathrm{TeV}$ with the ATLAS detector, Phys. Rev. D94 (3) (2016) 032003. arXiv: 1605.09318

[95] M. Aaboud, et al., Search for top squarks in final states with one isolated lepton, jets, and missing transverse momentum in $\sqrt{s}=13 \mathrm{TeV} p p$ collisions with the ATLAS detector, Phys. Rev. D94 (5) (2016) 052009. arXiv:1606.03903

[96] A search for Supersymmetry in events containing a leptonically decaying $Z$ boson, jets and missing transverse momentum in $\sqrt{ } s=13 \mathrm{TeV} p p$ collisions with the ATLAS detector Tech. Rep. ATLAS-CONF-2015-082, CERN, Geneva (Dec 2015). URL http://cds . cern.ch/record/2114854

[97] Search for production of vector-like top quark pairs and of four top quarks in the lepton-plus-jets final state in $p p$ collisions at $\sqrt{s}=13 \mathrm{TeV}$ with the ATLAS detector Tech. Rep. ATLAS-CONF-2016-013, CERN, Geneva (Mar 2016). URL http://cds.cern.ch/record/2140998

[98] Search for top squarks in final states with one isolated lepton, jets, and missing transverse momentum in $\sqrt{s}=13$ TeV $p p$ collisions with the ATLAS detector, Tech. Rep. ATLAS-CONF-2016-050, CERN, Geneva (Aug 2016). URL https://cds.cern.ch/record/2206132

[99] Search for direct top squark pair production and dark matter production in final states with two leptons in $\sqrt{s}=13$ TeV $p p$ collisions using $13.3 \mathrm{fb}^{-1}$ of ATLAS data, Tech. Rep. ATLAS-CONF-2016-076, CERN, Geneva (Aug 2016). URL http://cds.cern.ch/record/2206249

[100] Search for new physics in final states with two opposite-sign same-flavor leptons, jets and missing transverse momentum in $p p$ collisions at $\sqrt{s}=13 \mathrm{TeV}$, Tech. Rep. CMS-PAS-SUS-15-011, CERN, Geneva (2015). URL https://cds.cern.ch/record/2114811

[101] Search for Supersymmetry at the high luminosity LHC with the ATLAS experiment, Tech. Rep. ATL-PHYS-PUB-2014010, CERN, Geneva (Jul 2014). URL http://cds.cern.ch/record/1735031

[102] Prospects for benchmark Supersymmetry searches at the high luminosity LHC with the ATLAS Detector Tech. Rep. ATL-PHYS-PUB-2013-011, CERN, Geneva (Sep 2013). URL http://cds.cern.ch/record/1604505

[103] A. J. Barr, C. G. Lester, A Review of the Mass Measurement Techniques proposed for the Large Hadron Collider, J. Phys. G37 (2010) 123001. arXiv:1004.2732

[104] W. van Neerven, J. Vermaseren, K. Gaemers, Lepton - jet events as a signature for $W$ production in p anti-p collisions, Tech. rep. (1982).

[105] G. Arnison, et al., Experimental Observation of Isolated Large Transverse Energy Electrons with Associated Missing Energy at $\sqrt{s}=540 \mathrm{GeV}$, Phys. Lett. B122 (1983) 103-116.

[106] M. Banner, et al., Observation of Single Isolated Electrons of High Transverse Momentum in Events with Missing Transverse Energy at the CERN anti-p p Collider, Phys. Lett. B122 (1983) 476-485.

[107] J. Smith, W. van Neerven, J. Vermaseren, The Transverse Mass and Width of the W Boson, Phys. Rev. Lett. 50 (1983) 
1738 .

[108] V. D. Barger, T. Han, R. Phillips, Improved Transverse Mass Variable for Detecting Higgs Boson Decays Into $Z$ Pairs, Phys. Rev. D36 (1987) 295.

[109] L. Randall, D. Tucker-Smith, Dijet Searches for Supersymmetry at the LHC, Phys. Rev. Lett. 101 (2008) 221803. arXiv:0806.1049

[110] V. Khachatryan, et al., Search for Supersymmetry in $p p$ Collisions at $7 \mathrm{TeV}$ in Events with Jets and Missing Transverse Energy, Phys. Lett. B698 (2011) 196-218. arXiv:1101.1628

[111] C. Rogan, Kinematical variables towards new dynamics at the LHC arXiv:1006.2727

[112] S. Chatrchyan, et al., Inclusive search for squarks and gluinos in $p p$ collisions at $\sqrt{ } s=7 \mathrm{TeV}$, Phys. Rev. D85 (2012) 012004. arXiv: 1107.1279

[113] V. Khachatryan, et al., Search for Supersymmetry Using Razor Variables in Events with $b$-Tagged Jets in $p p$ Collisions at $\sqrt{s}=8 \mathrm{TeV}$, Phys. Rev. D91 (2015) 052018. arXiv:1502.00300

[114] C. Chen, New approach to identifying boosted hadronically-decaying particle using jet substructure in its center-of-mass frame, Phys. Rev. D85 (2012) 034007. arXiv:1112.2567

[115] R. Brun, F. Rademakers, ROOT: An object oriented data analysis framework, Nucl. Instrum. Meth. A389 (1997) 81-86.

[116] M. Dobbs, J. B. Hansen, The HepMC C++ Monte Carlo event record for High Energy Physics, Comput. Phys. Commun. 134 (2001) 41-46.

[117] K. Cranmer, Practical Statistics for the LHC in: Proceedings, 2011 European School of High-Energy Physics (ESHEP 2011), 2015, pp. 267-308, [247(2015)]. arXiv:1503.07622

URL https://inspirehep.net/record/1356277/files/arXiv:1503.07622.pdf

[118] S. S. Wilks, The large-sample distribution of the likelihood ratio for testing composite hypotheses Ann. Math. Statist. 9 (1) (1938) 60-62.

URL http://dx.doi.org/10.1214/aoms/1177732360

[119] M. Dowell, P. Jarratt, The "Pegasus" method for computing the root of an equation, BIT Numerical Mathematics 12 (4) (1972) 503-508.

URL http://dx.doi .org/10.1007/BF01932959

[120] Electron efficiency measurements with the ATLAS detector using the 2015 LHC proton-proton collision data Tech. Rep. ATLAS-CONF-2016-024, CERN, Geneva (Jun 2016). URL http://cds.cern.ch/record/2157687

[121] Electron identification efficiency measured with $Z \rightarrow e e$ events using 2016 data, [Online; accessed 12-Nov-2016]. URL https://atlas.web.cern.ch/Atlas/GROUPS/PHYSICS/PLOTS/EGAM-2016-002/

[122] Performance assumptions for an upgraded ATLAS detector at a High-Luminosity LHC Tech. Rep. ATL-PHYS-PUB2013-004, CERN, Geneva (Mar 2013). URL https://cds.cern.ch/record/1527529

[123] G. Aad, et al., Muon reconstruction performance of the ATLAS detector in proton-proton collision data at $\sqrt{s}=13 \mathrm{TeV}$, Eur. Phys. J. C76 (5) (2016) 292. arXiv:1603.05598

[124] Performance assumptions based on full simulation for an upgraded ATLAS detector at a High-Luminosity LHC, Tech. Rep. ATL-PHYS-PUB-2013-009, CERN, Geneva (Sep 2013). URL http://cds . cern.ch/record/1604420

[125] Measurement of the $b$-tag Efficiency in a Sample of Jets Containing Muons with $5 \mathrm{fb}^{-1}$ of Data from the ATLAS Detector, Tech. Rep. ATLAS-CONF-2012-043, CERN, Geneva (Mar 2012).

[126] Measuring the $b$-tag efficiency in a top-pair sample with $4.7 \mathrm{fb}^{-1}$ of data from the ATLAS detector, Tech. Rep. ATLASCONF-2012-097, CERN, Geneva (Jul 2012)

[127] Measurement of the Mistag Rate with $5 \mathrm{fb}^{-1}$ of Data Collected by the ATLAS Detector, Tech. Rep. ATLAS-CONF2012-040, CERN, Geneva (Mar 2012).

[128] Expected performance of the ATLAS b-tagging algorithms in Run-2, Tech. Rep. ATL-PHYS-PUB-2015-022, CERN, Geneva (Jul 2015).

URL http://cds . cern. ch/record/2037697 